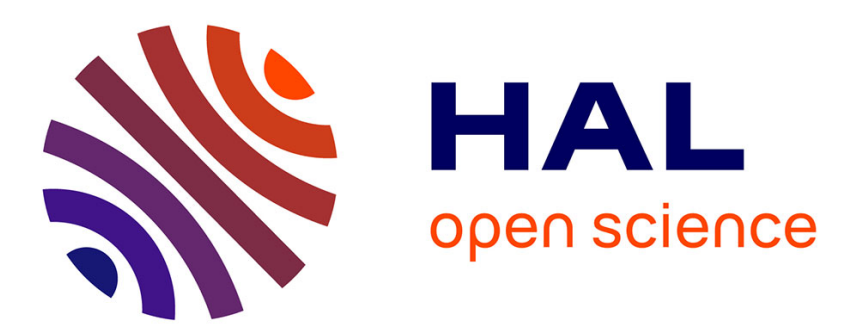

\title{
Etude générale de l'amplification maser par une vapeur alcaline soumise au pompage optique
}

Francis Hartmann

\section{To cite this version:}

Francis Hartmann. Etude générale de l'amplification maser par une vapeur alcaline soumise au pompage optique. Physique Atomique [physics.atom-ph]. Université Pierre et Marie Curie - Paris VI, 1968. Français. NNT: . tel-00011802

\section{HAL Id: tel-00011802 \\ https://theses.hal.science/tel-00011802}

Submitted on 8 Mar 2006

HAL is a multi-disciplinary open access archive for the deposit and dissemination of scientific research documents, whether they are published or not. The documents may come from teaching and research institutions in France or abroad, or from public or private research centers.
L'archive ouverte pluridisciplinaire HAL, est destinée au dépôt et à la diffusion de documents scientifiques de niveau recherche, publiés ou non, émanant des établissements d'enseignement et de recherche français ou étrangers, des laboratoires publics ou privés. 


\section{LABORATOIRE DE PHYSIQUE DE L'ECOLE NORMALE SUPERIEURE}

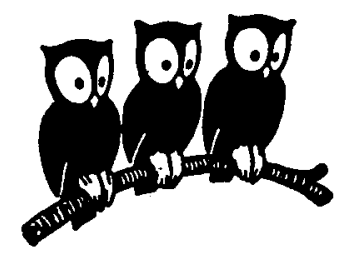

THESE DE DOCTORAT D'ETAT ES SCIENCES PHYSIQUES

présentée

A LA FACULTE DES SCIENCES DE PARIS

par

Francis $\quad H A R T M A N N$

pour abtenir le grade de Docteur ès Sclences

Sujet de la Thèse :

soutenue le
"ETUDE GENERALE DE L'AMPLIFICATION MASER PAR UNE VAPEUR ALCALINE SOUMISE AU POMPAGE OPTIQUE. APPLICATION AU CAS

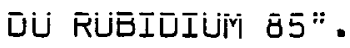

1968 devant la Commission d'Examen

MM. A. KASTLER, Président

$P$. GRIVET

$P$. JACQUINOT

M. ARDITI 
$N^{\circ} d^{\prime}$ enregistrement

au C.N.R.S.

A. 0.2007

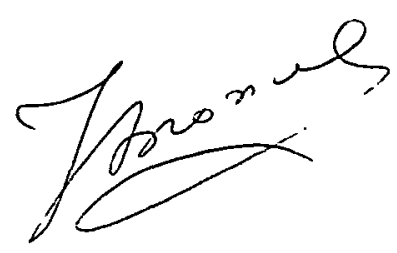

THESE DE DOCTORAT D'ETAT ES SCIENCES PHYSIQUES

présentée

A LA FACULTE DES SCIENCES DE PARIS

par

Francis $\quad H A R T M A N N$

pour obtenir

le grade de Docteur ès Sciences

Sujet de la Thèse : "ETUDE GENERALE DE L'AMPLIFICATION MASER PAR UNE VAPEUR ALCALINE SOUMISE AU POMPAGE OPTIQUE. APPLICATION AU CAS DU RUBIDIUM 85".

soutenue le

1968 devant la Commission d'Examen

$\left.\begin{array}{l}\text { M. A. KASTLER } \\ \text { P. GRIVIET } \\ \text { P. JACQUINOT } \\ \text { M. ARDITI }\end{array}\right\} \quad$ Président

$1968-02$ 
Le présent travail a été effectué au Laboratoire de Physique de I'Ecole Normale Supérieure, sous la direction de Monsieur le Professeur KASTLER que je remercie profondément d'avoir bien voulu m'accueillir dans san équipe, à mon retour du service militaire, en Mars 1961.

Dès le début de ces recherches, commencées en Juillet 1962, Monsieur le Professeur BROSSEL en a suivi l'avancement de près et s'est lui-même chargé de l'exécution de certaines opérations particulièrement délicates, telles que le remplissage des cellules. Je lui en suis très reconnaissant.

J'ai bénéficié de l'excellente ambiance du groupe de Spectroscopie Hertzienne et je suis heureux d'avoir ici l'occasion de remercier tous mes camarades pour les discussions que nous avons eues. Je remercie également les ingénieurs et techniciens du groupe et de l'atelier général du Laboratoire qui m'ont apporté une aide indispensable, notamment Mansieur LEVY, qui a réalisé une partie de la chaîne multiplicatrice de fréquence et Monsieur THOMMÉ, qui a usiné de nombreuses pièces mécaniques. Après avoir assumé la lourde charge administrative des nombreuses commandes passées aux fournisseurs, Mademoiselle BRODSCHI a effectué la frappe du manuscrit avec sa gentillesse, sa rapidité et sa compétence habituelles; je lui exprime ici toute ma gratitude.

Je suis profondément reconnaissant à ma femme de son aide matérielle et morale qui m'a été fort précieuse pendant ces années de travail parfois difficile.

Lors de la construction du montage expérimental, j'ai eu à résoudre de nombreux problèmes technologiques. Ceci m'a conduit à rechercher des conseils ou une participation matérielle auprès de plusieurs établissements et firmes spécialisés en électronique ou en technique du vide, où j'ai toujours trouvé un excellent accueil. Je remercie vivement en particulier Messieurs BECQUEMONT et GRILLOT, du Laboratoire Central des Télécommunications, pour l'aide répétée qu'ils m'ant apportée lors de la mise au point des cavités, et Messieurs COMBET et DELIGNE, du Centre National d'Etudes des Télécommunications, qui m'ont très aimablement prêté un amplificateur à diode tunnel.

Pendant toute cette période, j'ai bénéficié d'une allocation d'attaché de recherche au Centre National de la Recherche Scientifique, le montage expérimental étant réalisé grâce au concours financier de la Direction des Recherches et Moyens d'Essais.

J'adresse enfin à Monsieur le Professeur ROCARD, Directeur du Laboratoire, tous mes remerciements pour sa généreuse hospitalité. 
CHAPITRE I : LE SYSTEME ATOMIQUE

CHAPITRE II : METHODES D'OBSERVATION DU SYSTEME ATOMIQUE

A) ETUDE DE L'ABSORPTION LUMINEUSE EN L'ABSENCE DE RADIOFREQUENCE 14

B) DETECTION OPTIQUE DE LA RESONANCE D-0 23

C) MODULATIONS LUMINEUSES AUX FREQUENCES HYPERFINES 24

D) DETECTION RADIOELECTRIQUE DE LA RESONANCE D-0 25

CHAPITRE III : AMPLIFICATION MASER PAR LA TRANSITION 0-0 27

A) DEFINITIONS ET FORMULES GENERALES 27

B) APPLICATIONS $\quad 34$

1) Condition d'oscillation 34

2) Cavité très désaccordée 35

3) Cavité accordée 36

4) Amplitude du champ oscillant $\vec{H} \quad 38$

5) Retour sur la détection optique 40

CHAPITRE IV : ETUDE DE L'OSCILLATION

CHAPITRE $V$ : APPLICATION AU CAS DU RUBIDIUM 85 : TECHNIQUES EXPERIMENTALES 47

$\begin{array}{ll}\text { A) POMPAGE OPTIQUE DU RUBIDIUM } 85 & 47\end{array}$

B) DESCRIPTION DU MONTAGE EXPERIMENTAL 51

1) Ensemble cellule-cavité

2) Partie optique $\quad 52$

3) Générateur hyperfréquence

4) Récepteur hyperfréquence 56

5) Oscillateur à réaction extérieure 58

C) STRUCTURE DE L'ENSEMBLE CELLULE-CAVITE

1) Choix du mode de résonance de la cavité 59

2) Configurations étudiées

3) Technique de remplissage $\quad 65$

CHAPITRE VI : RESULTATS DES MESURES ET INTERPRETATION 66

A) GENERALITES $\quad 66$ 
B) ETUDE DE L'ABSORPTION LUMINEUSE EN L'ABSENCE DE RADIOFREQUENCE

1) Variation de la constante de temps de pompage $\tau_{p}$ avec l'intensité d'illumination

2) Variation du temps de pompage $T_{p}$ avec la température de la cellule

3) Variation du temps de relaxation $T_{1 H}$ avec la température de la cellule

4) Variation de l'amplitude de l'exponentielle de pompage $\tilde{L}_{A}-\hat{L}_{A}$ avec l'intensité d'illumination

5) Variation de l'amplitude de l'exponentielle de pompage $\tilde{L}_{A}-\widehat{L}_{A}$ et de la lumière absorbée $\hat{L}_{A}$ avec la température de la cellule

C) DETECTION OPTIQUE DE LA RESONANCE 0-0 75

1) Déplacement de la résonance 0-0 sous l'effet d'un gaz étranger 76

2) Largeur de la résonance 0-0 77

D) ESSAI D'OBSERVATION DES MODULATIONS LUMINEUSES AUX FREQUENCES HYPERFINES 78

E) AMPLIFICATION MASER PAR LA TRANSITION 0-0 78

1) Influence du champ magnétique statique 79

2) Déplacement de la résanance 0-0 sous l'effet de la lumière 80

3) Rétrécissement de raie par réaction 81

4) Variation de l'amplification avec l'intensité d'illumination 82

5) Variation de l'amplification avec la pression de gaz étranger dans la cellule

6) Variation de l'amplification avec la température de la cellule 83

7) Variation de 1 'amplification avec les caractéristiques du filtre 83

8) Régimes transitoires 86

F) ETUDE DE L'OSCILLATION AVEC REACTION EXTERIEURE

CONCLUSION

APPENDICE I : CARTE DU MODE TE 011

APPENDICE II : MOUVEMENT DES ATOMES ET COEFFICIENT DE REMPLISSAGE

A) FORME GENERALE DES SIGNAUX DE RESONANCE

B) CELLULE SANS GAZ ETRANGER $\quad 98$

C) CELLULE AVEC GAZ ETRANGER 102

APPENDICE III : TECHNOLOGIE DE CONSTRUCTION DE L'ENSEMBLE CELLULE-CAVITE 106

A) CONFIGURATIONS AVEC CELLULE INTERIEURE 107

B) CONFIGURATIONS SANS CELLULE INTERIEURE 109 REFERENCES 
Dans leur état fondamental ${ }^{2} \mathrm{~S}_{1 / 2}$, les atomes alcalins de spin nucléaire demi-entier présentent une résonance dite "D-O" dont la fréquence va s'étage de 254 MHz pour ${ }^{41} \mathrm{~K}$ à 9 193 $\mathrm{MHz}$ pour ${ }^{133} \mathrm{Cs}$. La finesse exceptionnelle de cette résonance $\left(\Delta \nu_{a} / \nu_{a}\right.$ $\approx 10^{8}$ J, liée à sa relative insensibilité aux champs magnétiques, est déjà mise à profit dans plusieurs standards de fréquence : étalons à jet de césium, horloges à vapeur de rubidium ( ${ }^{l}$ ). Le problème est dans tous les cas de construire un oscillateur dont la fréquence soit contrôlée par celle de la transition atomique. Une méthode particulièrement intéressante pour arriver à ce résultat consiste à essayer d'obtenir l'autooscillation maser du système atomique sur la raie correspondante. On sait en effet, depuis longtemps, que certains modes de pompage optique (en particulier celui qui est utilisé dans les horloges à rubldiuml produisent, dans une vapeur alcaline, une inversion de populations entre les sous-niveaux $\mid F_{+}, 0>$ et $\mid F_{-}$, $0>$ bordant la transition $0-0$. Une onde électromagnétique de fréquence $\nu_{a}$, interagissant avec les atomes, est alors susceptible d’être amplifíe : si la vapeur alnsi pompée est enfermée dans une cavité résonnante accordée à cette fréquence et de pertes radioélectriques suffisamment faibles, l'effet de réaction pourra conduire a une autooscillation entretenue. Comme nous le verrons, le calcul lalsse prévalr pour cet osclllateur un niveau de sortie très faible $(P \simeq 10-10$ watt: $)$, une derive de fréquence à long terme comparable à celle des horloges à rubidium $\left(\Delta \nu / \nu \lesssim 3.10^{-11}\right.$ sur un mols), mals une excellente stabilité de fréquence à court terme $(\Delta \nu / \nu<10-12$ sur 0,1 seconde $)$.

Cette dernière caractéristique donne à l'appareil tout son intérêt. En effet, si l'on dispose actuellement de nombreux standards dont la stabilité à long ou moyen terme est très bonne, la situation est beaucoup moins satis$\ldots / \ldots$ 
faisante en ce qui concerne la stabilité à court terme (sur une seconde ou moins); parmi les étalons atomiques notamment, les masers à ammoniac et à hydrogène sont actuellement les seuls à présenter sur ce point des performances intéressantes. Le caractère "actif" d'un autooscillateur maser lui confère en effet des avantages particuliers à cet égard, et le maser à vapeur alcaline, dont le domaine de stabilité maximum se situe, comme nous le verrons, entre ceux des masers à ammoniac et à hydrogène, devrait compléter les propriétés des deux appareils précédents. Sa stabilité et le volume réduit sous lequel on doit pouvoir le réaliser lui ouvrirait d'importantes applications en navigation Doppler et en télémétrie. Dans le domaine de la physique fondamentale, il permettrait une étude systématique et très fine des déplacements de fréquence associés aux différentes perturbations affectant les atomes alcalins en phase vapeur : collisions contre les parois ou contre les molécules d'un gaz étranger, phénomènes d’échange, action d'un champ électrique, etc., ainsi peut-être que la vérification de certains effets relativistes.

Les caractéristiques précédentes ont attiré l'attention très tôt, dès les premières observations de la résonance 0-0 par pompage et détection optiques. Des considérations relatives à l'efficacité du pompage des différents alcalins, sur lesquelles nous aurons l'occasion de revenir, ont conduit plusieurs laboratoires à tenter d'abord l'expérience sur le rubidium 87. Les travaux sur cet isotope $\left(\nu_{a} \simeq 6834,7 \mathrm{MHz}\right), \mathrm{com}-$ mencés en 1956 (2), ont permis d'obtenir I'oscillation en $1964\left({ }^{3}\right)\left({ }^{4}\right)$. Les très grandes difficultés rencontrées ont jusqu'à présent empêché une étude détaillée de cet oscillateur, en particulier de sa stabilité en fréquence.

Nos propres recherches, commencées indépendamment en Juillet 1962, ont porté sur l'isotope 85 du rubidium $\left(\nu_{a} \simeq 3035,7 \mathrm{MHz}\right)$, ce choix étant essentiellement motivé par la possibilité d'un pompage optique dont l'efficacité semblait comparable 
à celle du pompage de ${ }^{87} \mathrm{Rb}$. La mise au point d'enduits paraffinés réduisant d'une façon considérable la relaxation par collisions sur les parois $\left({ }^{5}\right)\left({ }^{6}\right)$ paraissait également favorable à la réussite de l'expérience. Le pompage optique s'est cependant révélé moins efficace que prévu. Nous avons par suite été contraints d"opérer avec des tensions de vapeur de rubidium élevées; la relaxation par collisions d'échange devient alors dominante, et fait perdre aux enduits paraffinés une partie de leur intérêt. Les performances moins bonnes du système atomique ont augmenté les exigences concernant la réalisation de la cavité, et les problèmes technologiques en sont devenus d'une complexité telle que nous n’avons pas encore pu les maîtriser entièrement. Nous avons observé l'amplification maser d'une onde à 3.035,7 MHz pour la première fois en Janvier 1966; devant les difficultés rencontrées pour atteindre les conditions d'oscillation, nous avons été amenés à faire une étude systématique de l'amplification, tant sur le plan théorique que sur le plan expérimental $\left({ }^{7}\right)$. Les résultats ainsi obtenus permettent d'optimiser successivement les diverses parties du montage; le meilleur réglage actuel nous situe à $88 \%$ du seuil d'oscillation, valeur que la technique de mesure employée fait apparaitre comme un minimum et qui est encore susceptible d’être améliorée. A ce stade, il a été facile de réaliser un oscillateur en insérant la cavité dans une boucle de réaction comprenant un amplificateur destiné à compenser partiellement les pertes $\left({ }^{8}\right)$ : l'appareil ainsi obtenu présente également une stabilité de fréquence remarquable,

Dans le premier chapitre de ce mémoire, nous définirons le système atomique envisagé et préciserons les observables qui nous intéresseront par la suite, parmi lesquelles la cohérence hyperfine joue un rôle essentiel. Les méthodes permettant d'étudier ces observables seront passées en revue dans le deuxième chapitre. L'amplification maser par la transition 0-0, I'approche à l'oscillation et la possibilité d'obtenir celleci avec un circuit de réaction extérieur feront l'objet des troisième et quatrième chapi-

$$
\ldots 1 \ldots
$$


tres. Les deux derniers chapitres exposeront enfin l'application de l'étude générale précédente au cas du rubidium 85 et les résultats expérimentaux correspondants. Le développement de certains points sera détallie en appendice. 


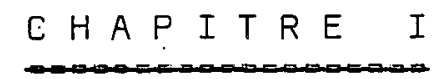

\section{LE SYSTEME ATOMIQUE \\ 000000000000000000000}

Nous nous proposons d'étudier le système constitué par la vapeur monoatomique d'un métal alcalin, enfermé dans, une cellule à une température voisine de l'ambiante. Les atomes, dans leur état fendamental, se déplacent d'un point à l'autre de la cellule. Au cours de ce mouvement, ils subissent des collisions entre eux, contre les parois de la cellule et éventuellement contre les molécules d'un gaz étranger, collisions qui modifient profondément leurs trajectaires et peuvent, d'autre part, être à l'origine de divers phénomènes de relaxation. Nous les supposerons de plus soumis à différentes perturbations extérieures :

- Présence d'un champ magnétique statique.

- Action d'une onde lumineuse, produisant un pompage optique.

- Application d'un champ magnétique de radiofréquence, susceptible de créer des cohérences hyperfines.

L'état fondamental des atomes alcalins est un état ${ }^{2} S_{1 / 2}(L=0, J=S=1 / 2)$. Le noyau possède un spin nucléaire I; la multiplicité du système est donc $2(2 I+1)$.

Le champ magnétique statique $\vec{H}_{0}$ sera supposé uniforme, et nous prendrons sa direction comme axe $\mathrm{Oz}$. Ce cas mis à part, les perturbations "vues" à un instant donné par deux atomes différents ne sont en général pas les mêmes : elles dépendent de ieur position, de leur vitesse et de leur histoire antérieure, La description du système atomique apparait ainsi comme très compliquée : nous la simplifierons considérablement en faisant les deux hypothèses fondamentales suivantes :

a) L'ensemble des. atomes peut être représenté par une matrice densité moyenne $\sigma$. 
b) Lorsqu'un champ de radiofréquence $\vec{H}$ est appliqué à.la cellule, les atomes "voient" celui-ci, quel que soit leur mouvement, comme un "champ moyen" uniforme, linéaire et parallèle à $0 z$, de la forme $H_{1 z} \cos \omega t$.

La première hypothèse est inspirée par les résultats de l'étude du pompage optique et de la relaxation dans les systèmes tels que le nôtre ( ${ }^{9}$ ); nous reviendrons, au chapitre VI, sur sa validité. La seconde peut sembler plus surprenante. Nous nous intéressons en effet à des champs $\vec{H}$ dont la fréquence est de quelques gigahertz. Dans le cas d’un atome libre, l'élargissement Doppler dû à l'agitation thermique atteint alors plusieurs kilohertz, et semblerait devoir constituer la principale contribution à la largeur des résonances hyperfines; or l'hypothèse ci-dessus revient à le négliger, puisque l'expression du champ "vu" par les atomes ne contient aucun terme de déphasage lié au mouvement. Nous justifierons plus loin (Appendice II) sa validité dans le cas de nos expériences, et calculerons alors la relation liant $H_{1 z}$ à $\vec{H}$; pour l'instant, il nous suffit de considérer $H_{1 z}$ comme un paramètre proportionnel à l'amplitude du champ appliqué $\vec{H}$.

Dans ces conditions, la matrice densité o évolue suivant l'équation :

$$
\frac{d \sigma}{d t}=-i\left[\mathscr{H}_{0}+\mathscr{H}_{1}, \sigma\right]+\mathscr{P}+\mathcal{R}
$$

○பे

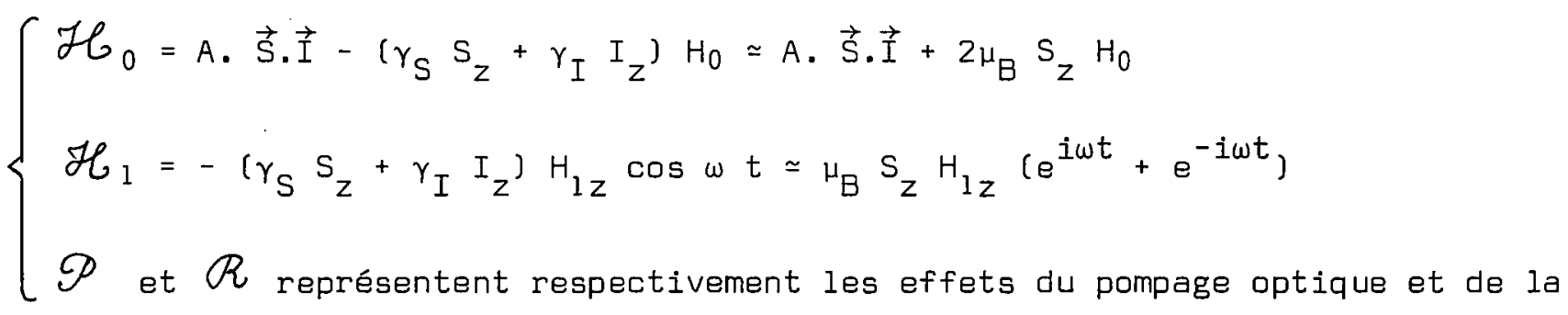
relaxation.

Sous l'influence du couplage hyperfin $A . \vec{S} . \vec{I}, 1$ létat fondamental se sépare en deux niveaux de nombres quantiques $F_{ \pm}=I \pm 1 / 2$, distants de $\nu_{a}=\omega_{a} / 2 \pi=\left(E_{F_{+}}-E_{F_{-}}\right) / h$ dans l'échelle des fréquences. L'action de $\vec{H}_{0}$ lève la dégénérescence de ces deux niveaux 


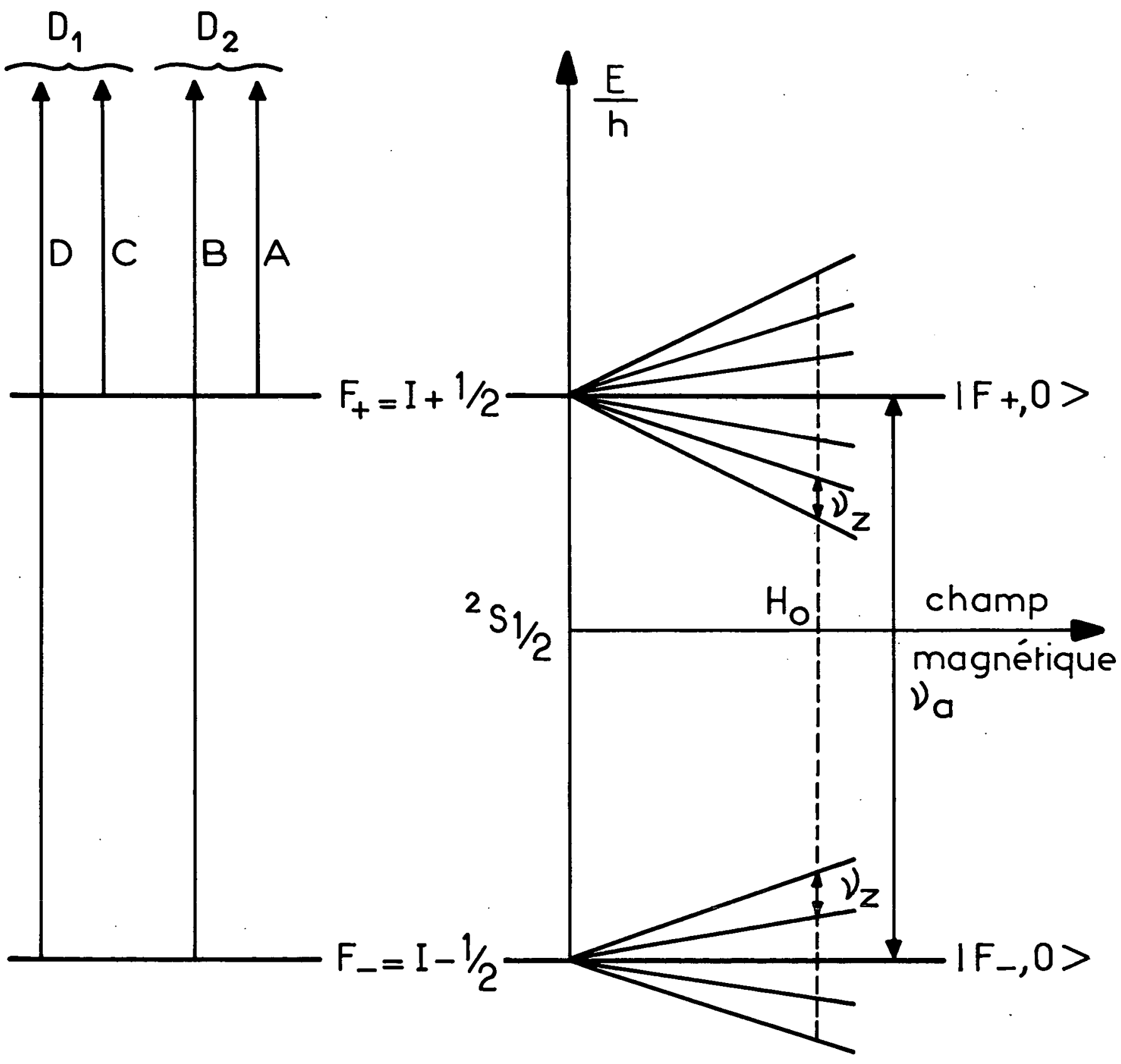

Fig. 1 
et les sépare en sous-niveaux Zeeman $\mid F, \mu_{F}>$ distants de $v_{Z}=g_{F} \mu_{B} H_{0} /$ h. Nous nous intéressons aux isotopes possédant un spin nucléaire I demi-entier; il existe alors deux sous-niveaux $\mid F_{+}, 0>$ et $\mid F_{-}, 0>$ dont l'écart, ou "fréquence 0-0" reste au premier ordre indépendant du champ et égal à $\nu_{a}$ (fig. 1). Les processus de relaxation (au sens large) agissant sur les atomes confèrent aux sous-niveaux une certaine largeur; nous supposerons que $H_{0}$, quoique faible, a une valeur suffisante pour que ceux-ci soient résolus, ce qui ne nécessite en pratique que des chemps très inférieurs au champ terrestre. Toutes les observables relatives au système śtudié peuvent être calculées une fois connus les éléments de matrice $\sigma_{\mu \mu}$.. L'interprétation des expériences effectuées en l'absence de radiofréquence ne nécessitera que la connaissance des éléments diagonaux ou populations $\sigma_{\mu \mu}$. Nous supposerons réalisée, dans toute la suite, la condition $\omega \simeq \omega_{a}$; l'hypothèse faite ci-dessus sur la séparation des sous-niveaux entraîne alors que les seuls éléments non diagonaux (cohérences) à considérer en présence de radiofréquence sont $\left\langle F_{+}, 0|\sigma| F_{-}, 0>=\sigma_{+}\right.$et son complexe conjugué $\sigma_{-+}$. Nous paserons $\left\langle F_{+}, 0|\sigma| F_{+}, 0\right\rangle=\sigma_{++}$et $\left\langle F_{-}, 0|\sigma| F_{-}, 0\right\rangle=\sigma_{--}$. Un calcul simple à partir de l'équation d'évolution (1) nous donne, sachant que $\left(S_{z_{+-}}=\left(S_{z_{-+}}\right)_{--1 / 2}(10)\right.$ :

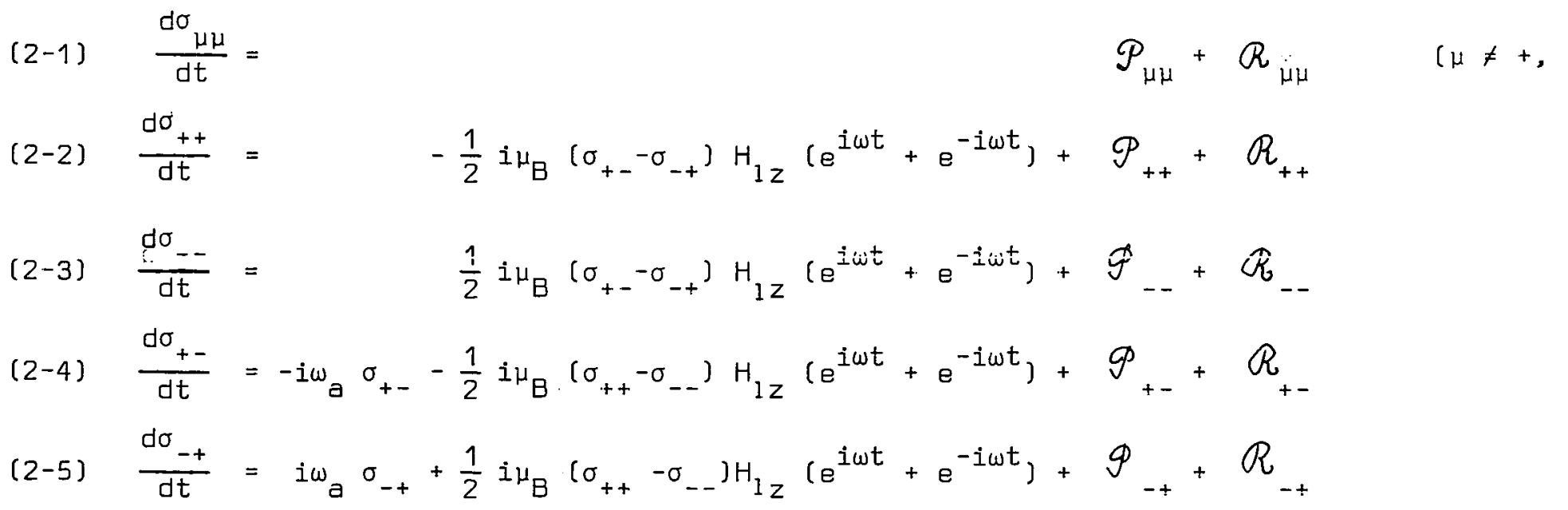


Il nous faut maintenant préciser un peu plus la forme de $\mathscr{P}$ et $\mathcal{R}$. Nous allons supposer que le pompage optique n'agit que sur les populations et n'introduit donc aucune cohérence dans le système : c'est le cas du "pompage hyperfin" que nous étudierons en détail plus loin. La relaxation liée aux collisions contre des parois recouvertes de paraffine et la relaxation liée aux collisions contre les molécules d'un gaz étranger sont des interactions aléatoires faibles moyennées par le mouvement ( ${ }^{9}$ ), qui ne couplent pas les éléments diagonaux aux éléments nọ diagonaux de $\sigma$. Sous l'action des seules causes précédentes et en l'absence de radiofréquence, les éléments non diagonaux $\sigma_{+-}$et $\sigma_{-+}$ s'amortiraient donc exponentiellement avec une seule constante de temps.

L'action des collisions d'échange entre les atomes de l'alcalin, phénomène très important comme nous le verrons, est par contre plus complexe. Une étude détaillée (11) (12) montre que, dans notre cas, :

- Les populations < $F_{+}, \pm 1|\sigma| F_{+}, \pm 1>,<F_{-}, \pm 1|\sigma| F_{-}, \pm 1>$, $\sigma_{++}$et $\sigma_{--}$sont perturbées par un terme proportionnel à $\left|\sigma_{+-}\right|^{2}$;

- La cohérence $\sigma_{+-}$évolue, sous l'effet des collisions d'échange; suivant l'équation :

$$
\begin{aligned}
{\left[\frac{d \sigma_{+-}}{d t}\right)_{\text {éch }}=-\frac{\sigma_{+-}}{T_{e}} } & {\left[\frac{3}{4}-\frac{I+3 / 2}{8(I+1 / 2)}\left(<F_{+}, 1|\sigma| F_{+}, 1>+<F_{+},-1|\sigma| F_{+},-1>\right)\right.} \\
& \left.-\frac{I-1 / 2}{8(I+1 / 2)}\left(<F_{-}, 1|\sigma| F_{-}, 1>+<F_{-},-1|\sigma| F_{-},-1>\right)-\frac{1}{4}\left(\sigma_{++}+\sigma_{--}\right)\right]
\end{aligned}
$$

Ce processus couple donc les éléments diagonaux avec l'élément non diagonal. Suivant une méthode classique $\left({ }^{13}\right)$, nous allons résoudre l'ensemble des équations d'évolution (2) par approximations successives, en les développant suivant les puissances croissantes de $\mathrm{H}_{1 z}$ supposé petit. Cette méthode va nous permettre d'aller assez loin dans l'étude du système atomique avec les seules hypothèses très larges que nous avons faites jusqu'ici; par contre, il est évident que nous ne pourrons pas traiter ainsi la 
saturation en présence d'un champ de radiofréquence d'intensité élevée. Nous obtenons les résultats ci-dessous.

Ordre 0. Nous posons $H_{1 z}=0$, et donc $\sigma_{+-}^{(0)}=\sigma_{-+}^{(0)}=0$. Les équations $(2-1)$, (2-2) et (2-3) se réduisent à :

$$
\left\{\begin{array}{l}
\frac{d \sigma_{\mu \mu}^{(0)}}{d t}=\mathscr{P}_{\mu \mu}^{(0)}+R_{\mu \mu}^{(0)} \\
\frac{d \sigma_{++}^{(0)}}{d t}=\mathscr{P}_{++}^{(0)}+R_{++}^{(0)} \\
\frac{d \sigma_{--}^{(0)}}{d t}=\mathscr{P}_{--}+\mathbb{R}_{--}^{(0)}
\end{array}\right.
$$

Leur résolution nous permet de calculer l'évolution des populations sous l'effet du pompage optique et de la relaxation : nous en verrons un exemple dans le chapitre suivant. Nous supposerons ici líétat stationnaire atteint, et appellerons $\hat{\sigma}_{\mu \mu}^{(0)}$, $\hat{\sigma}_{++}^{(0)}$ et $\hat{\sigma}_{--}^{(0)}$ les valeurs des populations à l'équilibre.

Ordre 1. Nous remplaçons, dans (2-4), (2-5) et (3), les populations par leur valeur d'équilibre à l'ordre 0. L'équation (3) devient à coefficients constants; l'évolution des éféments $\sigma_{+-}$et $\sigma_{-+}$sous l'action simultanée du pompage optique et de toutes les causes de relaxation est alors exponentielle, et l'on peut écrire :

$$
\begin{aligned}
& \mathscr{P}_{+-}^{(1)}+\mathscr{R}_{+-}^{(1)}=\frac{-\sigma_{+-}^{(1)}}{\mathrm{T}_{2}} \\
& \mathscr{P}_{-+}^{(1)}+\mathbb{R}_{++}^{(1)}=\frac{-\sigma_{-+}^{(1)}}{T_{2}}
\end{aligned}
$$


L'équation (2-4) prend la forme :

(5)

$$
\frac{d \sigma_{+-}^{(l)}}{d t}=-\left(\frac{1}{T_{2}}+i \omega_{a}\right) \sigma_{+-}^{(1)}-\frac{1}{2} i \mu_{B}\left(\hat{\sigma}_{++}^{(0)}-\hat{\sigma}_{--}^{(0)}\right) H_{1 z}\left(e^{i \omega t}+e^{-i \omega t}\right)
$$

dont la résolution nous donne :

$$
\sigma_{+-}^{(1)}=-\frac{1}{2} i \mu_{B}\left(\hat{\sigma}_{++}^{(0)}-\hat{\sigma}_{--}^{(0)}\right) e^{-\left(\frac{1}{T_{2}}+i \omega_{a}\right) t} \int_{t_{i}}^{t} H_{1 z}\left[e^{\left[\frac{1}{T_{2}}+i\left(\omega+\omega_{a}\right)\right] t^{\prime}}+e^{\left[\frac{1}{T_{2}}-i\left(\omega-\omega_{a}\right)\right] t}\right.
$$

L'hypothèse $\omega \simeq \omega_{a}$ entraîne que le terme en $\omega+\omega_{a}$ est négligeable par rapport au terme en $\omega-\omega_{a}$. Nous avons donc, en régime permanent :

$$
\hat{\sigma}_{+-}^{(1)}=-\frac{\frac{1}{2} i \mu_{B}\left(\hat{\sigma}_{++}^{(0)}-\hat{\sigma}_{--}^{(0)}\right) H_{1 z} e^{-i \omega t}}{\frac{1}{T_{2}}-i\left(\omega-\omega_{a}\right)}
$$

et de même :

$$
\hat{\sigma}_{-+}^{(1)}=\frac{\frac{1}{2} i \mu_{B}\left(\hat{\sigma}_{++}^{(0)}-\hat{\sigma}_{--}^{(0)}\right) H_{1 z} e^{i \omega t}}{\frac{1}{T_{2}}+i\left(\omega-\omega_{a}\right)}
$$

Ordre 2. Nous remplaçons de même, dans (2-1), (2-2) et (2-3), les éléments non diagonaux par leur valeur stationnaire à l'ordre 1. Nous obtenons :

$$
\begin{aligned}
& \text { (7-1) } \frac{d \sigma_{\mu \mu}^{(2)}}{d t}=\quad \mathscr{P}_{\mu \mu}^{(2)}+R_{\mu \mu}^{(2)} \\
& \text { (7-2) } \frac{d \sigma_{+ \pm}^{(2)}}{d t}=-\frac{1}{4} \mu_{\mathrm{B}}^{2}\left(\hat{\sigma}_{++}^{(0)}-\hat{\sigma}_{--}^{(0)}\right) H_{1 z}^{2} \frac{2 / T_{2}}{\left[\frac{1}{T_{2}}\right)^{2}+\left(\omega-\omega_{a}\right)^{2}}+\mathscr{P}_{++}^{(2)}+\mathbb{R}_{++}^{(2)} \\
& -\frac{1}{4} \mu_{B}^{2}\left(\hat{\sigma}_{++}^{(0)}-\hat{\sigma}_{--}^{(0)}\right) H_{1 z}^{2}\left[\frac{e^{2 i \omega t}}{\frac{1}{T_{2}}+i\left(\omega-\omega_{a}\right)}+\frac{e^{-2 i \omega t}}{\frac{1}{T_{2}}-i\left(\omega-\omega_{a}\right)}\right]
\end{aligned}
$$




$$
\begin{aligned}
& (7-3) \frac{d \sigma_{--}^{(2)}}{d t}=\frac{1}{4} \mu_{B}^{2}\left(\hat{\sigma}_{++}^{(0)}-\hat{\sigma}_{--}^{(0)}\right) H_{1 z}^{2} \frac{2 / T_{2}}{\left(\frac{1}{T_{2}}\right)^{2}+\left(\omega-\omega_{a}\right)^{2}}+\mathscr{P}_{-}^{(2)}+\mathcal{R}_{--}^{(2)} \\
& +\frac{1}{4} \mu_{B}^{2}\left(\hat{\sigma}_{++}^{(0)} \hat{\sigma}_{-}^{(0)}\right) H_{1 z}^{2}\left[\frac{e^{2 i \omega t}}{\frac{1}{T_{2}}+i\left(\omega-\omega_{a}\right)}+\frac{e^{-2 i \omega t}}{\frac{1}{T_{2}}-i\left(\omega-\omega_{a}\right)}\right]
\end{aligned}
$$

Nous n'avons besoin de connaître pour la suite que la valeur, en régime permanent, des populations modifiées par l'action de la radiofréquence. Nous avons vu plus haut que le terme perturbateur lié aux collisions d'échange, intervenant dans les éléments diagonaux de $R_{0}$, est proportionnel à $\left|\sigma_{+-}\right|^{2}$, soit ici à $\mu_{B}^{2} H_{1 z}^{2} /\left[\left(1 / T_{2}\right)^{2}+\left(\omega-\omega_{a}\right)^{2}\right]$. Le système (7) nous montre alors sans calculs que la variation des populations de tous les niveaux sous l'influence de la radiofréquence est proportionnelle à cette même quantité :

$$
\Delta \sigma_{\mu \mu}^{(2)} \propto \frac{\mu_{B}^{2}\left(\hat{\sigma}_{++}^{(0)}-\hat{\sigma}_{--}^{(0)}\right) H_{1 z}^{2}}{\left(\frac{1}{T_{2}}\right)^{2}+\left(\omega-\omega_{a}\right)^{2}}
$$

Ordres supérieurs.

Le calcul pourrait être continué de la même façon, apportant alternativement une correction aux éléments non diagonaux et aux éléments diagonaux de $\sigma$, mais il devient rapidement inextricable, surtout à cause des termes d'échange. Comme nous l'avions laissé prévoir, nous devons donc renoncer à traiter correctement la saturation. Nous pouvons toutefois en tenir compte de façon phénoménologique; par exemple, nous prévoyons qu'une itération répétée nous conduirait à un développement en série suivant les puissances de $H_{1 z}$ dont la somme ferait apparaître un terme d'élargissement en $\mathrm{H}_{1 \mathrm{z}}^{2}$ au dénominateur, suivant une expression de la forme : 


$$
\Delta \sigma_{\mu \mu}^{(\infty)} \propto \frac{\mu_{B}^{2}\left(\sigma_{++}^{(0)}-\sigma_{-}^{(0)}\right) H_{1 z}^{2}}{\left(\frac{1}{T_{2}}\right)^{2}+\left(\omega-\omega_{a}\right)^{2}+C H_{1 Z}^{2}}
$$

C étant une constante. Nous emploierons un tel procédé au chapitre IV.

$$
\text { Les expressions des différents éléments de matrice ainsi obtenues seront }
$$
utilisées dans les chapitres suivants. 
Nous nous proposons, dans ce chapitre, de décrire les méthodes permettant l'étude des diverses observables liées au système atomique envisagé, et plus particulièrement de celles qui font intervenir l'élément non diagonal $\sigma_{+-}$.

Cette étude peut être menée dans le cadre du montage expérimental de principe schématisé sur la figure 2 lnous reviendrons plus tard sur la constitution détaillée du montage réel). Le centre du dispositif est constitué par la cellule à vapeur alcaline soumise au champ magnétique statique $\vec{H}_{0}$. La raie de résonance de l'alcalin produite par la lampe traverse un filtre qui permet de donner à la lumière de pompage les caractéristiques désirées; on peut également insérer à cet endroit des écrans atténuant le flux lumineux dans un rapport connu. Un obturateur placé derrière le filtre nous donne la possibilité de couper la lumière ou de la rétablir en un temps très court. Le faisceau atteint ensuite la cellule, et le flux lumineux transmis est recueilli par un photodétecteur suivi d'un oscilloscope, dont la déviation verticale est ainsi proportionnelle à chaque instant à la quantité de lumière ayant traversé la cellule.

Celle-ci est contenue dans une cavité hyperfréquence accordée à une fréquence $\omega_{c}$ voisine de la fréquence atomique $\omega_{a}$. Un générateur hyperfréquence fournit une puissance $P_{i}$ à la fréquence $\omega$; une partie de cette puissance pénètre dans la cavité par un premier dispositif de couplage, eréant ainsi un champ oscillant $\vec{H}$ au niveau de la cellule. Le circulatbur permet de séparer et d’étudier la puissance réfléchie $P_{r}$; on peut aussi mesurer la puissance $P_{s}$ transmise par la cavité à travers un second dispositif de couplage. 
Nous passerans en revue quatre types d'expériences que. l'on peut effectuer : étude de l'absorption lumineuse en l'absence de radiofréquence, détection optique de la résonance 0-0, modulations lumineuses hyperfines, détection radioélectrique de la résanance $0-0$.

\section{A) ETUDE DE L'ABSORPTION LUMINEUSE EN L'ABSENCE DE RADIOFREQUENCE}

Les méthodes n'utilisant pas de radiofréquence ne nous donnent pas d'informations directes sur la cohérence hyperfine, puisque celle-ci n'est pas excitée. Nous allons cependant voir que l'étude de la variation de la lumière absorbée au cours du temps, à la suite d'une modification brusque de l'intensité d'illumination, nous apporte sur le système atomique des renseignements particulièrement intéressants.

Le problème du pompage optique et de la relaxation dans un tel système est assez compliqué, en particulier lorsque l'intensité lumineuse n'est pas uniforme dans toute la cellule $\left({ }^{14}\right)$. Il a fait l'objet d'une étude détaillée dans l'hypothèse où l'absorption optique par la vapeur alcaline est faible et l'intensité lumineuse uniforme $\left({ }^{9}\right)$. Nous allons en utiliser les résultats et pousserons les calculs dans un cas simple en nous attachant à mettre en évidence les variations de populations, quantités qui nous intéressent particulièrement ici.

Nous simplifierons le problème difficile de la répartition de la lumière dans la cellule en supposant que cette dernière est un cylindre d'axe Oz, illuminé par un faisceau parallèle à $0 z$ couvrant totalement et uniformément la face d'entrée (fig. 2) . Dans le cas général, l'intensité et la composition spectrale de la lumière de pompage varient le long de $\mathrm{Oz}$ par suite de l'absorption, et il est très difficile dans ces conditions de relier les observables qui nous intéressent aux signaux mesurés. Nous supposerons donc que l'absorption optique par la vapeur alcaline est faible; les conditions d'illumination sont alors uniformes en tous les points de la cellule. 


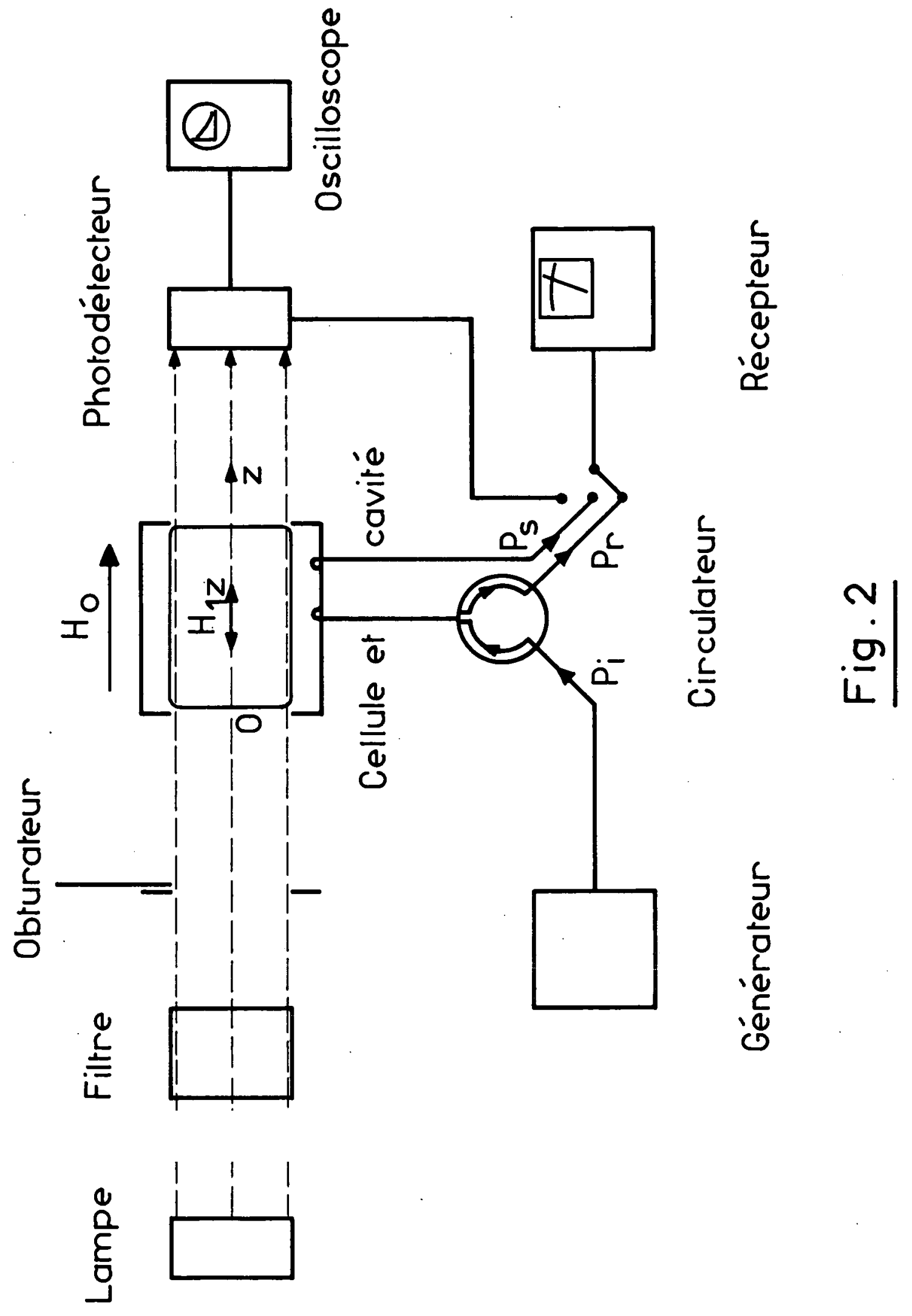


Il nous faut maintenant préciser la composition de la lumière utilisée. Nous effectuerons le calcul dans l'hypothèse du "pompage hyperfin". Rappelons que la raie de résonance optique comporte les deux composantes de structure fine $\mathrm{D}_{1}\left({ }^{2} \mathrm{~S}_{1 / 2} \rightarrow{ }^{2} \mathrm{P}_{1} / 2\right)$ et $\mathrm{D}_{2}\left({ }^{2} \mathrm{~S}_{1 / 2} \rightarrow{ }^{2} \mathrm{P}_{3 / 2}\right)$. Les structures hyperfines de $1^{\prime}$ état ${ }^{2} \mathrm{P}$ sont en général inférieures à la largeur Doppler, tandis que celle de l'état ${ }^{2} S_{1 / 2}$ lui est en général supérieure. Chacune des raies $D_{1}$ et $D_{2}$ se sépare dono en deux composantes hyperfines dues à la structure de l'état fondamental. La raie de résonance comprend ainsi au total quatre composantes dont l'intensité (en photons par seconde) au niveau de la cellule sera notée par A, B, C, D [fig. 1). Le pompage hyperfin que nous allons envisager consiste à illuminer la vapeur par les raies $D_{1}$ et $D_{2}$ non polarisées dans lesquelles on a rendu $B \gg P$ et $D \gg C$; nous verrons plus loin comment, dans un cas particulier, un filtrage de la lumière émise par la lampe permet d'arriver à ce résultat. Tous les atomes situés dans le niveau $F_{+}$ont alors une même probabilité d'excitation $1 / T_{p_{+}}$et ceux situés dans le niveau $F_{-}$ont une probabilité d'excitation très supérieure, $1 / T_{P_{-}}$. Les atomes portés dans l'état excité $2 \mathrm{P}$ y restent pendant un temps moyen égal à la durée de vie de cet état (quelques $10-8$ secondes puis retombent dans l'état fondamental en se partageant entre les deux niveaux $F_{+}$et $F_{-}$; le résultat global du cycle est donc un transfert de populations vers le niveau pour lequel l'absorption optique est la plus faible, c'est-à-dire de $F_{-}$vers $F_{+}$. Avec le pompage optique ainsi défini, la lumière absorbée par la vapeur $L_{A}$ est une fonction linéaire de l'observable $<\vec{S} . \vec{I}>\left({ }^{15}\right)$. Dans le cas d'une cellule ne contenant pas de gaz étranger, cetite ubservátie relaxe avec unc seule constante de temps; par contre, en présence d’un gaz étranger, $<\vec{S} . \vec{I}>$ relaxe avec une infinité dénombrable de constantes de temps associées chacune à un mode de diffusion dans le gaz vers la paroi $\left({ }^{9}\right)$. Une approximation qui nous suffira consiste à ne tenir compte que du premier d'entre eux, largement prépondérant. Nous considérerons donc que $\vec{S}$. $\vec{I}>$ relaxe dans tous les cas avec une seule constante de temps T.1H' que l'on peut écrire ...... 
sous la forme :

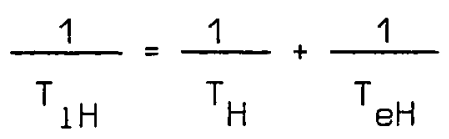

où $T_{H}$ représente l'effet de la parai et du gaz s'il existe, tandis que $T_{e H}$ représente la contribution due aux collisions d'échange entre les atomes de l'alcalin.

L'évolution de $\langle\vec{S} \cdot \vec{I}\rangle$ sous l'action simultanée du pompage optique et de la relaxation reste cependant compliquée et fait en général intervenir plusieurs constantes de temps. Cette évolution étant différente en l'absence ou en présence d'un gaz étranger dans la cellule, nous allons considérer ces deux cas successivement.

a) Cellule sans gaz étranger.: : Pendant son séjour dans l'état excité, l'atome ne subit alors aucune collision; porté dans un sous-niveau donné de l'état excité à la suite du processus d'absorption, il y reste jusqu'à la retombée vers l'état fondamental par émission spontanée. Le résultat global du pompage optique dans ces conditions ["pompage Kastler"] peut être calculé connaissant le tableau des éléments de matrice ou des probabilités de transition optiques. On trouve, comme il est normal, que le niveau $F_{+}$s'enrichit aux dépens du niveau $F_{-}$, mäis que la présence des composantes hyperfines de la raie $D_{2}$ produit en outre un alignement, c'est-à-dire une répartition de populations entre le sous-niveau $\mid F_{+}, \mu_{F_{+}}>$telle que le sous-niveau $\mid F_{+}$, $0>$ soit le moins peuplé et les sous-niveaux $\mid F_{+}, \mu_{F_{+}}= \pm F_{+}>$les plus peuplés.

b) Cellule avec gaz étranger : Si la cellule contient un gaz étranger sous une pression de quelques torrs, un-atome alcalin subit en moyenne plusieurs collisions contre les molécules de gaz étranger pendant la durée de son séjour dans l'état excité. Ces collisions peuvent être à l'origine de divers transferts de populations : quenching (retombée non radiative vers l'état fondamental), passage des atomes de l'état 
${ }^{2} \mathrm{P}_{1 / 2}$ vers 1 'état ${ }^{2} \mathrm{P}_{3 / 2}$ et réciproquement, etc. Si la pression de gaz étranger est suffisante, il se produit une égalisation des populations de tous les sous-niveaux Zeeman de l'état excité ${ }^{2} P$ ("pompage Dehmelt") ( $\left.{ }^{16}\right)$; les atomes ont alors une probabilité égale de retomber, radiativement ou non, dans chaque sous-niveau $\mid F, \mu_{F}>$ de l'état fondamental. Le résultat global est ici encore un enrichissement du niveau $F_{+}$aux dépens du niveau

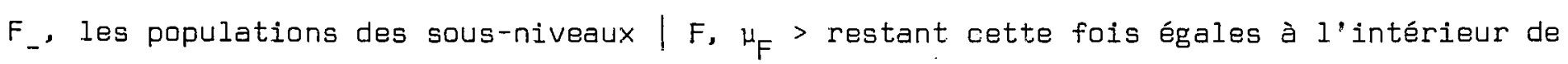
chaque niveau $F$.

Que la cellule contienne ou non un gaz étranger, il est ainsi possible d'établir une inversion de populations entre les sous-niveaux $\mid F_{f}$, $0>$ et $\mid F_{-}, 0>$ et nous avons pu l'observer expérimentalement dans les deux cas. Comme nous le verrons dans l'appendice II, l'obtention d'une forte amplification maser par la transition 0-0 nécessite cependant la présence d’un gaz étranger, et nous allons poursuivre le calcul dans l'hypothèse d'un "pompage Dehmelt" (qui est d'ailleurs un peu plus efficace que le "pompage Kastler", puisque dans ce dernier cas le sous-niveau | $F_{+}$, 0 > est moins peuplé que les autres sous-niveaux de $F_{+}$).

Appelons n le nombre d'atomes alcalins par unité de volume de la cellule, $N_{+}=n \sum_{\mu F_{+}} \sigma_{\mu \mu}^{(0)}$ la population du niveau $F_{+}$par unité de volume, $N_{-}=n \sum_{\mu_{-}} \sigma_{\mu \mu}^{(0)}$ celle du niveau F_. On a :

$$
\begin{aligned}
\langle\vec{S} \cdot \vec{I}\rangle & =\operatorname{Trace}(\sigma \vec{S} \cdot \vec{I})=\frac{I}{2} \sum_{\mu_{F}} \sigma_{\mu \mu}^{(0)}-\frac{I+1}{2} \sum_{\mu_{F}} \sigma_{\mu \mu}^{(0)} \\
& =\frac{1}{n}\left(\frac{I}{2} N_{+}-\frac{I+1}{2} N_{-}\right)
\end{aligned}
$$

La lumière absorbée $L_{A}$ (en photons par seconde) et sa variation sant données par : 


$$
\left\{\begin{array}{l}
L_{A}=\frac{N_{+}}{T_{P_{+}}}+\frac{N_{-}}{T_{P_{-}}} \\
\frac{d L_{A}}{d t}=\frac{1}{T_{P_{+}}} \frac{d N_{+}}{d t}+\frac{1}{T_{P_{-}}} \frac{d N_{-}}{d t}
\end{array}\right.
$$

En appelant $N$ la population totale de l'état excité, la conservation du nombre d'atomes"s'écrit :

$$
N_{+}+N_{-}+N=n
$$

Nous supposerons par ailleurs le facteur de Boltzmann égal à l, c'est-à-dire que le rapport $\tilde{N}_{+} / \tilde{N}_{-}$des populations des niveaux $F_{+}$et $F_{-}$à l'équilibre thermique est égal au rapport $(2 I+2) /(2 I)$ de leurs poids statistiques. En désignant par $\tau$ la durée de vie de l'état excité, l'évolution des populations sous l'action du pompage optique et de la relaxation obéit aux équations suivantes :

$$
\left\{\begin{array}{l}
\frac{d N_{+}}{d t}=-\frac{N_{+}}{T_{P_{+}}}-\left[\frac{N_{+}-\frac{2 I+2}{2(2 I+1)}(n-N)}{T_{1 H}}\right]+\frac{2 I+2}{2(2 I+1)} \frac{N}{\tau} \\
\frac{d N_{-}}{d t}=-\frac{N_{-}}{T_{P_{-}}}-\left[\frac{N_{-}-\frac{2 I}{2(2 I+1)}(n-N)}{T_{1 H}}\right]+\frac{2 I}{2(2 I+1)} \frac{N}{\tau} \\
\frac{d N}{d t}=\frac{N_{+}}{T_{P_{+}}}+\frac{N_{-}}{T_{P_{-}}}-\frac{N}{\tau}
\end{array}\right.
$$

La durée de vie $\tau\left(\sim 10-8\right.$ s) est courte par rapport à $T_{P_{+}}$et $T_{p_{-}}$ ( $>10^{-3} \mathrm{~s}$ ), ce qui entraîne $N \ll \pi$. La résolution des équations ( 9 ) nous donne, en affectant d'un $\wedge$ les valeurs d'équilibre et en négligeant $\tau$ et $N$ dans le résultat final: 
$(10-1)$

$$
\begin{aligned}
& \hat{N}_{+}=(2 I+2) \pi \frac{\frac{1}{T_{P_{-}}}+\frac{1}{T_{1 H}}}{\frac{2 I}{T_{P_{+}}}+\frac{2 I+2}{T_{P_{-}}}+\frac{2(2 I+1)}{T_{1 H}}} \\
& \hat{N}_{-}=2 I \quad \pi \frac{\frac{1}{T_{P_{+}}}+\frac{1}{T_{1 H}}}{\frac{2 I}{T_{P_{+}}}+\frac{2 I+2}{T_{P_{-}}}+\frac{2(2 I+1)}{T_{1 H}}}
\end{aligned}
$$

$$
\hat{L}_{A}=\frac{\hat{N}_{+}}{T_{P_{+}}}+\frac{\hat{N}_{-}}{T_{P_{-}}}=n \frac{(2 I+2) T_{P_{+}}+2 I T_{P_{-}}+2(2 I+1) T_{1 H}}{2(2 I+1) T_{P_{+}} T_{P_{-}}+2 I T_{1 H} T_{P_{-}}+(2 I+2) T_{1 H} T_{P_{+}}}
$$

$$
\frac{d L_{A}}{d t}=-\frac{1}{\tau_{p}}\left(L_{A}-\hat{L}_{A}\right) \quad \text { avec : }
$$

$$
\frac{1}{\tau}=\frac{2 I}{2(2 I+1) T_{P_{+}}}+\frac{2 I+2}{2(2 I+1) T_{P_{-}}}+\frac{1}{T_{1 H}}=\frac{1}{T_{P}}+\frac{1}{T_{1 H}}
$$

en introduisant un temps de pompage effectif $T_{p}$, inversement proportionnel à l'intensité d'illumination. La variatiof etesurs.du temps de la lumière absorbée est donc exponentielle; si le faisceau pompant est démasqué brusquement à l'instant 0 et tombe sur un système en équilibre thermique, la lumière absorbée $\tilde{L}_{A}$ au début du pompage est donnée par :

$(10-6)$

$$
\tilde{L}_{A}=\frac{n}{2(2 I+1)}\left(\frac{2 I+Z}{T_{P_{+}}}+\frac{2 I}{T_{P_{-}}}\right)
$$

Un cas particulier très intéressant de ces équations, dans lequel il est en général possible expérimentalement de se placer, est celui où le temps de pompage $T_{p}$ est beaucoup plus court que tous les temps de relaxation relatifs aux différentes observables du système atomique ("saturation lumineuse"). En appelant $n_{+}=n \sigma_{++}^{(0)} 1 a$ 
population du sous-niveau $\mid F_{+}, 0>$ et $n_{-}=n \sigma_{--}^{(0)}$ celle du sous-niveau $\mid F_{-}$, $0>$, on peut alors écrire :

$(11-1)$

$$
\begin{aligned}
\hat{N}_{+} & =(2 I+2) n_{+} \\
N_{-} & =2 I \frac{n_{-}}{-\frac{1}{T_{P_{+}}}+\frac{1}{T P_{-}}} \\
\hat{n}_{+}-\hat{n}_{-} & =n \frac{2 I}{T_{P_{+}}+\frac{2 I+2}{T_{P_{-}}}} \\
\hat{L}_{A} & =n \frac{2(2 I+1)}{2 I T P_{-}+(2 I+2) T_{P_{+}}}
\end{aligned}
$$

Les équations (10), ou leur cas particulier (11), déterminent entièrement l'évolution du système atomique. Comme nous le verrons dans un instant, (10-4) et (10-5) nous permettent, par l'étude de la lumière absorbée et de sa variation, de connaitre les quantités très intéressantes $T_{p}^{\prime}$ et $T_{1 H}$ Les populations, par contre, sont données par $(10-1),(10-2)$ et $(11-1)$ en fonction des paramètres $T_{P_{+}}$et $T_{P_{-}}$. Pour achever le calcul, deux méthodes sant possibles.

a) $T_{P_{+}}$et $T_{P_{-}}$peuvent en principe être déterminés. Désignons par $Q$ la section efficace d'absorption des photons pour l'ensemble de la raie de résonance optique; compte tenu des poids statistiques respectifs des différents niveaux, on peut écrire:

$$
\begin{aligned}
& \frac{1}{T P_{+}}=\frac{Q}{6(2 I+1)}(C+2 A) \\
& \frac{1}{T P_{-}}=\frac{Q}{6(2 I+1)}(D+2 B)
\end{aligned}
$$

A, B, C, D étant les intensités des quatre composantes hyperfines, comme il a été expliqué plus haut. La connaissance de $Q$ et la mesure de $A, B, C, 0$ nous permettent donc de connaître $T_{P_{+}}$et $T_{P_{-}}$; mais le procédé est manifestement compliqué et peu précis. 


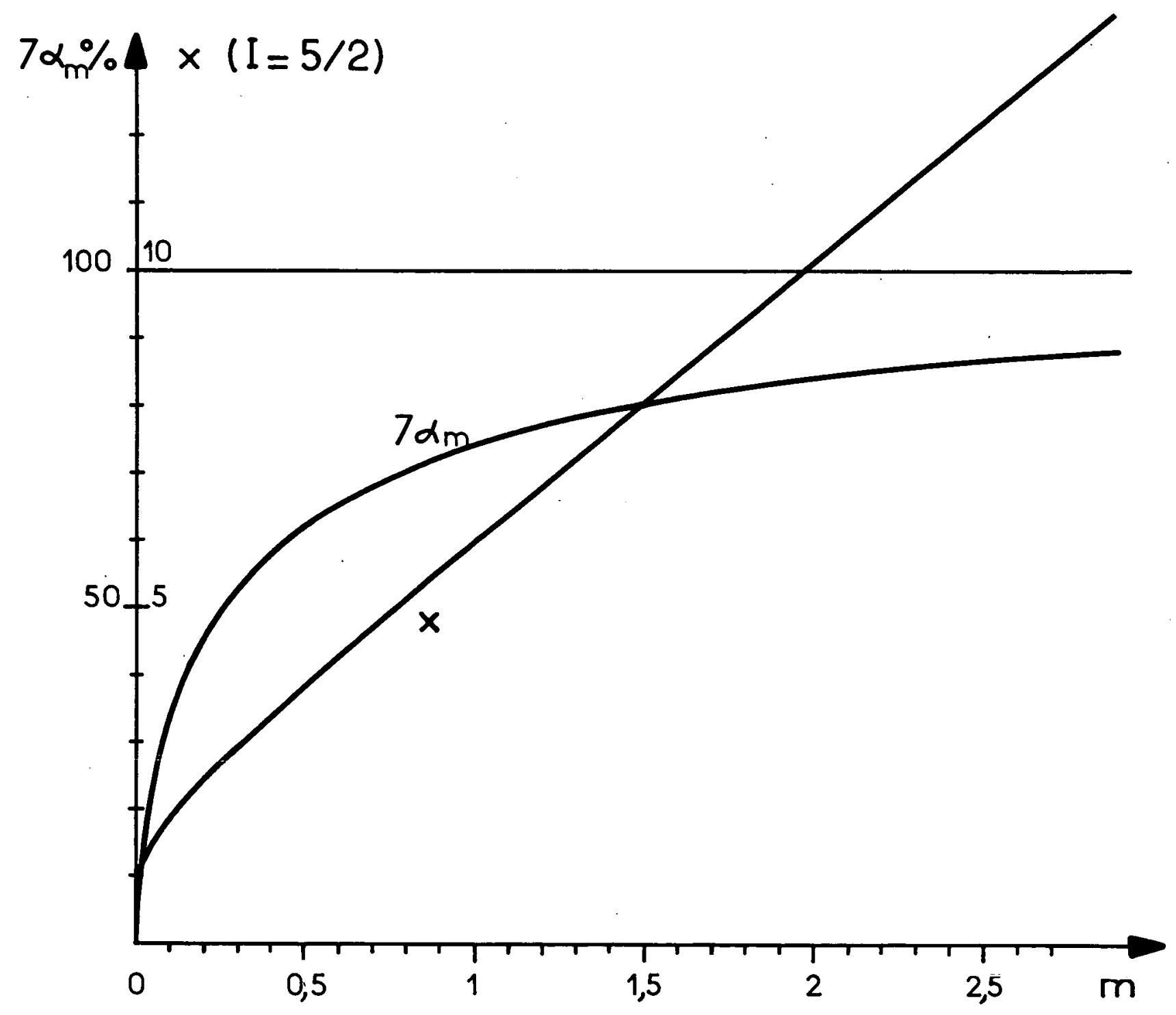

Fig. 3 
b) Une méthode bien préférable consiste à se placer dans le cas de la saturation lumineuse envisagé ci-dessus. $T_{P_{+}}$et $T_{P_{-}} n^{\circ}$ interviennent alors plus que par leur rapport $T_{P_{+}} / T_{P_{-}}=x$, qui peut être considéré comme un paramètre définissant la qualité de la lumière de pompage. Formons alors la quantité $m=\left(\hat{L}_{A}-\hat{L}_{A}\right) / \hat{L}_{A}$, quotient de l'amplitude de l'exponentielle de pompage (fig. 13) par la lumière absorbée en fin de pompage. Les deux quantités $x$ et $\alpha_{m}=\left(\hat{n}_{+}-\hat{n}_{-}\right) / n$ peuvent s'exprimer en fonction de $\mathrm{m}$; on obtient :

$$
x=1+\frac{2(2 I+1)}{(2 I+2) 2 I}\left[(2 I+1) m+\sqrt{(2 I+1)^{2} m^{2}+(2 I+2) 2 I m}\right]
$$

$$
\alpha_{m}=\frac{x-1}{(2 I+2) x+2 I}=\frac{-m+\sqrt{(2 I+1)^{2} m^{2}+(2 I+2) 2 I m}}{(2 I+2) 2 I(m+1)}
$$

Dans le cas où la "relaxation optique" est dominante, nous avons ainsi la possibilité très intéressante de déterminer la qualité de la lumière et l'inversion de populations entre les sous-niveaux bordant la transition 0-0 en fonction des caractéristiques de l'absorption lumineuse. La figure 3 représente la variation de $x$ et $(2 I+2) \alpha_{m}$ en fonction de $m$, dans le cas de ${ }^{85} \mathrm{RD}(I=5 / 2)$; avec le pompage optique employé, ne créant aucune différence de populations entre les sous-niveaux Zeeman d'un même niveau $F$, on ne peut évidemment dépasser la valeur $\alpha_{m}=1 /(2 I+2)$ obtenue lorsque le niveau $F_{\text {_ }}$ est complètement vidé au profit du niveau $F_{4}$. L'étude du système atomique par cette méthode s'apèrera de la manière suivante.

a) Après avoir laissé le système atomique assez longtemps dans le noir, on établit brusquement le faisceau de pompage. La lumière absorbée évolue exponentiellement suivant 1 'équation $(10-4)$; on mesure la constante de temps $\tau_{p}$ (fig. 13). On 
répète l'expérience pour des valeurs croissantes du temps de pompage 'Tp' obtenues'en atténuant le flux lumineux incident dans des rapports connus. D'après (10-5), la variation de $1 / \tau_{p}$ avec le flux lumineux est représentée par une droite (fig. 14] dont la pente nous renseigne sur le flux lumineux effectif présent dans la cellule, proportionnel à $1 / T_{p}$, et dont l'ordonnée à l'origine est égale à $1 / T_{1 H}=1 / T_{H}+1 / T_{e H^{*}}$ Or on sait par ailleurs $\left({ }^{1}\right)(12)$ que le temps de relaxation d'échange $T_{\text {eH }}$ est relié à la densité n d'atomes dans la cellule par l'équation $1 / T_{e H}=\bar{v} \pi \sigma^{2}$ éch $n$, où $\bar{v}$ désigne la vitesse relative moyenne d'agitation thermique et $\pi \sigma_{\text {ech }}^{2}$ la section efficace d'échange. La mesure de $1 / T_{1 H}$ pour une température de la cellule (c'est-à-dire une valeur de nj suffisamment basse, ou mieux l'extrapolation des mesures à n décroissant (fig. 16) nous fournit la contribution $1 / T_{H}$ à la relaxation de $<\vec{S} . \vec{I}>$ due aux collisions sur les parois et contre le gaz étranger; on en déduit la valeur de $T_{e H}$ à chaque température, ce qui permet de connaître la grandeur très importante qu'est la densité moyenne $n$ des atomes alcalins dans la cellule. Nous verrons en effet plus loin que la tension de vapeur existant dans nos cellules est en général très inférieure à la tension de vapeur saturante, et dépend en particulier de l'histoire antérieure de la cellule considérée : il est donc précieux de pouvoir la déterminer expérimentalement.

b) On se place dans les conditions de saturation lumineuse (forte illumination); on mesure $\tilde{L}_{A}$ et $\hat{L}_{A}$, et on forme la quantité $\left(\tilde{L}_{A}-\hat{L}_{A}\right) / \hat{L}_{A}=m$. L'équation (12-1) nous renseigne alors sur l'efficacité du filtrage hyperfin, tandis que I'équation (12-2) nous fournit $\alpha_{m}$, c'est-à-dire la différence relative de populations maximum qu'il est possible de maintenir entre les sous-niveaux $\mid F_{+}$, $0>$ et $\mathrm{F}_{-}, 0>$ avec le pompage optique employé. 
En résumé, cette étude nous permet de connaître :

- Le flux lumineux effectif présent dans la cellule, proportionnel à $1 / T_{p}$

- Le temps de relaxation $T_{H}$ de l'observable<S.I >, dû aux collisions contre les parois et le gaz étranger.

- Le temps de relaxation d'échange TeH' d'où l'on déduit la densité des atomes alcalins $\Pi_{0}$

- La qualité $x$ de la lumière de pompage.

- Une limite supérieure $\alpha_{m}$ de la différence de populations entre les sous-niveaux bordant la transition 0-0, intervenant en particulier dans l'expression de l'élément non-diagonal $\sigma_{+-}$.

Nous en verrons des exemples d'application dans la partie expérimentale. B) DETECTION OPTIQUE DE LA RESONANCE D-O

Nous supposons maintenant l'intensité d'illumination constante et soumettons la cellule à un champ $\vec{H}$ de fréquence de la transparence de la vapeur lorsque, l'amplitude de $\vec{H}$ (et donc $H_{1 z}$ ) ayant une valeur donnée, w varie lentement au voisinage de la fréquence de résonance $\omega_{a}$.

Nous avons vu au paragraphe précédent que, avec le pompage aptique employé, la lumière absorbée était donnée par :

$$
L_{A}=\frac{n}{T_{P_{+}}} \sum_{\mu_{F_{+}}} \sigma_{\mu \mu}+\frac{n}{T_{P_{-}}} \sum_{\mu_{F_{-}}} \sigma_{\mu \mu}
$$

Par ailleurs, nous avons établi au chapitre I que la variation des populations là l'ordre 2) de tous les niveaux sous l'action de la radiofréquence était proportionnelle à la quantité $\mu_{B}^{2}\left(\hat{\sigma}_{++}^{(0)}-\hat{\sigma}_{--}^{(0)}\right) H_{l z}^{2} /\left[\left(1 / T_{2}\right)^{2}+\left(\omega-\omega_{a}\right)^{2}\right]$. Il en est par .../... 
conséquent de même pour la variation $\Delta \mathrm{L}_{A}$ de la lumière absorbée et on peut écrire :

$$
\Delta L_{A} \propto \frac{\mu_{B}^{2}\left(\hat{\sigma}_{++}^{(0)}-\hat{\sigma}_{--}^{(0)}\right) H_{l z}^{2}}{\left(\frac{1}{T_{2}}\right)^{2}+\left(\omega-\omega_{a}\right)^{2}}
$$

$\Delta \mathrm{L}_{A}$ a donc la forme d'une courbe d'absorption lorentzienne, centrée sur la fréquence $\omega_{a}$ et de demi-largeur à mi-hauteur $\delta \omega_{a}=1 / T_{2}$. Nous pouvons ainsi déterminer la fréquence de résonance et le temps de relaxation de l'élément non diagonal $\sigma_{+-}$, qu'il est intéressant de comparer aux temps $\tau_{p}$ et $T_{1 H}$ dont nous avons. parlé au paragraphe précédent.

Notons que le signal $\Delta \mathrm{L}_{A}$ n'est autre que celui qui sert de base à toutes les horloges atomiques à rubidium commerciales ( ${ }^{1}$ ). C) MODULATIONS LUMINEUSES AUX FREQUENCES HYPERF INES

Supposons que le photodétecteur employé soit capable de répondre à des variations lumineuses aussi rapides que $\omega_{a}$. Nous pouvons nous demander, par analogie avec les nombreuses expériences de "faisceau croisé", quelles sont les conditions à remplir pour que l'absorption lumineuse soit effectivement modulée à cette fréquence. Notre formalisme très simple ne nous permet pas de répondre à cette question. Un calcul très général ( ${ }^{17}$ ) montre que la lumière transmise sera modulée à la fréquence de la transition 0-0 lorsqu'on excite cette dernière si le faisceau de pompage se propageant suivant $\mathrm{Oz}$ est polarisé circulairement. Comme on pouvait le prévoir, l'absorption lumineuse est alors sensible à l'élément non diagonal $\sigma_{+-}$et peut en permettre l'étude. La vérification expérimentale n'a pas encore été faite : des expériences analogues ont cependant été effectuées sur ${ }^{39} K$, dans le cas un peu différent d'une transition 1-1 (18). 


\section{D) DETECTION RADIOELECTRIQUE DE LA RESONANCE D-D}

Nous établissons encore dans la cavité un champ $\vec{H}$ dont nous maintenons l'amplitude constante. Ce champ crée dans l'ensemble des atomes paramagnétiques un moment magnétique oscillant $\vec{m}$, qu'il est facile de calculer en fonction de $H_{1 z}$ à l'aide des formules démontrées au chapitre I. On a en effet :

$$
\vec{M}=\frac{n}{K} \text { Trace }(\sigma \times \text { opérateur } \vec{M})=\frac{\mu_{B}}{K} n\left(\sigma_{+-}-\sigma_{-+}\right)
$$

soit, à l'ordre 1:

(14-1) $M=\frac{1}{2} i \frac{\mu_{B}^{2}}{k} n\left(\hat{\sigma}_{++}^{(0)}-\hat{\sigma}_{--}^{(0)}\right) H_{1 z}\left[\frac{e^{i \omega t}}{\frac{1}{T_{2}}+i\left(\omega-\omega_{a}\right)}-\frac{e^{-i \omega t}}{\frac{1}{T_{2}}-i\left(\omega-\omega_{a}\right)}\right]$

$(14-2)=\frac{\mu_{B}^{2}}{h} n\left(\hat{\sigma}_{++}^{(0)}-\hat{\sigma}_{--}^{(0)}\right) H_{1 z}\left[\frac{\left(\omega-\omega_{a}\right) \cos \omega t}{\left(\frac{1}{T_{2}}\right)^{2}+\left(\omega-\omega_{a}\right)^{2}}-\frac{\frac{1}{T_{2}} \sin \omega t \cdot}{\left(\frac{1}{T_{2}}\right)^{2}+\left(\omega-\omega_{a}\right)^{2}}\right]$.

$M$ comprend un terme en phase avec le champ $H_{1 z}$ (dispersion) et un terme en quadrature (absorption). Les susceptibilités correspondantes s'écrivent :

$(14-3)$

$$
\left\{\begin{array}{l}
x^{\prime}(\omega)=\frac{\mu_{B}^{2}}{k} n\left(\hat{\sigma}_{++}^{(0)}-\hat{\sigma}_{--}^{(0)}\right) \frac{\omega-\omega_{a}}{\left(\frac{1}{T_{2}}\right)^{2}+\left(\omega-\omega_{a}\right)^{2}} \\
x^{\prime \prime}(\omega)=-\frac{\mu_{B}^{2}}{k} n\left(\hat{\sigma}_{++}^{(0)}-\hat{\sigma}_{--}^{(0)}\right) \frac{1 / T_{2}}{\left(\frac{1}{T_{2}}\right)^{2}+\left(\omega-\omega_{a}\right)^{2}}
\end{array}\right.
$$

La présence dans la cavité d’un moment magnétique oscillant d'amplitude variable avec w modifiera ses propriétés. En particulier, la puissance réfléchie $P_{r}$ et la puissance transmise $P_{s}$ varieront au passage par la résonance $\omega=\omega_{a}$. 
Ce qui précède n’est évidemment autre que le schéma d'une expérience de résonance électronique classique sur la transition 0-0. Nous ne la décrirons pas davantage. En effet, nous nous proposons, au chapitre suivant, de traiter un cas plus général : au lieu de supposer, comme nous l'avons fait jusqu'à présent et comme il est d'usage dans les expériences de résonance habituelles, que l'amplitude du champ $\vec{H}$ est une constante imposée de l'extérieur, nous allons tenir compte de la réaction du système atomique sur le champ appliqué, qui peut aboutir en particulier à une amplification de celui-ci. Les résultats relatifs au cas $H$ = constante apparaitront alors comme des cas particuliers.

Notons qu'une classe très riche d'expériences est fournie par les techniques d’impulsions $\left({ }^{9}\right)$ : des séquences judicieusement choisies $d^{\prime} i m p u l s i o n s$ lumineuses et d'impulsions de radiofréquence permettent de mesurer une grande variété de temps de relaxation. Ces procédés sont en principe équivalents aux méthodes de détection en passage lent, en vertu des propriétés de la transformation de Fourier. En pratique, l'étude de la relaxation par les méthodes transitoires est souvent plus directe et parfois plus commode. 
Nous nous proposons dans ce chapitre d'étudier en détail l'interaction du système atomique avec le champ de radiofréquence, désignédans le référentiel du laboratoire par $\vec{H}$, sans considérer l'amplitude de celui-ci comme un paramètre fixé de l'extérieur.

Le problème est donc de relier les propriétés du système atomique, que nous avons établies dans les chapitres précédents en fonction du paramètre $H_{1 z}$, aux propriétés radioélectriques de la cavité contenant le système. Les.ealculs correspondants sont classiques en basse fréquence, où le circuit résonnant est du type LC : l'échantilIon se trouvant dans la self a une susceptibilité $x(\omega)$ variable avec la fréquence, donnée par des formules semblables à $(14-3)$, ce qui entraîne pour le eircuit résonnant des modifications dont l'expression est.facile à obtenir $\left({ }^{20}\right)$. Une méthade pour traiter le cas qui nous intéresse serait alors d'essayer de se ramener aux calculs déjà faits en basse fréquence, en définissant des circuits équivalents. Nous préfèrerens une descrïption électromagnétique de l'ensemele, plus physique à notre avis, que nous allons exposer maintenant.

\section{A) DEFINITIONS ET FORMULES GENERALES}

Comme nous l'avons dit, le champ $\vec{H}$ est produit dans une cavité entourant le système atomique. Une cavité électromagnétique est un volume diélectrique fermé limité par des parois conductrices (infiniment en première approximation). La résolution des équations de Maxwell dans le volume intérieur avec les eonditions aux limites ainsi 
imposées montre que le champ éleetromagnétique n’y.prend un valeur notable que pour des valeurs discrètes de. la longueur.d!onde (-longueurs.d'onde de.résenance: $\lambda_{c}$ ) et pour certaines configurations spatiales des champs électrique et magnétique appelées modes. Lorsque la cavité a une forme gémétrique simple, on peut à partir de ses dimensions calculer les longueurs d'onde $\lambda_{c}$, et en déduire les fréquences eorrespondantes si l'on connait la constante diélectrique $\varepsilon$ du milieu intérieur (dont nous supposerons pour la suite la perméabilité magnétique $\mu$ égale à-l'unités; of peut égałement déterminer pour chaque mode la carte du ehamp électrique $\vec{E}$-et du champ magnétique $\vec{H}$ en écrivant leurs composantes dans un système d'axes adapté-à la symétrie..du problème.

Dans un made donné, la cavité renferme une énergie éleetromagnétique :

$$
W=\frac{1}{8 \pi} \frac{\left.<\varepsilon E^{2}+\mu H^{2}\right\rangle_{\text {CaV }}}{2} V_{\text {cav }}=\frac{\left.<H^{2}\right\rangle_{\text {CaV }}}{8 \pi} V_{\text {cav }}
$$

où le signe < > cav désigne la valeur moyenne prise. sur toute la cavité de volume Vav Cette énergie étant supposée créée d’une manière ou d'une autre à un instant initial, ne peut s'y maintenir indéfiniment. En effet, si nous avens pu calculer les fréquences de résonance et les modes en supposant pour les parois une eonductivité infinie et à l'intérieur un diélectrique parfait, il y a toujours en réalité des pertes qui produiront une décroissanee exponentielle de:l'énergie emmagasìnée et sa transformation en chaleur. On caractérise ces pertes peur une cavité et un mode donnés en définissant un facteur de surtension $Q$, tel que l'énergie stockée ait été dívisée pap e $=2,718$ au bout de $Q / 2 \pi$ périodes. Un calcul simple conduit alors à la formule fondamentale suivante, que nous aurons souvent l'occasion.d'appliquer :

$$
P=\frac{\omega<H^{2}>_{\text {cav }} V_{\text {cav }}}{8 \pi Q}
$$

où $P$ est la puissance dássipée par toutes les causes de pertes. 
La cavité doit posséder un ou plusieurs dispositifs de couplage, permettant d'y établir le champ $\vec{H}$ et d'étudier les propriétés de l'efsemble. Nous considérerons le cas d'une cavité possédant un couplage d'entrée et un couplage de sortie reliés chacun à une ligne de transmüssion d'impédance earaetéristique $Z_{0}$. Ces couplages conduisent à un échange d'émergie entre la cavité.et. l’extérieur. Créons: à un instant initial une énergie électromagnétique $W$ à l intérieur de la cavité : l'ensemble étant laissé à lui-même, une puissance $\mathscr{P}_{e} s^{3}$ échappe:par le-couplage.d. entrée, une puissance $\mathscr{P}_{s}$ s'échappe par le couplage de sortie, et une puissance $\mathscr{P}_{0}$ est dissipée par effet Joule ou pertes diélectriques à l’intérieur de la cavité. En appliquant la formule (15) à chacune de ces puissances, on est amené- à distinguer une surtensien à vide $Q_{0}$ (en l'absence de couplage), deux surtensions de couplage $Q_{e e}$ et $Q_{e s}$ ' et une surtension en charge $Q_{C}$ reliée aux précédentes par $1 / Q_{C}=1 / Q_{0}+1 / Q_{e e}+1 / Q_{\text {es }} \cdot$ On définit enfin les coefficients de couplage par $\beta_{e}=Q_{0} / Q_{e e}$ et $\beta_{S}=Q_{0} / Q_{e s}$. La cavité étant accordée à la fréquence $\omega_{C} / 2 \pi=\nu_{C}$, la surtension $Q_{C}$, définissant son amortissement total, lui confère une largeur de bande $2 \delta \omega_{c} / 2 \pi=\Delta_{c}=v_{c} / Q_{c}=v_{c}\left(1+\beta_{e}+\beta_{s}\right) / Q_{0}$. L'intérêt des coefficients $Q$ ainsi introduits est qu'ils suffisent, connaissent $\omega_{c}$, à définir entièrement les propriétés électromagnétiques de la cavité : c'est ainsi que l'impédance d'entrée $Z_{i}$ de la cavité est dennée par :

$(16-1)$

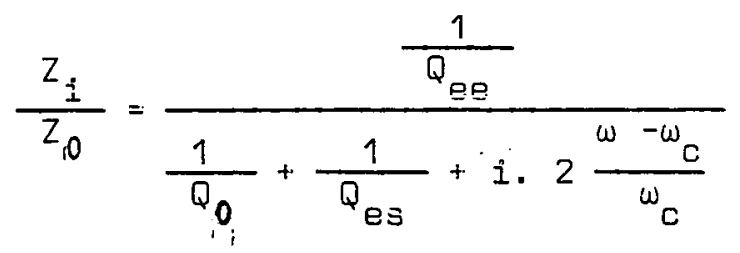

Il nous faut maintenant, et c'est le centre de toute cette question, déterminer les propriétés d'une cavité renfermant le système atomique, dont la présence se traduit par l'apparition dans la cavité du moment magnétique oscillant M calculé au chapitre précédent. Nous allons utiliser un résultat important établi par 
SLATER ( ${ }^{21}$ ) qui, à partir des équations de Maxwell avec terme source, a calculé les modifications apportées à une cavité par la présence dans celle-ci d'un moment électrique oscillant $\overrightarrow{\mathcal{P}}(\vec{r}, t)$ ou, par une transposition facile (22), d'un mament magnétique oscillant $\vec{m}(\vec{r}, t)=1 / 2 \vec{M}(\vec{r}) e^{i \omega t}+1 / 2 \vec{M}^{*}(\vec{r}) e^{-i \omega t}$, le champ magnétique étant, dans ce dernier cas, pris sous la forme $\vec{H}(\vec{r}, t)=1 / 2 \vec{H}(\vec{r}) e^{i \omega t}+1 / 2 \vec{H}^{*}(\vec{r}) e^{-i \omega t}$. Le calcul repose sur les deux hypothèses suivantes :

a) Le système physique associé à $\vec{m}$ est linéaire, cest-à-dire que sa "réponse" est

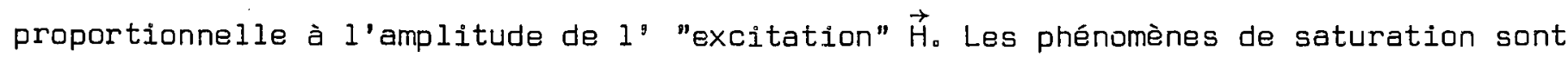
donc exclus.

b) La répartition spatiale du champ $\vec{H}$ (carte du mode de la cavité utilisé) n'est pas modifiée par l'interaction avec le moment oscillant.

Dans ces conditions, $I^{\circ}$ influence de $\vec{m}$ sur la cavité peut être représentée par un "facteur de surtension atomique" complexe $Q_{a}$; en particulier, la formule (16-1) donnant l'impédance d’entrée de la cavité est remplacée par $\left({ }^{2 l}\right)\left({ }^{23}\right)$ :

$(16-2)$

$$
\frac{Z_{i}}{Z_{0}}=\frac{\frac{1}{Q_{e e}}}{\frac{1}{Q_{0}}+\frac{1}{Q_{e s}}+\operatorname{Re} \frac{1}{Q_{a}}+i\left[2 \frac{\omega_{c} \omega_{c}}{\omega_{c}}+\operatorname{Im} \frac{1}{Q_{a}}\right]}
$$

Q étant donné par l'expression :

$$
\frac{1}{Q_{a}}=4 \pi i \frac{\int_{\text {cavité }} \vec{M}(\vec{r}) \cdot \vec{H}^{*}(\vec{r}) d V}{\int_{\text {cavité }} \vec{H}(\vec{r}) \cdot \vec{H}^{*}(\vec{r}) d V}
$$

Dans notre cas, nous avons établi au chapitre précédent la formule :

$$
M=\frac{1}{2} i \frac{\mu_{B}^{2}}{h} n\left(\hat{\sigma}_{++}^{(0)}-\hat{\sigma}_{--}^{(0)}\right)\left[\frac{H_{1 z}}{\frac{1}{T_{2}}+i\left(\omega-\omega_{a}\right)} e^{i \omega t}-\frac{H_{1 z}}{\frac{1}{T_{2}}-i\left(\omega-\omega_{a}\right)} e^{-i \omega t}\right]
$$

reliant la valeur du moment magnétique au paramètre $H_{1 z}$, dans l'hypothèse faite dès le $\ldots / 0$. 
début où $\sigma$ et $H_{1 z}$ sont des grandeurs moyennes relatives à l'ensemble des atomes. Nous écrirons donc ici :

$$
\frac{1}{Q_{a}}=-\frac{4 \pi \mu_{B}^{2}}{h} n\left(\hat{\sigma}_{++}^{(0)}-\hat{\sigma}_{--}^{(0)}\right) \frac{1}{\frac{1}{T_{2}} \div i\left[\omega-\omega_{a}\right]} n
$$

en définissant formellement un "coefficient de remplissage" n par la relation :

$$
\eta=\frac{\int_{\text {cavité }} \vec{H}_{1 z^{*}} \cdot \vec{H}(\vec{r}) d V}{\int_{\text {cavité }} H^{2}(\vec{r}) d V}
$$

L'expression exacte de $\eta$ et sa valeur numérique seront déterminées à l'appendice II, après avoir précisé la relation entre $H_{1 z}$ et $\vec{H}$ dans chacune des situations expérimentales auxquelles nous nous sommes intéressés (en particulier, présence ou absence $d^{\prime} u n$ gaz étranger). Nous pouvons cependant remarquer dès à présent que si

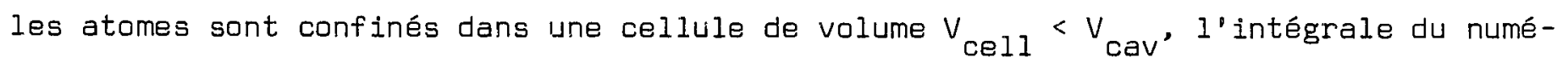
rateur sera limitée au volume de la cellule. D'autre part, $H_{1 z}$ apparait comme une sorte de moyenne sur la cellule de $H_{z}$, composante suivant $\mathrm{Oz}$ du champ magnétique $\overrightarrow{\mathrm{H}}$ régnant dans la cavité; nous-verrons plus précisément à l'appendice II que l'on a :

$$
n=\frac{\int_{\text {cellule }} H_{1 z} \cdot H_{z} d V}{\int_{\text {cavite }} H^{2} d V} \lesssim \frac{\left\langle H_{z}{ }^{2}\right\rangle_{\operatorname{cell}} V_{\operatorname{cell}}}{\left.<H^{2}\right\rangle_{\operatorname{cav}} V_{\text {cav }}} \lesssim 1
$$

La valeur de $n$ dépend du mode de la cavité considéré. Celui-ci sera choisi de manière à rendre $n$ maximum, mais devra surtout présenter une surtension $Q_{0} l_{a}$ plus élevée possible, malgré les perturbations apportées à la cavité (trous destinés au passage de la lumière de pompage, fonds démontables, etc.). Le mode TE 011 d'une cavité cylindrique et le mode correspondant d'une cavité sphérique répondent, comme nous le verrons, à ces deux conditions. 
En posant :

$$
\begin{aligned}
& 4 \pi \mu_{B}^{2} / K=K \\
& {\left[\hat{\sigma}_{++}^{(0)}-\hat{\sigma}_{--}^{(0)}\right]=\alpha} \\
& 1 / T_{2}=\delta \omega_{a}
\end{aligned}
$$

nous obtenons donc finalement :

$$
\frac{1}{Q_{a}}=-\frac{K \alpha n \eta}{\delta \omega_{a}+i\left(\omega-\omega_{a}\right)}
$$

Connaissant l'impédance d'entrée de la cavité, nous sommes dès lors ramenés à des calculs classiques en hyperfréquences. Par la ligne d'entrée, nous envoyons sur la cavité une onde incidente de puissance $P_{i}$; sur la même ligne se propage dans le sens apposé une onde réfléchie de puissance $P_{r}$, qu'un circulateur permet de séparer de l'onde incidente (fig. 2). Par le couplage de sortie s'échappe une onde transmise de puissance $\mathrm{P}_{\mathrm{S}}$. Le coefficient de réflexion (en puissance) à l'entrée est égal à :

$$
G_{r} \equiv \frac{P_{r}}{P_{i}}=\left|\frac{Z_{0}-Z_{i}}{Z_{0}+Z_{i}}\right|^{2}=\frac{\left[\frac{1}{Q_{0}}-\frac{1}{Q_{e e}}+\frac{1}{Q_{e s}}+\operatorname{Re} \frac{1}{Q_{a}}\right]^{2}+\left[2 \frac{\omega-\omega_{c}}{\omega_{c}}+\operatorname{Im} \frac{1}{Q_{a}}\right]^{2}}{\left[\frac{1}{Q_{0}}+\frac{1}{Q_{e e}}+\frac{1}{Q_{e s}}+\operatorname{Re} \frac{1}{Q_{a}}\right]^{2}+\left[2 \frac{\omega-\omega_{c}}{\omega_{c}}+\operatorname{Im} \frac{1}{Q_{a}}\right]^{2}}
$$

La puissance $P_{C}$ pénétrant (algébriquement) dans la cavité vaut $P_{i}-P_{r}=$ $P_{i}\left(1-G_{r}\right.$ ). Elle esst dépensée partie dans la cavité partie à travers le couplage de sortie, ce qui nous permet de définir un gain en transmission $G_{S}$ :

$$
G_{s} \equiv \frac{P_{s}}{P_{i}}=\frac{P_{c}}{P_{i}} \frac{\frac{1}{Q_{\text {es }}}}{\frac{1}{Q_{0}}+\frac{1}{Q_{\text {es }}}+\operatorname{Re} \frac{1}{Q_{a}}}=\left(1-G_{r}\right) \frac{\frac{1}{Q_{\text {es }}}}{\frac{1}{Q_{0}}+\frac{1}{Q_{\text {es }}}+\operatorname{Re} \frac{1}{Q_{a}}}
$$

soit encore : 
$(17-2)$

$$
G_{s}=\frac{\frac{4}{Q_{e e} Q_{e s}}}{\left[\frac{1}{Q_{0}}+\frac{1}{Q_{e e}}+\frac{1}{Q_{e s}}+\operatorname{Re} \frac{1}{Q_{a}}\right]^{2}+\left[2 \frac{\omega-\omega_{c}}{\omega_{c}}+\operatorname{Im} \frac{1}{Q_{a}}\right]^{2}}
$$

La largeur de la résonance atomique et la largeur de bande de la cavité sont faibles, de sorte que $\delta \omega_{a} / \omega_{a} \ll 1$ et $\delta \omega_{c} / \omega_{c} \ll 1$. Nous allons continuer les calculs en supposant $\delta \omega_{a} \ll \delta \omega_{C}$. Cette hypothèse est très bien vérifiée dans notre cas: nous verrons plus 1 loin que pour ${ }^{85} \mathrm{Rb}$, on a $2 \delta \omega_{a} / 2 \pi \simeq 60 \mathrm{~Hz}$ et $2 \delta \omega_{c} / 2 \pi \simeq 75 \mathrm{kHz}$. (Notons que, dans la théorie des lasers à gaz, on suppose au contraire $\delta \omega_{a}>>\omega_{c}{ }^{\prime}$ ) Par ailleurs, nous nous intéressons évidemment surtout aux variations de $G_{\Gamma}$ et $G_{S}$ directement liées à l'interaction atomes-cavité. Celle-ci se produira pour des fréquences très proches de la fréquence atomique $\omega_{a}$, sur un intervalle de l'ardre de $\delta \omega_{a}$ dans lequel, suivant I'hypothèse ci-dessus, les propriétés de la cavité vide varient très peu. Ceci nous amène à essayer de séparer les variations liées au système atomique (termes en $\omega-\omega_{a}$ ) de celles liées à la cavité (termes en $\omega-\omega_{c}$ ). Notons enfin que nous n'avons encore rien supposé sur le signe de la différence de populations $\sigma_{++}^{(0)}-\sigma_{--}^{(0)}=\alpha$. Bien que toutes les formules que nous allons obtenir soient valables quel que soit le signe de $\alpha$, nous nous intéresserons au cas de $\alpha>0$ (inversion de population). En définissant alors un "paramètre d'amplification" p par la relation.:

$$
P=K \alpha \cap n Q_{0} / \delta \omega_{a}
$$

il vient, après un calcul laborieux :

$(18-1) \quad G_{r}=\left\{1-\frac{\frac{4 \beta_{e}\left(1+\beta_{s}\right)}{\left(1+\beta_{e}+\beta_{s}\right)^{2}}}{1+\left(\frac{\omega-\omega_{c}}{\delta \omega_{c}}\right)^{2}}\right\}\left\{1+A_{r} \frac{1}{1+\left(\frac{\omega_{-} \omega^{\prime}}{\delta \omega^{\prime}}\right)^{2}}+B_{r} \frac{\frac{\omega^{\prime}-\omega^{\prime}}{\delta \omega^{\prime}}}{1+\left(\frac{\omega^{\prime}-\omega^{\prime}}{\delta \omega^{\prime}}\right)^{2}}\right\}$ 


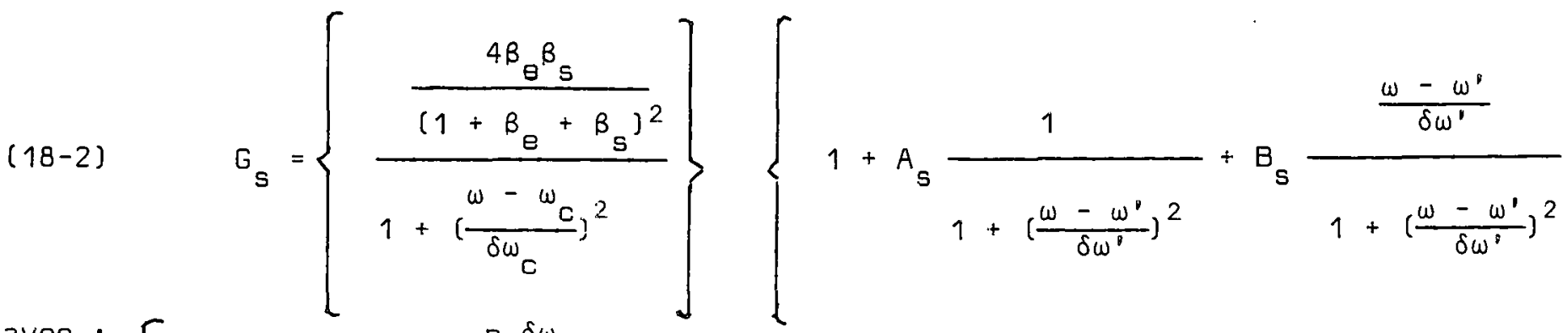

$$
\begin{aligned}
& \text { avec : }\left[\omega^{\prime}=\omega_{a}-\frac{\frac{p \delta \omega_{a}}{1+\beta_{e}+\beta_{s}}\left(\frac{\omega-\omega_{c}}{\delta \omega_{c}}\right)}{1+\left(\frac{\omega-\omega_{c}}{\delta \omega_{c}}\right)^{2}}\right. \\
& \delta \omega^{\prime}=\delta \omega_{a}\left[1-\frac{\frac{P}{1+B_{e}+\beta_{s}}}{1+\left[\frac{\left.\omega-\omega_{c}\right)^{2}}{\delta \omega_{c}}\right.}\right]
\end{aligned}
$$

Nous ne donnerons pas la forme des quatre coefficients $A_{r}, B_{r}, A_{s}, B_{s}$, qui est compliquée et sans intérêt. Il nous suffit de savoir qu'ils s'annulent avec $p$ et que. dans l'intervalle de fréquence envisagé ci-dessıs, ils peuvent être considérés comme des constantes ainsi d'ailleurs que $\left(\omega-\omega_{c}\right) / \delta \omega_{c}$. On reconnait alors dans $G_{r}$ et $G_{s}$ la présence d'un terme d'absorption (coefficient $A$ ) et d'ijn terme de dispersion (coefficient B) centrés sur la même fréquence $\omega^{\circ}$ et de même demi-largeur $\delta \omega^{\prime}$. Sans insister davantage sur ces formules générales, nous allons maintenant en examiner quelques applications intéressantes.

\section{B) APPLICATIONS}

\section{1) Condition d"oscillation}

Celie-ci a"obtient en forivant que $G_{r}$ et $G_{S}$ deviennent infinis, ce qui nécessite que $\omega=\omega^{\prime}$ et $\delta \omega^{\prime}=0$. Si, comme $c^{\prime e s t}$ toujours le cas en pratique, on ac- 
corde la cavité de telle façon que $\omega_{c} \approx \omega_{a}$, ces deux équations peuvent s'écrire :

$(19-1)$

$$
\frac{P}{1+\beta_{e}+\beta_{s}}=1 \quad \text { ou } \quad K \alpha \frac{n}{\delta \omega_{\exists}} n Q_{e}=1
$$

$(19-2)$

$$
\frac{\omega-\omega_{a}}{\omega-\omega_{c}}=-\frac{\delta \omega_{a}}{\delta \omega_{c}}
$$

La première de ces expressions nous montre la nécessité, pour obtenir l'oscillation. d'enfermer un grand nombre d'atomes soumis à un pompage efficace dans une cavité à surtension élevée tout en s'efforçant de maintenir un bon coefficient de remplissage et une largeur de raie la plus faible possible, conditions qui se révèleront plus loin très difficiles à remplir. La seconde nous permet de calculer 1. "entraînement de fréquence" de l'oscillation, ou plus généralement de la résonance, lorsque $\omega_{c}$ ne coincide pas avec $\omega_{a}$; on voit qu'il est d'autant plus marqué que la largeur atomique et la surtension de la cavité ont une valeur élevée. Par suite de cet effet, il sera nécessaire de contrôler avec soin l'accord de la cavité et de stabiliser sa température, comme nous le verrons au chapitre $V$.

\section{2) Cavité très désaccordée}

Si $\left(\omega_{a}-\omega_{c}\right) / \delta \omega_{c} \gg 1$, les expressions de $G_{r}$ et $G_{s}$ se réduisent à :

$(20-1)$

$$
G_{\Gamma} \simeq 1+
$$

$$
\frac{c^{t e}}{1+\left(\frac{w-w_{a}}{\delta w_{a}}\right)^{2}}
$$

$(20-2)$

$$
G_{S} \simeq \frac{c^{\text {te }}}{1+\left[\frac{\omega-\omega_{a}}{\delta \omega_{a}}\right)^{2}}
$$

On obtient donc une courbe d'absorption centrée sur $\omega_{a}$ et de demi-largeur égale à la demi-largeur atomique $\delta \omega_{a}$. Le temps de relaxation $T_{2}=1 / \delta \omega_{a}$ peut ainsi 
être déterminé expérimentalement et le résultat relié à ceux d'autres mesures effectuées sur le système atomique, telles que la mesure des divers temps de relaxation par voie optique dont nous avons parlé au chapitre précédent.

\section{3) Cavité accordée}

Nous allons étudier en détail le cas le plus intéressant où la cavité est exactement accordée sur la fréquence atomique : $\omega_{c}=\omega_{a}$. Compte tenu toujours de l'hypothèse $\delta \omega_{a} \ll \delta \omega_{C}, G_{\Gamma}$ et $G_{S}$ peuvent se mettre sous la forme :

(21-1)

$$
\begin{aligned}
& G_{I}=\left\{\begin{array}{c}
\frac{4 \beta_{e}\left(1+\beta_{s}\right)}{\left(1+\beta_{e}+\beta_{s}\right)^{2}} \\
\left.1-\frac{w-\omega_{a}}{2}\right)^{2}
\end{array}\right\}\left\{1+\frac{\left[\frac{1-\frac{p}{1-\beta_{e}+\beta_{s}}}{1-\frac{p}{1+\beta_{e}+\beta_{s}}}\right]^{2}-1}{1+\left[\frac{\omega-\omega_{a}}{\delta \omega_{a}\left(1-\frac{p}{1+\beta_{e}+\beta_{s}}\right)}\right]^{2}}\right\} \\
& G_{s}=\left\{\frac{\frac{4 \beta_{e} \beta_{s}}{\left(1+\beta_{e}+\beta_{s}\right)^{2}}}{1+\left(\frac{\omega-\omega_{a}}{\delta \omega_{c}}\right)^{2}}\right\}\left\{1+\frac{\left[\frac{1}{\left.1-\frac{p}{1+\beta_{e}+\beta_{s}}\right]^{2}}\right]^{2}}{1+\left[\frac{\omega-\omega_{a}}{\delta \omega_{a}\left(1-\frac{p}{1+\beta_{e}+\beta_{s}}\right)}\right]^{-1}}\right\}
\end{aligned}
$$

$(21-2)$

Dans ces deux expressions. le premier terme représente la variation banale due à la cavité vide. Celle-ci réfléchit totalement une fréquence éloignée $\left(G_{r}=1, G_{s}=0\right)$. Lorsque la fréquence excitatrice s'approche de $\omega_{a}=\omega_{c}$ ' l'absorption et la transmission de puissance suivent une courbe lorentzienne de demi-largeur $\delta \omega_{c}=\omega_{c} / 2 Q_{c}$; le gain par réflexion passe par un minimum égal à $\left(1-\beta_{e}+\beta_{s}\right)^{2} /\left(1+\beta_{e}+\beta_{s}\right)^{2}$ et le gain par transmission par un maximum égal à $4 \beta_{e} \beta_{s} /\left(1+\beta_{e}+\beta_{s}\right)^{2}$. 


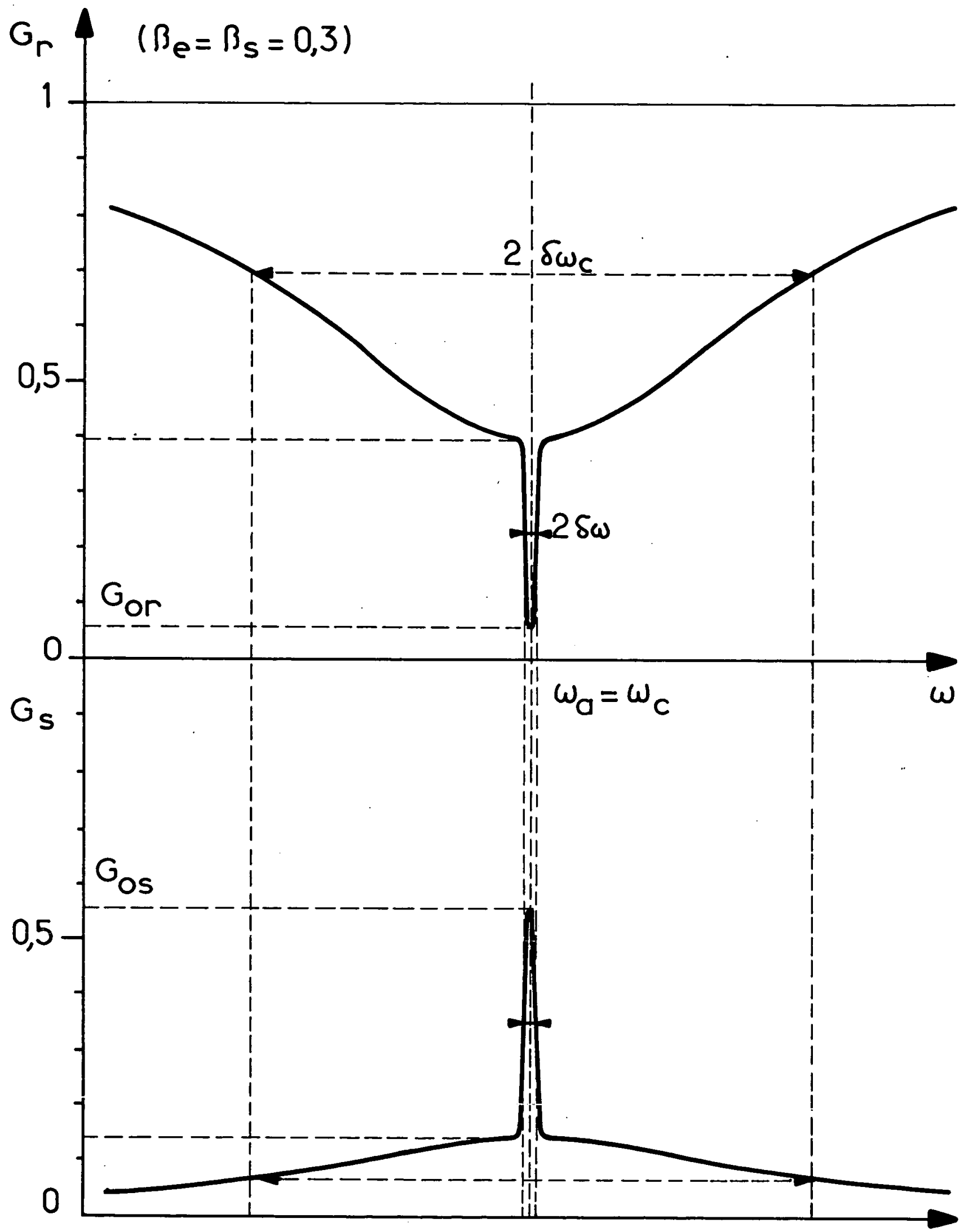

Fig. 4 


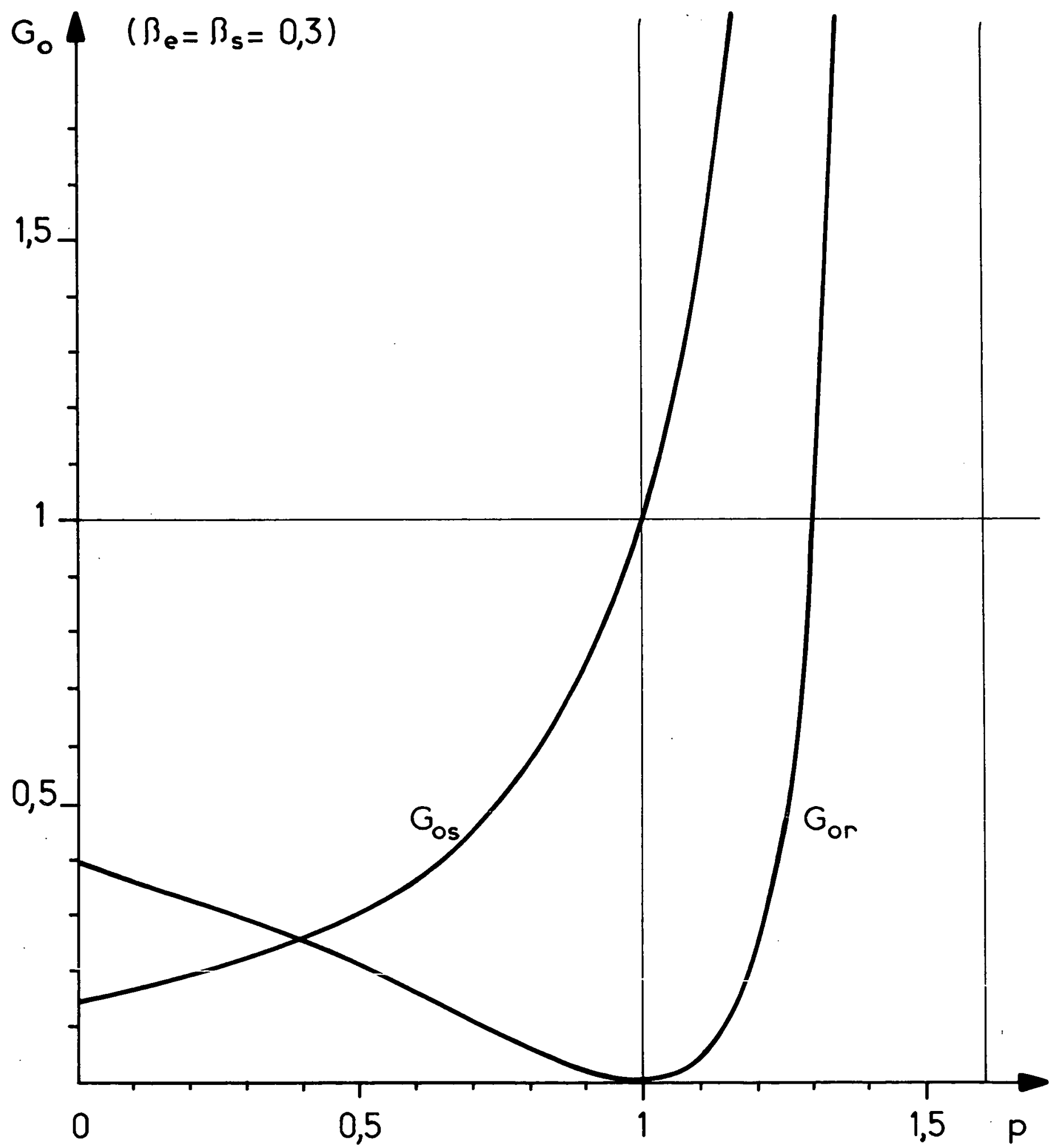

Fig. 5 


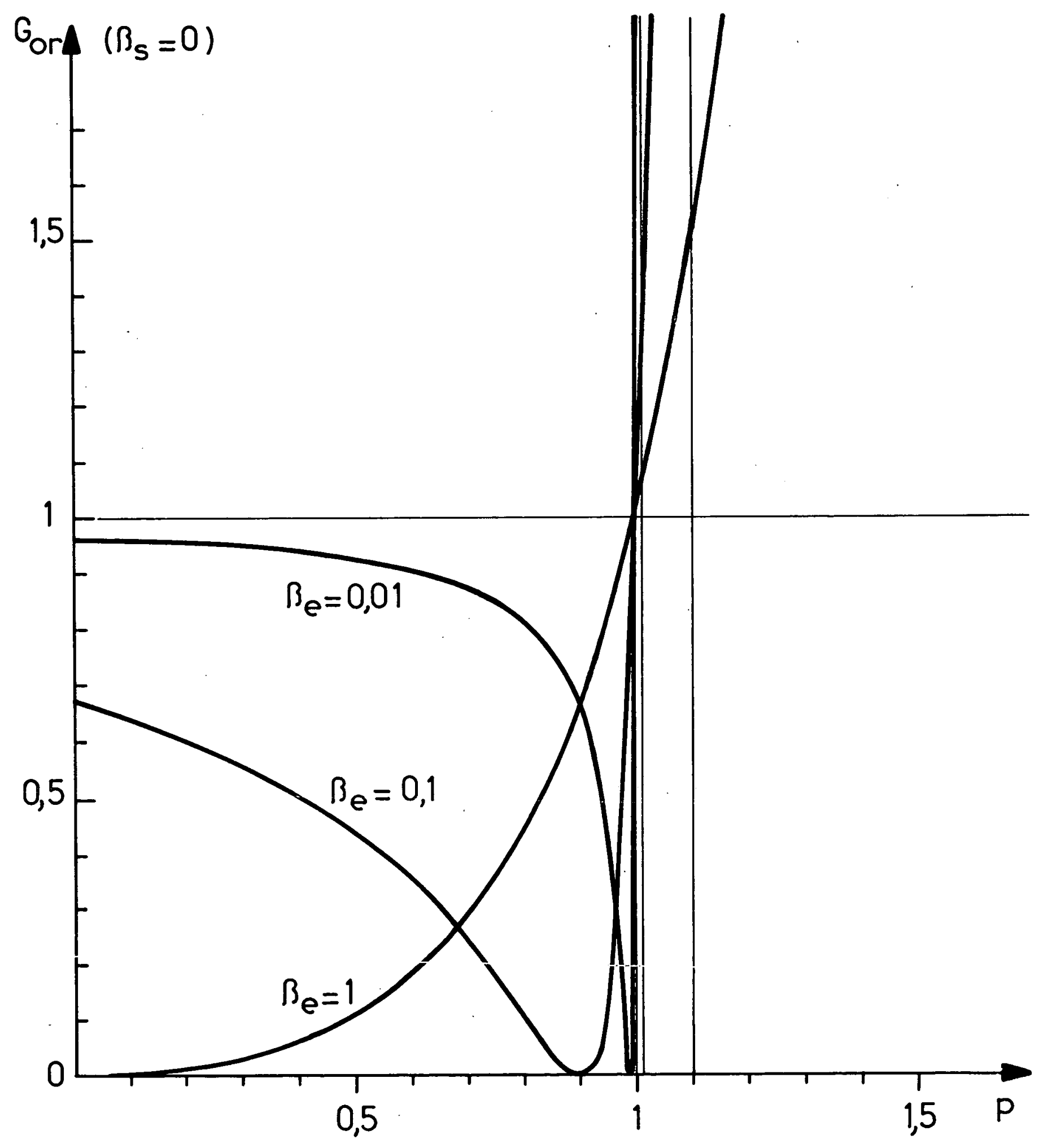

Fig. 6 

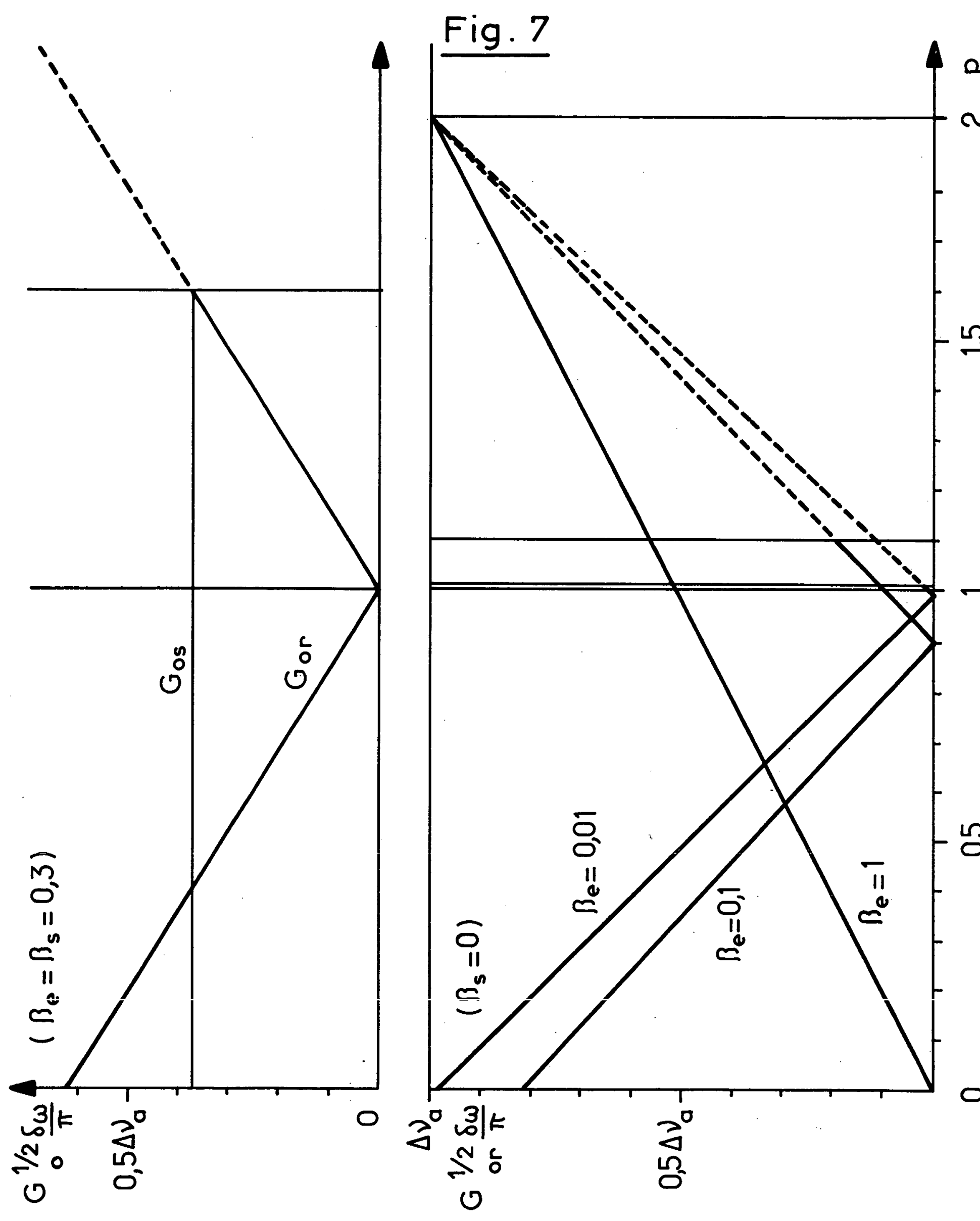
Le deuxième terme nous intéresse évidemment davantage. Il représente également une courbe d'absarption centrée sur $\omega_{a}$, mais de demi-largeur :

$$
\delta \omega=\delta \omega_{a}\left(1-\frac{p}{1+\beta_{e}+\beta_{s}}\right)
$$

Nous avons vu que la condition d'accrochage s'écrit $p /\left(1+\beta_{e}+\beta_{s}\right)=1$. L'équation ci-dessus illustre le rétrécissement par réaction de la raie atomique, do à l'amplification : la demi-largeur de raie, égale à $\delta \omega_{a}$ lorsque le paramètre d'amplification est petit, diminue quand $p$ augmente pour finalement s'annuler au seuil d'accrochage. La figure 4 indique la variation d'ensemble de $G_{\Gamma}$ et $G_{S}$ avec la fréquence, dans le cas où $\beta_{e}=\beta_{s}=0,3$ et $p=0,8$.

Il est particulièrement important d'étudier en fonction de $p$ la variation des valeurs $G_{o r}$ et $G_{o s}$ des gains $G_{r}$ et $G_{s}$ quand $\omega=\omega_{c}=\omega_{a}$. Celles-ci s'écrivent :

$$
G_{o r}=\frac{\left(1-\beta_{e}+\beta_{s}-p\right)^{2}}{\left(1+\beta_{e}+\beta_{s}-p\right)^{2}}
$$

$$
G_{o s}=\frac{4 \beta_{e} \beta_{s}}{\left(1+\beta_{e}+\beta_{s}-p\right)^{2}}
$$

La figure 5 représente $G_{\text {or }}$ et $G_{\text {os }}$ en fonction de $p$, dans le cas où $\hat{\mathrm{p}}_{e}=\hat{\mathrm{p}}_{\mathrm{s}}=0,3$. Ces deux gains devionnent infinis au sel!i 1. d'accrochage $(p=1,6)$ mais, tandis que la variation de $G_{\text {os }}$ est monotone, $G_{\text {or }}$ commence par décroître quand $p$ augmente et $s^{\prime}$ annule pour $p=1$ (notons que les valeurs remarquables $G_{o r}=0$ et $G_{o s}=1$ pour $P=1$ ne sont obtenues que si $\beta_{e}=\beta_{s}$. Nous avons souvent f́té amenés, pour des raisons technologiques, à étudier des cavités ne possédant qu'un couplage, pour lesquelles $\beta_{s}=0$. La figure 6 
représente dans ce cas $G_{\text {or }}$ en fonction de p pour diverses valeurs du couplage d"entrée $\beta_{e}$. Le gain s'annule pour $p=1 /\left(1-\beta_{e}\right)$.

Pour terminer cette discussion des propriétés ampificicatrices du système, nous avons porté sur la figure 7 la variation en fonction de p des deux quantités :

$$
G_{o r}^{1 / 2} \frac{\delta \omega}{\pi}=\frac{\delta \omega_{a}}{\pi} \frac{\left|1-\beta_{e}+\beta_{s}-p\right|}{1+\beta_{e}+\beta_{s}}
$$

$$
\sigma_{0 s}^{1 / 2} \frac{\delta \omega}{\pi}=\frac{\delta \omega}{\pi} \quad \frac{2 \sqrt{\beta_{e} \beta_{s}}}{1+\beta_{e}+\beta_{s}}
$$

qui définissent un facteur de mérite de l'appareil lorsque le gain est assez grand. Nous voyons qu'elles sont inférieures à $\Delta v_{a} \approx 60 \mathrm{~Hz}$ : au point de vue pratique, le système n'a donc que très peu d'intérêt en tant qu'amplificateur, et c'est son emploi en oscillateur qui justifiera toute cette étude.

\section{4) Amplitude du champ oscillant $\vec{H}$}

Revenons au cas général où la cavité n’est pas nécessairement accordée. Nous remarquons que. gräce à l'emploi des formules (16). nous avons pu déterminer toutes les propriétés radioblectriques de l'ensemble sans avoir calculé explicitement l'expression du champ $\vec{H}$. La valeur moyenne du carré de l'amplitude de celui-ci est cependant intéressante et assez facile à obtenir; en appliquant la relation (15) au cas de la boucle de sortie, il vient:

$$
<H^{2}>_{\text {cav }}=\frac{8 \pi Q_{e s}}{\omega V_{\text {cav }}} P_{s}
$$

Par ailleurs $P_{s}=G_{S} P_{i}$, en explicitant la formule (18-2), nous obtenons finalement : 
(25)

$<H^{2}>_{\operatorname{cav}}=\frac{32 \pi}{\omega V_{\operatorname{cav}}} P_{i}\left\{\frac{\frac{1}{Q_{e e}}}{\frac{1}{Q_{0}{ }^{2}\left(1+\beta_{e}+\beta_{s}\right)^{2}\left[1+\left(\frac{\omega-\omega_{c}}{\delta \omega_{c}}\right)^{2}\right]}}\right.$

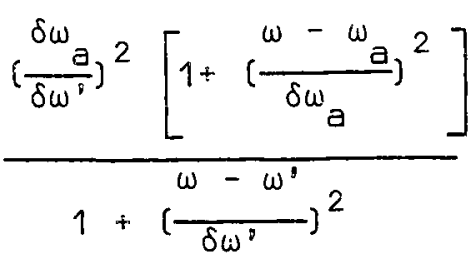

soit, dans le cas où $\omega_{c}=\omega_{a}$ :

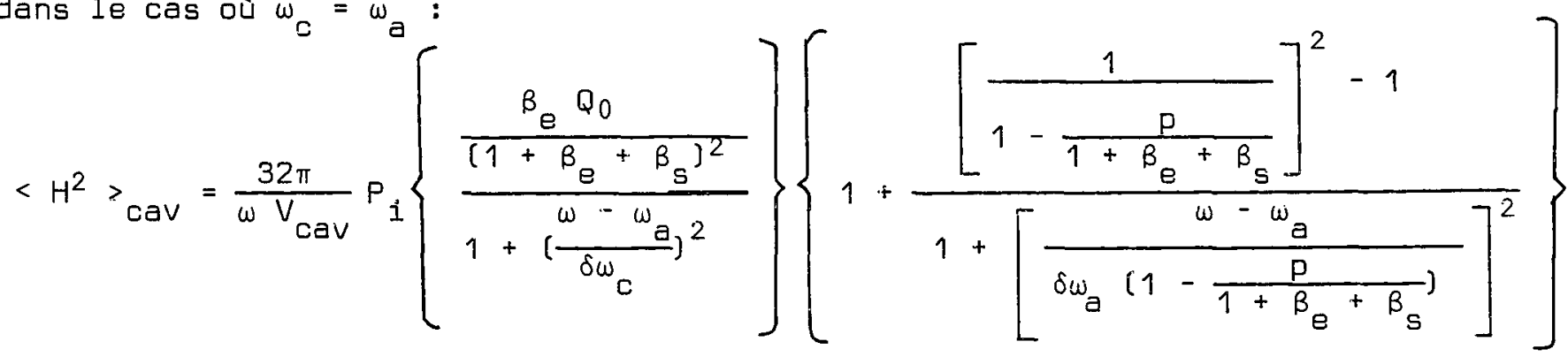

Le premier terme représente la variation, en fonction de la fréquence, de la densité d'énergie régnant dans la cavité vide. Le second illustre la réaction du système atomique sur le champ appliqué.

Si $\omega=\omega_{c}=\omega_{a}$, la formule se réduit à :

$$
<H^{2} i_{\operatorname{cav}}=\frac{32 \pi}{\omega_{a} V_{c a v}} P_{i} \frac{\beta_{e} Q_{0}}{\left(1+\beta_{e}+\beta_{s}-p\right)^{2}}
$$

A puissance incidente constante, l'amplitude du champ électromagnétique $\vec{H}$ régnant dans la cavité croit donc avec p et devient infinie au seuil d'accrochage. Tous les calculs précédents ayant été menés dans l'hypothèse de non saturation a c’est-à-dire de $\vec{H}$ faible, on peut s'interroger sur leur domaine de validité. Sans connaitre le détail de tous les processus vu relaxation ni préciser le rappnrt entre $H_{i z}$ et $\vec{H}_{\text {, nous }}$ pouvons nous inspirer des résultats valables pour les systèmes à deux niveaux et penser qu'une valeur raisonnable de $l^{\circ}$ amplitude de $\vec{H}$ pour laquelle la saturation du système atomique commence à apparaitre est donnée par la relation :

$$
\frac{\mu_{B}^{2}}{k^{2}}<\cdot H_{z}^{2}{ }_{\operatorname{cell}} \cdot T_{2}^{2} \simeq \frac{\mu_{B}^{2}}{k^{2}}<H^{2}>_{\text {cav }} \frac{n V_{\text {cav }}}{V_{\text {cell }}} T_{2}{ }^{2} \simeq 1
$$


La condition de non saturation s’écrit alors :

$$
P_{i}<\frac{k^{2} \omega_{a} V_{c e l 1}}{32 \pi \mu_{B}^{2} n} \frac{\left(\delta \omega_{a}\right)^{2}\left(1+\beta_{e}+\beta_{s}-p\right)^{2}}{\beta_{e} Q_{0}}
$$

Nous verrons dans la partie expérimentale que cette limitation impose des puissances d'entrée très basses. Elle devient d'autant plus sévère que $I^{\circledR}$ on $s^{\circ} a p p r o c h e$ du seuil d'accrochage.

\section{5) Retour surla détection optique}

Nous avons vu au chapitre II (équation (13) ) que le signal S de détection optique de la résonance 0-0 était donné par :

$$
S=\Delta L_{A} \propto \frac{\mu_{B}{ }^{2}\left(\hat{\sigma}_{+i}^{(0)}-\hat{\sigma}_{--}{ }^{0}\right) H_{1 Z}{ }^{2}}{\left(\frac{1}{T_{2}}\right)^{2}+\left(\omega-\omega_{a}\right)^{2}}
$$

$H_{1 z}^{2}$ étant proportionnel à $<H^{2}>$ cav calculé ci-dessus, on peut donc écrire :

$$
S \propto P_{i}\left\{\frac{1}{1+\left(\frac{\omega-\omega_{c}}{\delta \omega_{c}}\right)^{2}}\right\}\left\{\frac{1}{1+\left(\frac{\nu-\omega^{0}}{\delta \omega^{\prime}}\right)^{2}}\right\}
$$

Nous obtenons ainsi le résultat important suivant lequel la forme de la résonance en détection optique est la même que celle de la partie absorption du signal radioélectrique. En particulier, la résonance est entraînée par le désaccord de la cavité et rétrécie par réaction de la même manière. Ceci appelle toutefois deux remarques :

- Ce résultat n’est, comme les précédents, valable que loin de la saturation.

- Il nécessite que les atomes "observés" par détection optique et détection radioélectrique soient les mêmes. ce qui peut ne pas être le cas dans certaines conditions comme nous le verrons plus loin. 
En conclusion de ce chapitre, nous pouvons remarquer que l'influence du système atomique sur les propriétés radioélectriques de l'ensemble est entièrement décrite au moyen d'un seul paramètre $1 / Q_{a}=-k \alpha_{n} \eta /\left(\delta \omega_{a}+i\left(\omega-\omega_{a}\right)\right)$. Cette particularité donne à la question une grande unité. Par contre, elle rend l'étude physique du système atomique par des mesures d'amplification plus incertaine quecelle qui met à profit l'absorption optique en l'absence de radiafréquence; il est difficile de séparer l'influence relative de chacune des observables intervenant dans l'expression de $1 / Q_{a}$ (ou du paramètre d'amplification $p$ ), dont la valeur n'est par ailleurs obtenue qu'au prix de mesures délicates. En fait, les renseignements les plus intéressants obtenus sur le système atomique par excitation de la transition 0-0 sont la fréquence de résonance $\omega_{a}$ et le temps de relaxation $T_{2}$ de l'élément non diagonal $\sigma_{+-}$. Leur détermination à partir de la fréquence centrale et de la largeur des signaux de détection optique ou radioélectrique nécessitera d'ailleurs certaines vérifications :

- Détermination de $\omega_{a}$ : absence d'entraînement de fréquence (accord soigné de la cavité) ;

- Détermination de $\delta \omega_{a}=1 / T_{2}$ : absence de saturation (amplitude de $\vec{H}$ faible) et absence de rétrécissement par réaction : ce dernier résultat peut être obtenu, comme nous l'avons vu, en désaccordant fortement la cavité. Remarquons à ce propos que, toutes choses égales d'ailleurs, les trois effets ci-dessus sont d'autant plus marqués que la surtension de la cavité est plus élevée.

Notons enfin qu'au lieu d'établir les relations donnant la valeur en régime permanent des puissances $P_{r}$ et $P_{S^{\prime}}$ c'est-à-dire du carré de l'amplitude des ondes réfléchie et transmise, nous aurions également pu étudier le déphasage de ces ondes, ou calculer la réponse transitoire de l'ensemble à une impulsion de radiofréquence.

$$
\ldots / \ldots
$$


Nous avons vu au chэpitre précédent que lorsquétaient satisfaites les conditions fondamentales (19), l'ensemble cellule-cavité entrait en oscillation. Cet accrochage est dû à la réaction sur le système atomique, par l'intermédiaire de la cavité, du champ de radiofréquence qu'il a lui-mème produit. Nous allons reconsidérer cette question d’un point de vue plus général, en supposant qu'il existe, en plus de cette réaction interne, une réaction entre les couplages d’entrée et de sortie extérieure à la cavité.

L'ensemble cellule-cavité étant en condition d’amplification avec $\omega_{c}=\omega_{a}$, déconnectons le générateur de la ligne d'entrée et connectons la ligne de sortie sur l'entrée à travers un amplificateur de gain global G' supposé indépendant de $1^{\circ}$ amplitude et de la fréquence (fig. 8). Cet amplificateur impose alors la condition $P_{i}=P_{S} / G_{0 S}=G^{0} P_{s}{ }^{\prime} \cdot l^{\prime}$ ensemble-sera susceptible d'osciller $\left(P_{s} \neq 0\right)$ si-la phase de la réinjection est convenable et si $G_{o s} G^{\prime} \geqslant 1$ soit, d'après $1^{\prime e x p r e s s i o n ~}$ de $G_{\text {os }}$ donnée par (23-2), si le paramètre p satisfait à la condi.tion, plus générale que $(19-1)$ :

$$
\frac{P}{1+\beta_{e}+\beta_{s}-2 \sqrt{G^{0} \beta_{e} \beta_{s}}} \geqslant 1
$$

Comme pour tout oscillateur, l'approximation linéaire utilisée jusquici ne nous permet pas de calculer le niveau d’oscillation en régime permanent lorsque la condition ci-dessus est remplie. Le gain G' étant supposé indépendant de l'amplitude, celle-ci ne peut être limitée que par la saturation du système atomique lorsque le champ oscillant $\vec{H}$ (et done $H_{1 z}$ ) croît. Nous avions écrit au chapitre précédent que 


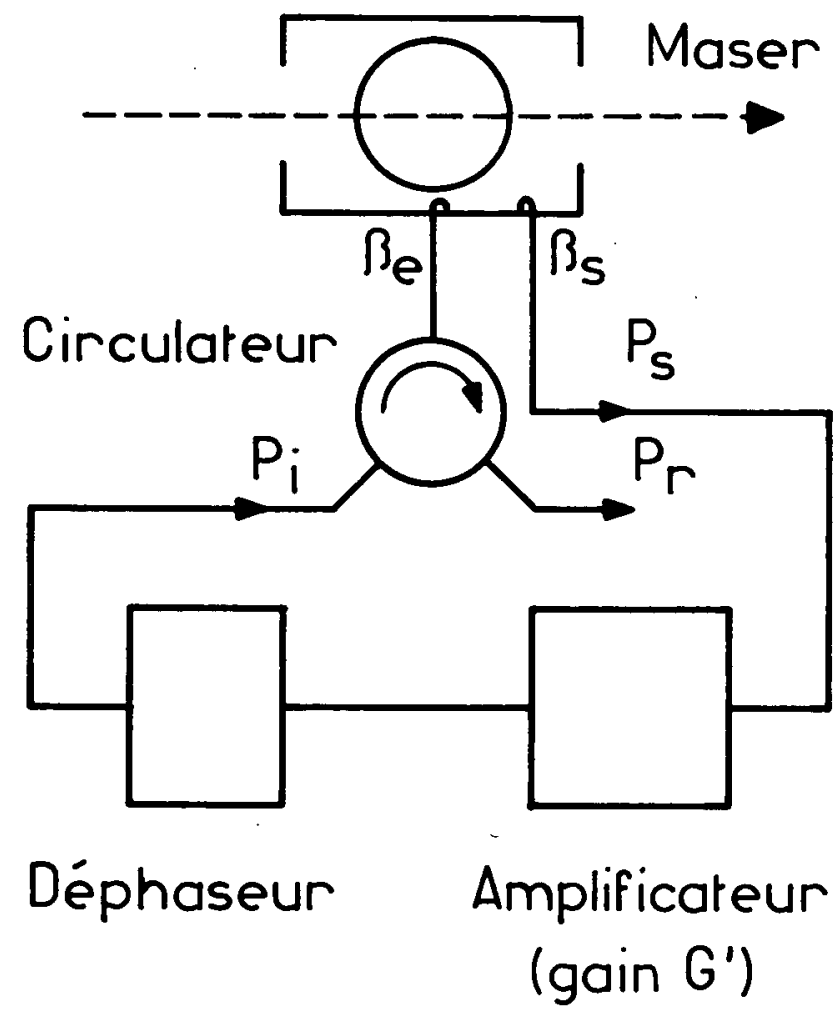

Fig. 8 
cette saturation apparaissait pour une valeur du champ donnée par $\mu_{B}{ }^{2}<H^{2}>_{\text {cav }} n V_{\text {cav }} T_{2}{ }^{2} / K^{2} V_{\text {cell }} \simeq 1$; d'une manière analogue, nous allons supposer ici que la partie réelle du facteur de surtension atomique $n^{D}$ est pas indépendante du champ régnant dans la cavité, mais obéit à une relation de la forme :

$$
\left(\operatorname{Re} \frac{1}{Q_{a}}\right)_{\text {sat }}=-\frac{K a n \eta}{\delta \omega_{a}\left(1+K<H^{2}>_{\text {Cav }}\right)}
$$

$\kappa$ étant un paramètre phénoménologique, voisin de $\mu_{B}{ }^{2} n V_{c a v} T_{2}{ }^{2} / K^{2} V_{c e l l}$. Les expressions $(23-1)$ et $(23-2)$ deviennent alors :

$(31-1)$

$$
\left(G_{\text {or }}\right)_{\text {sat }}=\left[\frac{1-\beta_{e}+\beta_{s}-\frac{P}{1+\kappa<H^{2}>_{\text {cav }}}}{1+\beta_{e}+\beta_{s}-\frac{P}{1+\kappa<H^{2}>_{\text {cav }}}}\right]^{2}
$$

$(31-2)$

$$
\left(G_{o s}\right)_{\text {sat }}=\frac{4 \beta_{e} \beta_{s}}{\left[1+\beta_{e}+\beta_{s}-\frac{p}{1+k<H^{2}>_{\text {cav }}}\right]^{2}}
$$

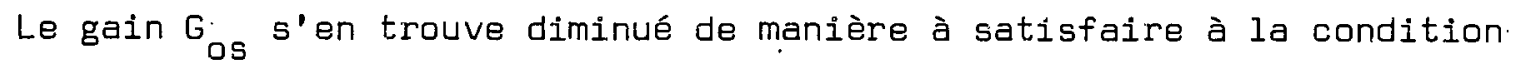
d'équilibre $\left(G_{o s}{ }^{\text {sat }} G^{\prime}=1\right.$, ce qui donne :

$$
\frac{P}{1+\beta_{e}+\beta_{s}-2 \sqrt{G^{\prime} \beta_{e} \beta_{s}}}=1+k<H^{2}>_{\mathrm{cav}}
$$

La condition d'équilibre fixe donc maintenant la valeur du champ dans la cavité. La relation $P_{S}=\omega<H^{2}>_{\text {cav }} V_{\text {cav }} / 8 \pi Q_{e s}$ nous permet alors, par une démarche inverse de celle que nous avions suivie pour calculer $\left\langle\mathrm{H}^{2}>\right.$ cav dans les conditions d'amplification linéaire, de calculer l'expression de $P_{S}$; celle de $P_{r}$ s'en déduit par application des formules du chapitre précédent. On obtient : 
$(32-1)$

$$
P_{r}=\frac{\omega_{a} V_{\text {cav }}}{8 \pi k} \frac{\left(\sqrt{G^{\prime} \beta_{s}}-\sqrt{\beta_{e}}\right)^{2}}{Q_{0}}\left[\frac{P}{1+\beta_{e}+\beta_{s}-2 \sqrt{G^{\prime} \beta_{e} \beta_{s}}}-1\right]
$$

$$
P_{s}=\frac{\omega_{a} V_{\text {cav }}}{8 \pi k} \frac{\beta_{s}}{Q_{0}}\left[\frac{p}{1+\beta_{e}+\beta_{s}-2 \sqrt{G^{0} \beta_{e} \beta_{s}}}-1\right]
$$

Le niveau d'oscillation en deux points de la boucle de réaction est ainsi déterminé; en l'absence de réaction extérieure, il suffit de poser $G^{\prime}=0$, La puissance émise est nulle au seuil d'accrochage, quand $p /\left(1+\beta_{e}+\beta_{s}-2 \sqrt{G^{\prime} \beta_{e} \beta_{s}}\right)=1$, et croit avec le paramètre d'amplification p. Si celui-ci est assez grand et $s^{\prime} G^{\prime}=0$, on peut écrire, en prenant $k \simeq \mu_{\mathrm{B}}{ }^{2} \eta \mathrm{V}_{\mathrm{cav}} \mathrm{T}_{2}{ }^{2} / \mathrm{K}^{2} \mathrm{~V}_{\mathrm{cell}}$ :

$$
\begin{aligned}
& P_{r} \simeq \frac{1}{2} \frac{\alpha n h v_{a} V_{c e l 1}}{T_{2}} \frac{\beta_{e}}{1+\beta_{e}+\beta_{s}} \\
& P_{s} \simeq \frac{1}{2} \frac{\alpha n h \nu_{a} V_{c e 11}}{T_{2}} \frac{\beta_{s}}{1+\beta_{e}+\beta_{s}}
\end{aligned}
$$

formules qu'il eût été facile d'établir directement. La puissance émise croît également avec $G^{\prime}$ et tend vers l'infini lorsque $\left(1+\beta_{e}+\beta_{s}-2 \sqrt{G^{\prime} \beta_{e} \beta_{s}}\right) \rightarrow 0$; en fait, lorsque le niveau d'oscillation devient élevé, l'hypothèse de la non saturation de l'amplificateur extérieur (G' indépendant de l'amplitude) n'est évidemment plus valable.

Des expressions semblables ont pu être établies dans d'autres cas, notam-

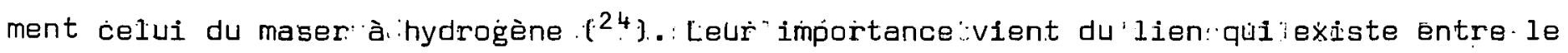
niveau de sortie et. la stabilité à court terme d'un oscillateur. Rappelons que les innstabilités cie fréquencé d'un oscillateur peuvent être classées en deux catégories :

a) les dérives, qui conduisent la fréquence à s'écarter lentement de sa valeur initiale. b) les fluctuations aléatoires de valeur moyenne nulle.

Les dérives sont difficilement calculables et sont dues à des causes qui dépendent de la constitution physique de l'oscillateur étudié. Deux effets de ce type, 
sur lesquels nous reviendrons, sont importants dans le cas du maser à vapeur alcaline : les déplacements de la fréquence atomique sous l'influence de la lumière de pompage et l'entraînement de fréquence dû à un désaccord de la cavité. Ces deux phénomènes affectent également la stabilité des horloges à pompage et détection optiques, mais peuvent beaucoup plus facilement y être minimisés, les différents paramètres n"ayant pas à satisfaire à la condition d'oscillation qui limite sévèrement les variations possibles : on peut par exemple combattre l'entraînement de fréquence [formule (19-2) du chapitre précédent) en employant une cavité de surtension $Q_{C}$ faible et dono de largeur de bande $\delta \omega_{c}$ importante, alors qu'une telie solution est inapplicable dans le cas du maser où $Q_{C}$ doit simultanément satisfaire à la condition (19-1). Le maser doit donc être affecté de dérives plutôt supérieures à celles de $I^{\natural}$ horloge et $I^{\prime}$ appareil ne pourra constituer un étalon de fréquence primaire.

Quelles que soient leurs causes, ces dérives sont cependant négligeables si le temps t' pendant lequel on observe la fréquence est inférieur à queïques secondes. Pour $t^{\prime}=1$ seconde ou molns, domaine de ce qu'il est corivenu d'appeler la stabilité à court terme, on n’a donc à envisager que les fluctuations aléatoires, qui se prêtent mieux au calcul. Soit $\Delta v$ la largeur de la résonance servant de base à l'oscil- . lateur : en écrivant que celui-ci n'est autre qu'un amplificateur du bruit thermique présent dans la bande de fréquences $\Delta \nu$, on démontre alors la relation $(25)$ :

$$
<\delta v^{2}>^{i} i 2=\Delta v \sqrt{\frac{k T}{2 P t}}
$$

où < $\delta v^{2}>^{1 / 2}$ est $1^{9}$ instabilité quadratique moyenne de la fréquence $v$ émise lorsque l'oscillateur maintenu à la température absolue $T$ et fournissant une puissance de sortie $P$ est observé durant un temps $t$ : : on voit qu'elle est d'autant plus faible que la puissance de sortie est plus grande. Dans le cas du maser à ${ }^{85} \mathrm{Rb}$, on peut prendre, comme nous ie verrons, $T \simeq 300^{\circ} \mathrm{K}, \Delta v_{a}=1 / \pi T_{2} \simeq 60 \mathrm{~Hz}$, et $P \simeq 0,05 \alpha$ nhv $v_{a} v_{\text {cell }} / T_{2} \simeq$ 
$10^{-10}$ watt:; valeur voisine de celle qui a été obtenue avec le maser à ${ }^{87} \mathrm{Rb}\left({ }^{4}\right)$; sur un temps $t^{\prime}=1$ seconde, nous obtenons alors $<\delta v^{2}>^{1 / 2} \simeq 2,7.10^{-4} \mathrm{~Hz}$ soit, pour une fréquence d'oscillation $v$ de $3 \mathrm{GHz}$, une instabilité relative $<v^{2}>^{1 / 2} / \nu \leqslant 10^{-13}$. Une telle valeur apparaît évidemment comme très intéressante. Une relation analogue, établie après une analyse plus poussée des différentes sources de bruit, a permis de comparer la stabilité théorique des masers à rubidium, à hydrogène et à ammoniac $(26)$. La figure 9 , extraite de la référence précédente, montre les résultats obtenus. On voit que le maser à rubidium devrait surpasser ses concurrents pour des temps d'observation voisins de $0.1 \mathrm{~s}$; pour les temps plus longs, la largeur plus faible de la transition 0-0 de l'hydrogène donne l'avantage au maser correspondant, tandis que sa puissance de sortie élevée assure au maser à ammoniac la meilleure stabilité pour des temps d'observation très courts. L'étude expérimentale correspondante n'en est actuellement qu'à ses débuts; par ailleurs, il conviendrait d'examiner également le cas d'un maser avec réaction extérieure ${ }^{27}$ ). Compte tenu des applications possibles que nous avons mentionnées brièvement dans l'introduction, l'ensemble constitue ainsi un sujet de recherches particulièrement intéressant. 


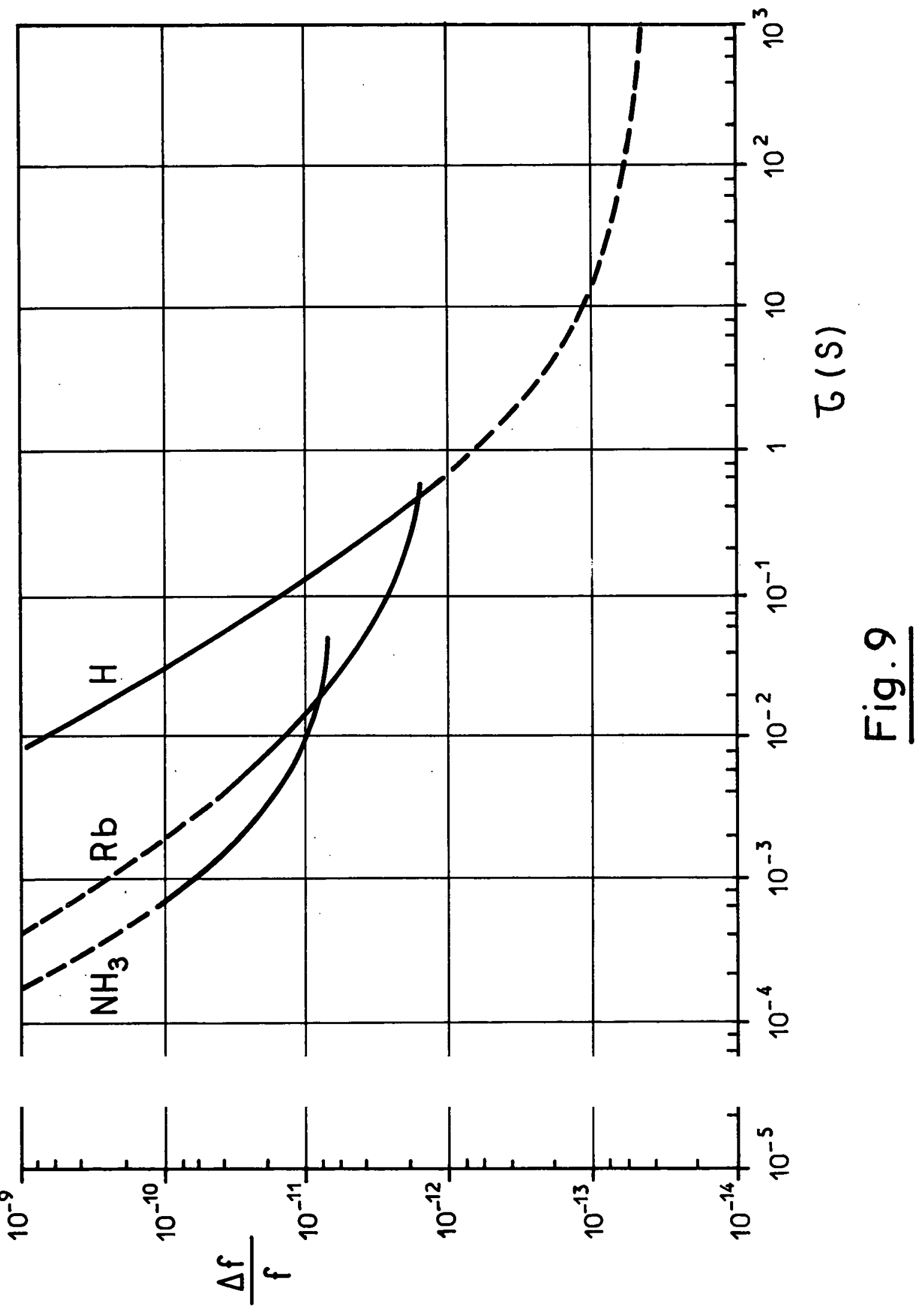




\section{APPLICATION AU CAS DU RUBIDIUM 85 : TECHNIQUES EXPERIMENTALES} 000000000000000000000050000000000000000000020000000000000000000

Nous allons maintenant exposer quelques applications et vérifications expérimentales de la théorie établie dans les chapitres précédents. Nous examinerons d'abord les conditions de pompage optique qui nous ont conduits à fixer notre choix sur ${ }^{85} \mathrm{Rb}$. Le montage expérimental fera ensuite l'objet d'une description détaillée, et plus spécialement l'ensemble cellule-cavité qui en est la partie essentielle. Les résultats obtenus et leur interprétation seront exposés dans le chapitre suivant.

\section{A) POMPAGE OPTIQUE DU RUBIDIUM 85}

Au moment où nous avons commencé notre travail, des recherches étaient déjà en cours depuis plusieurs années dans d'autres laboratoires en vue d'obtenir l'oscillation maser sur la transition $0-0$ de ${ }^{87} \mathrm{Rb}\left(\nu_{a}=6834,7 \mathrm{MHz}\right)$. Il était souhaitable de tenter l'expérience sur un autre atome : nous avons été conduits à choisir pour notre étude le rubidium $85\left(\nu_{a}=3035,7 \mathrm{MHz}\right.$ ), principalement à la suite de considérations sur le pompage optique que nous allons détailler maintenant.

Nous avons vu au chapitre II la condition à remplir pour obtenir une forte inversion de populations entre les sous-niveaux $\mid F_{+}$, $0>$ et $\mid F_{-}, 0>$ bordant la transition 0-0 : il faut que les deux composantes $B$ et $D$ de la raie de résonance optique émise par la lampe aient une intensité très supérieure à celle des composantes $A$ et $C$ (fig. 2). Dans le cas du rubidium, une coincidence accidentelle entre les composantes hyperfines de ${ }^{85} \mathrm{Rb}$ et ${ }^{87} \mathrm{Rb}$ nous permet $d$ 'obtenir ce résultat. La structure de la raie de résonance et la dénomination habituelle des différentes composantes hyper- 
fines pour les deux isotopes est donnée sur la figure $10{ }^{28}$ ). On voit que la composante $C$ de la raie $D_{1}$ de ${ }^{85}$ Rb coincide presque avec les composantes cd de la raie $D_{1}$ de ${ }^{87} \mathrm{Rb}$, et que la composante $A$ de la raie $D_{2}$ de ${ }^{85} \mathrm{Rb}$ coincide presque avec la composante a de la raie $\mathrm{D}_{2}$ de ${ }^{87} \mathrm{Rb}$. Dans les conditions habituelles, les lampes que nous utilisons émettent toutes les composantes hyperfines avec une intensité à peu près égale et ne peuvent donc produire le pompage hyperfin. Si par contre la lumière d'une lampe à ${ }^{85}$ Rb traverse un filtre rempli de vapeur de ${ }^{87} \mathrm{Rb}$, les composantes $C$ et $A$ seront en grande partie éliminées par absorption et la lumière ainsi filtrée répondra aux conditions d'un pompage hyperfin efficace. Le même mécanisme permet le pompage de ${ }^{87} \mathrm{Rb}$, en filtrant la lumière d'une lampe remplie de cet élément par un filtre à ${ }^{85} \mathrm{Rb}$. On améliore les coïncidences recherchées en introduisant dans le filtre un gaz étranger, ce qui a pour effet d'élargir et de déplacer les raies d'absorption (29). Dans un filtre à ${ }^{85} \mathrm{Rb}$ (pompage de ${ }^{87} \mathrm{Rb}$ ) il est habituel d'ajouter de l'argon sous une pression de quelques dizaines de torrs, déplaçant les composantes $C$ et $A$ vers les fréquences basses; pour un filtre à ${ }^{87} \mathrm{Rb}$ (pompage de ${ }^{85} \mathrm{Rb}$ ), on peut utiliser l'hélium, déplaçant les composantes cd et a vers les fréquences élevées.

Ce mode de pompage a été très employé dans le cas du rubidium $87\left({ }^{4}\right)\left({ }^{30}\right)$; nous 1'avons appliqué au rubidium 85. Nous verrons au chapitre suivant que la qualité du filtrage et l'inversion de populations atteintes dans ce dernier cas semblent inférieures à celles qui doivent être obtenues lors du pompage de ${ }^{87} \mathrm{Rb}$. L'explication doit sans doute en être recherchée dans la disposition relative des différentes composantes hyperfines. Malgré l'imprécision des mesures dont nous disposons (29), il semble en effet que l'élargissement des raies d'absorption du filtre dû au gaz étranger soit au moins égal à leur déplacement. Aux pressions de quelques dizaines de torrs nécessaires pour assurer une bonne coinncidence, la largeur des raies d'absorption doit 


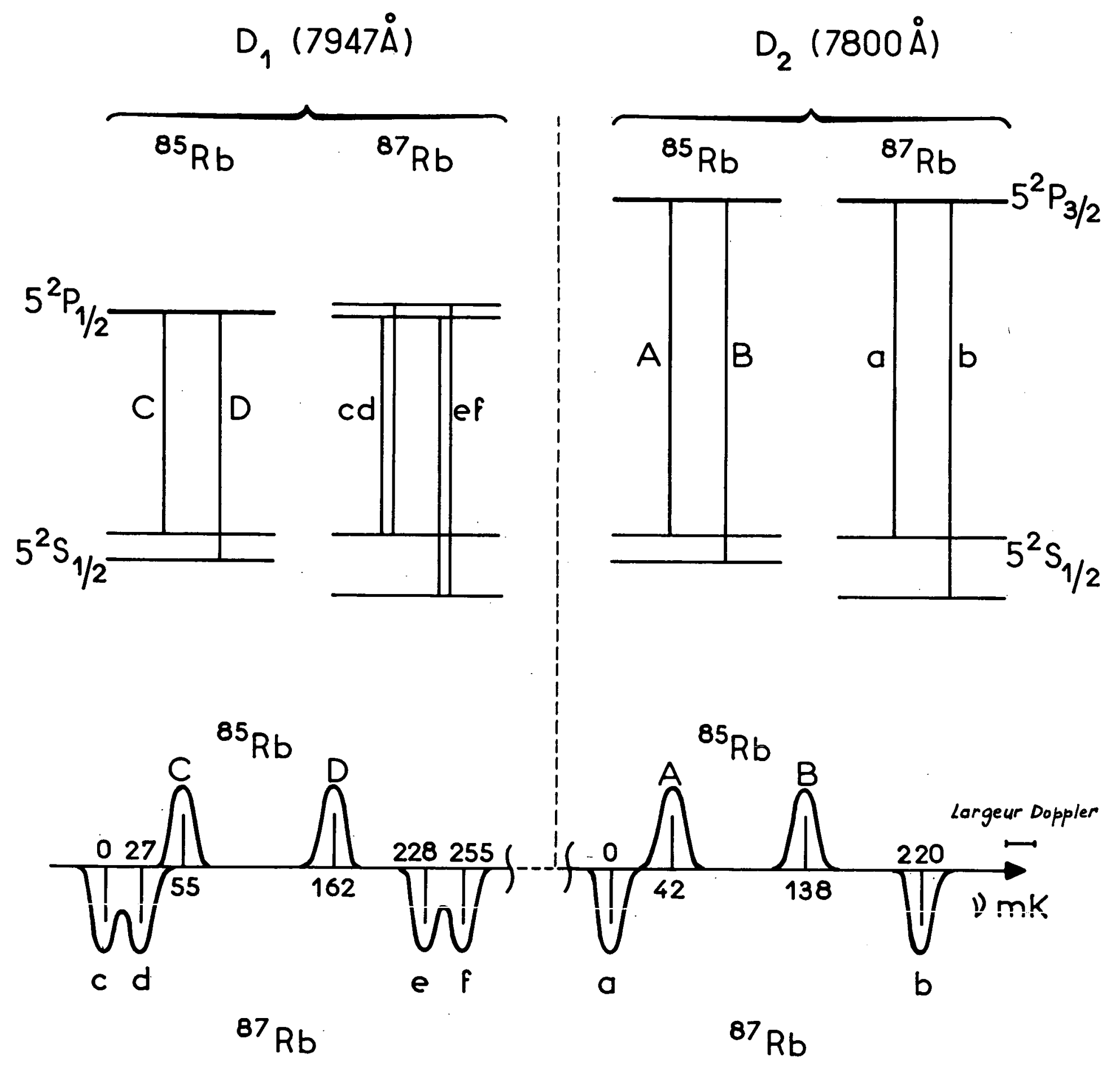

Fig. 10 
donc atteindre plusieurs largeurs Doppler. On voit alors sur la figure 10 que les ailes des raies d'absorption d'un filtre à ${ }^{87} \mathrm{Rb}$ ont tendance à absorber aussi les composantes utiles $B$ et $D$ émises par la lampe à ${ }^{85} \mathrm{Rb}$, tandis que l'inverse risque beaucoup moins de se produire, les composantes de ${ }^{85} \mathrm{Rb}$ se trouvant à l’intérieur de l'intervalle séparant les composantes de ${ }^{87} \mathrm{Rb}$. Le pompage hyperfin doit ainsi être plus efficace lorsqu'il est appliqué à ce dernier isotope.

L'absorption des photons appartenant aux composantes nuisibles par les atomes alcalins du filtre porte ces derniers dans l'état excité. Si la retombée subséquente vers l'état fondamental est radiative, la lumière de fluorescence contient à nouveau les mêmes composantes nuisibles, dont l'intensité "vue" par la cellule peut n'être pas négligeable dans le cas d'un système optique très ouvert. Il est donc intéressant d'ajouter dans le filtre quelques torrs d'azote, qui supprime par quenching toute retombée radiative. De même, l'isotope utiḷisé dans le filtre devra être d’une grande pureté, la présence de l'autre isotope, même en quantités assez faibles, pouvant, à température élevée, conduire à une absorption appréciable des composantes utiles.

On pourrait aussi envisager d'autres méthodes de pompage qui présentent l'avantage de ne pas être limitées au cas du rubidium :

a) Dans le cas d'une cellule remplie d'une forte tension de vapeur, la composante hyperfine $C$ est plus absorbée que $D$ et $A$ est plus absorbée que $B$, par suite du poids statistique supérieur du niveau $F_{+}$; la lumière issue de la lampe et ayant traversé les premiers centimètres de la cellule produit donc un pompage hyperfin dans la partie arrière de celle-ci. Ce procédé a permis la détection optique de la résonance 0-0 du sodium ( ${ }^{31}$ ), du potassium ( ${ }^{32}$ ), du rubidium (sans filtre) ( ${ }^{33}$ ) et du césium ( ${ }^{33}$ ); le calcul et l'expérience montrent cependant qu'il conduit à des inversions de popu- 
lations beaucoup moins élevées que dans le cas précédent..

b) On peut penser à réaliser un filtre hyperfin en appliquant, à une cellule contenant la vapeur de l'alcalin étudié, un champ électrique intense déplaçant les raies d'absorption par effet Stark $\left({ }^{34}\right)$.

c) On peut aussi rechercher une source dont la raie d’émission caincide avec les seules composantes utiles de la raie d'absorption de l'alcalin envisagé. Dans cet ordre d'idées, on a pu détecter optiquement la résonance $|4,0>\leftrightarrow| 3,0>$ du césium en employant comme source une lampe à argon dont les raies d'émission étaient déplacées par effet Zeeman ( ${ }^{35}$ ); le pompage utilisé conduisait toutefois à dépeupler le niveau supérieur $F_{+}=4$ et créait donc une répartition de populations inverse de celle qui nous intéresse ici. Dans le cas de cet alcalin, on peut songer par contre à employer comme source un laser à semi-conducteur du type Ga $A_{s_{1-x}} P_{x}$, dont la fréquence émise peut être déplacée en agissant sur la composition $x$ et sur la température de fonctionnement.

d) L'existence d'une différence d'intensité entre les composantes hyperfines n’est pas la seule possibilité pour créer l'inversion de populations souhaitée. On sait en effet que le pompage optique en lumière polarisée circulairement $\sigma_{+}$accumule les atomes dans le sous-niveau Zeeman $\mid F_{+}, \mu_{F_{+}}=F_{+}>$. La saturation des résonances Zeeman $\left(\Delta \mathrm{F}=0, \Delta \mu_{F}= \pm 1\right)$ de 1 'état $F_{+}$par un champ de radiofréquence (11), l'application de ce même champ en "impulsions $\pi / 2 "\left({ }^{36}\right)$, ou la modulation de la lumière de pompage à la fréquence Zeeman $v_{Z}\left({ }^{37}\right)$, permettent alors de transférer vers $1^{\circ}$ état $\mid F_{+}, 0>$ une partie de la population de l'état $\mid F_{+}, \mu_{F_{+}}=F_{+}>$. Malgré la perte de lumière due au polariseur (compensée d'ailleurs par l'absence de filtre hyperfin), ces méthodes paraissent susceptibles d'un bon rendement, mais elles présentent certaines difficultés techniques et, pour les deux dernières, l'inconvénient d'être discontinues. 
Rappelons enfin que les conditions de pompage optique sont différentes en présence et en l'absence de gaz étranger dans la cellule. Les études portant sur la résonance 0-0 des alcalins et. en particulier les nôtres, ont été le plus souvent effectuées en présence de quelques torrs d'un gaz étranger. Une telle pression n’élargit et ne déplace pas sensiblement la raie d"absorption optique; par contre, elle entraîne une désorientation totale de l'état excité, hypothèse dans laquelle nous avons mené les calculs du chapitre II. Tout comme dans le cas du filtre examiné plus haut, il est intéressant d'éliminer la lumière de fluorescence, dont la diffusion multiple réduirait l'inversion de populations atteinte; nous avons par suite utilisé comme gaz tampon l'azote, qui supprime cette fluorescence et dont les collisions avec les atomes alcalins dans leur état fondamental ne conduisent pas pour ces derniers à une relaxation trop énergique $\left({ }^{4}\right)\left({ }^{38}\right)$.

\section{B) DESCRIPTION DU MONTAGE EXPERIMENTAL}

Le dispositif permettant l'étude de ${ }^{85} \mathrm{Rb}$ est d'une assez grande complexité, en particulier l'appareillage destiné à l'excitation et à la détection radioélectrique de la résonance 0-0 $\left(\nu_{a}=3035,7 \mathrm{MHz}\right)$. Nous allons en décrire successivement les différentes parties, schématisées sur la figure 11.

1) Ensemble cellule-cavité

Le centre du montage est constitué par l'ensemble cellule-cavité, dont nous examinerons plus loin la structure de manière détaillée. Vu de l'extérieur, celui-ci apparait comme une enceinte en cuivre ou en acier inoxydable, présentant la symétrie de révolution autour d'un axe horizontal. Deux fenêtres circulaires centrées sur l'axe sont destinées au passage de la lumière de pompage, tandis qu'une ou deux prises coaxiales fixées sur la paroi latérale permettent le couplage radioélec- 
trique de l'ensemble avec le reste de l'appareillage. Sur le côté est assujetti un système mécanique assurant l'accord de la cavité. L'énsemble est placé dans un four à doubles parois de cuivre séparées par un isolant thermique, muni de deux fenêtres en Pyrex face à celles de la cavité. Le four peut être chauffé jusqu'à $80^{\circ} \mathrm{C}$ par un courant d'air chaud; on règle ainsi la tension de vapeur du rubidium à la valeur désirée (de $10^{-7}$ à $10^{-5}$ torr ). La stabilisation de la température doit être assez soigneusement assurée. Considérons en effet une cavité construite en cuivre (coefficient de dilatation $1,7 \cdot 10^{-5} /{ }^{\circ} \mathrm{C}$ ) et initialement accordée : une augmentation de température égale à $0,1^{\circ} \mathrm{C}$ produit un accroissement relatif égal à $1,7.10^{-6}$ des dimensions de la cavité et une diminution égale de la fréquence d'accord, soit $5 \mathrm{kHz}$ sur $3 \mathrm{GHz}$. Si la cavité a une surtension $Q_{c}=4.10^{4}$ (largeur de bande $75 \mathrm{kHz}$ ), la formule (19-2) du chapitre III, relative à l'entraînement de fréquence, nous apprend alors qu'une résonance atomique de largeur $60 \mathrm{~Hz}$ verra sa fréquence centrale abaissée de $4 \mathrm{~Hz}$ par la variation thermique envisagée. La très grande inertie thermique de la cavité facilite heureusement la stabilisation de la température, qui est contrôlée en permanence par un thermocouple relié à un enregistreur: Une tige traversant la paroi du four et reliée au système d'accord de la cavité permet d'açtionner celui-ci de l'extérieur.

Le four est enfïn placé lui-même au centré diun triple jeu de bobines en position d'Helmholtz, créant un champ magnétique faible et homogène $\vec{H}_{0}$ suivant $I$ 'axe de la cavité; on simplifie le réglage en dirigeant celui-ci vers le Nord magnétique terrestre.

\section{2) Partie optique}

Les différents éléments du système optique se succèdent le long d’un axe confondu avec celui de la cavité.

La lumière de pompage (raies $D_{1}: \lambda=7947 \AA$ et $D_{2}: \lambda=7800 \AA$ ) est pro- 


\section{LEGENDE DE LA FIGURE 11 (au verso)}

Schéma du montage expérimental

L : lampe

$F$ : filtre

0 : obturateur

$\mathrm{H}_{0}$ : champ magnétique statique

c : cellule de résonance

$K$ : cavite

PM : photomultiplicateur

B.F. : oscillateur basse fréquence

D.S. : détection synchrone

o : oscilloscope

$P_{1}, P_{2}$ : pilotes à quartz

$X$ : multiplicateur

M. $M^{\prime}$ : mélangeurs

A : atténuateur

Fr : fréquencemètre

C : circulateur

$P_{i}$ : puissance incidente

$P_{r}$ : puissance réfléchie

$P_{\mathbf{S}}$ : puissance transmise

O.L. : oscillateur local

$\mathrm{R}$ : récepteur

Fréquences indiquées en $\mathrm{MHz}$ 


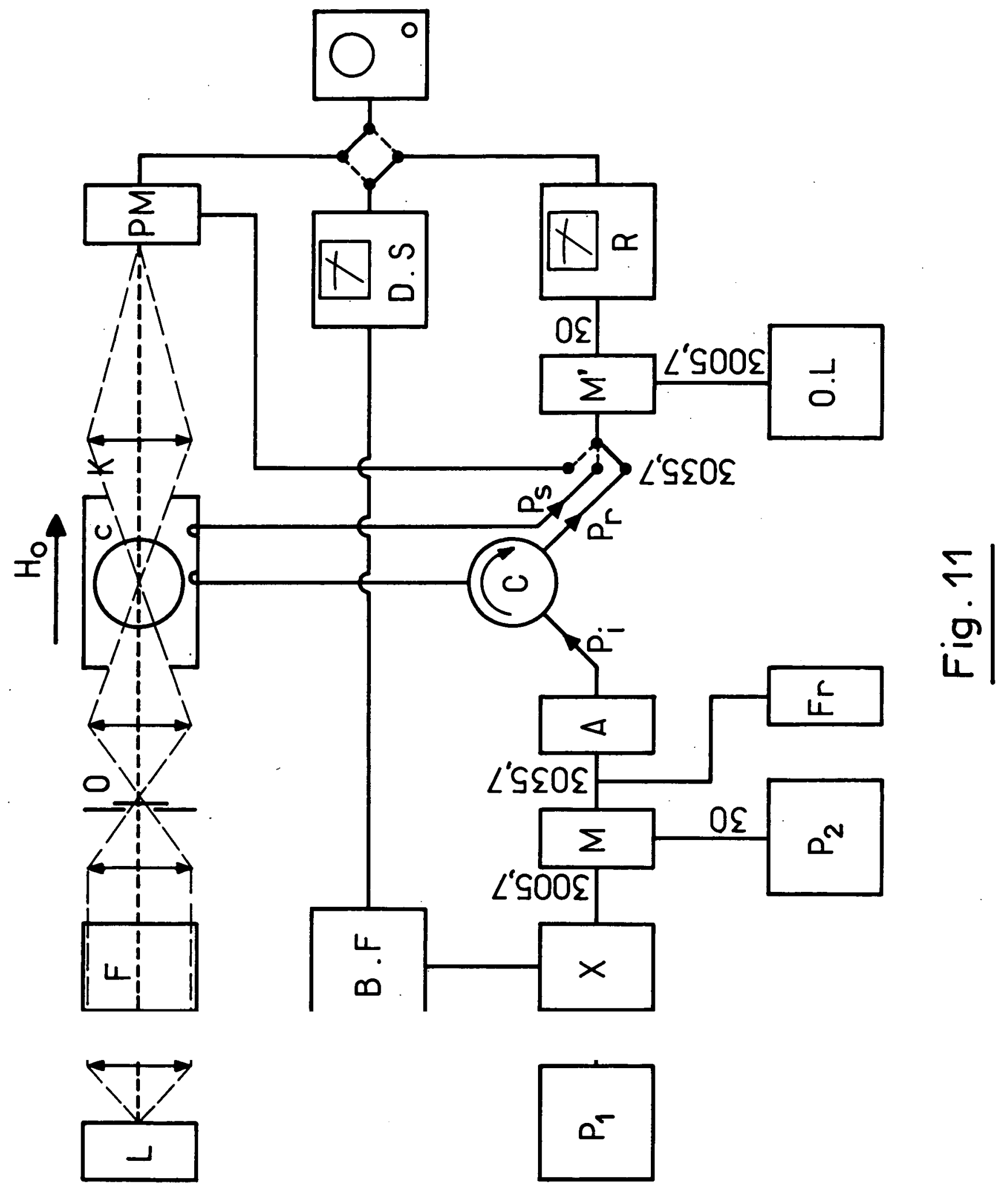


duite par une lampe sans électrodes en Pyrex. Celle-ci a la forme d'un disque de $50 \mathrm{~mm}$ de diamètre et $10 \mathrm{~mm}$ d'épaisseur, prolongé par un queusot latéral. Elle contient quelques milligrammes de ${ }^{85} \mathrm{Rb}$ et environ 1 torr d'argon nécessaire à l'amorçage. L'excitation est assurée par un oscillateur à magnétron, dont l'alimentation est très soigneusement stabilisée en courant; l'antenne placée près de la lampe émet environ 100. watts à 2450 MHz. Il est nécessaire d'amorcer la décharge avec un oscillateur Tesla. Le fonctionnement général et le profil des raies émises sont très sensibles au régime thermique des différents points de la lampe; un petit four tubulaire permet de régler la température du queusot, tandis que le disque est calorifugé par une enceinte en stratifié verre-silicone comportant deux fenêtres en Pyrex. Une fois le régime permanent établi, la lumière émise se compose presque uniquement de la raie de résonance de ${ }^{85} \mathrm{Rb}$, les quatre composantes $A, B, C, D$ ayant une intensité à peu près égale et s'étendant chacune sur un intervalle spectral de 2 à 3 largeurs Doppler. Le flux lumineux total recueilli dans le système optique qui suit la lampe $(7 \mathrm{~cm}$ de diamètre utile, ouvert à $f: 1)$ est d'environ $10^{17}$ photons par seconde. Il est constant à quelques $10^{-4}$ près sur une seconde et ne varie pas de plus de $10^{-2}$ d'un jour à I'autre.

Le faisceau ainsi formé est envoyé sur le filtre destiné, comme nous l'avons vu, à lui donner lá composition spectrale désirée. Ce filtre est constitué d'une cuve cylindrique en Pyrex de $80 \mathrm{~mm}$ de diamètre et $36 \mathrm{~mm}$ d'épaisseur, contenant quelques milligrammes de ${ }^{87} \mathrm{Rb}$ et quelques dizaines de torrs d'hélium ou d'un mélange héliumazote. La cuve est placée dans une enceinte calorifugée munie de deux fenêtres en Pyrex et chauffée électriquement vers $80^{\circ} \mathrm{C}$ par un câble "Thermocoax" court-circuité à une extrémité, ne créant pas de champ magnétique parasite. On peut appliquer contre les fenêtres des écrans de tulle noir atténuant le flux lumineux dans un rapport connu. 
A la sortie du filtre, le faisceau est refocalisé sur un obturateur de $25 \mathrm{~mm}$ d'ouverture, capable de couper ou rétablir la lumière en $1.5 \mathrm{~ms}$ environ; repris par une troisième lentille, il pénètre dans le four central et traverse l'ensemble cellule-cavité suivant son axe. La lumière transmise est enfin dirigée par une quatrième lentille sur le récepteur photosensible. Nous avons utilisé soit un photomultiplicateur infrarouge monté en cellule pour minimiser 1 "effet de fatigue ( ${ }^{39}$ ), soit une photopile au silicium débitant sur une faible impédance de manière à fournir un courant rigoureusement proportionnel au flux lumineux. La sortie du récepteur est connectée à l'entrée verticale d'un oscilloscope continu très sensible $(100 \mu \mathrm{V} / \mathrm{cm})(40)$ dont le balayage horizontal est proportionnel au temps, qui permet l'étude des exponentielles de pompage; on photographie l'écran avec un appareil à développement instantané. Dans quelques expériences, d'ailleurs négatives, nous avons utilisé comme récepteur un phototube hyperfréquence $(41)$.

Le montage ainsi décrit s'est révélé très bien adapté à l'étude des cellules par voie optique mais, dans les conditions donnant lieu à la meilleure amplification radioélectrique (tension de vapeur élevée dans la cellule) fournit au niveau de la cellule un flux lumineux trop faible. Nous avons alors été amenés à supprimer le récepteur photoélectrique et l'obturateur et à installer de chaque côté de la cellule un faisceau d'illumination le plus ouvert possible.

Les différents éléments du système optique et le four central sont vissés sur une plaque en duralumin de $12 \mathrm{~mm}$ d’épaisseur, elle-même fixée rigidement à une lourde table en bois de manière à éviter toute vibration. Des capots en aluminium noirci les protègent contre la lumière parasite et empêchent que le rayonnement hyperfréquence servant à exciter la lampe ne perturbe le reste du montage. 


\section{3) Générateur hyperfréquence}

La résonance atomique étudiée a une largeur $\Delta \nu_{a}$ de quelques dizaines de $\mathrm{Hz}$, Pour une fréquence $\nu_{a} \simeq 3035,7 \mathrm{MHz}$, ceci correspond à une largeur relative $\Delta \nu_{a} / \nu_{a} \simeq 10^{-8}$, valeur qui diminue encore considérablement au voisinage du seuil d'accrochage comme nous l'avons vu au chapitre III. La source hyperfréquence utilisée pour exciter la transition lorsque le système atomique est en-dessous des conditions d'oscillation doit done présenter des fluctuations relatives de fréquence au plus égales à quelques $10^{-10}$. Une telle stabilité, inaccessible aux oscillateurs hyperfréquences usuels, n’est atteinte que par des étalons à quartz spécialement construits et oscillant au vaisinage de $5 \mathrm{MHz}$. Le point de départ de la source que nous utilisons est un pilote $P_{1}$ de haute pureté spectrale oscillant à $5,218 \ldots$ MHz. Ce pilote attaque une chaîne de multiplication à varactors ( ${ }^{42}$ ) qui nous fournit l'harmonique 72 de la fréquence initiale, à $375,7 \mathrm{MHz}$. Elle est suivie d'un second multiplicateur, à varactor également, accordé sur l'harmonique 8 à $3005,7 \mathrm{MHz}$. Le bon rendement de cette seconde multiplication exige une adaptation très soignée des impédances à l'entrée et à la sortie, que nous avons obtenue. à l'aide d'éléments coaxiaux dont la stabilité mécanique pose quelques problèmes. On fait enfin battre, dans un mélangeur à cristal, le signal à 3005,7 MHz avec un signal à $30 \mathrm{MHz}$ issu d'un second pilote $P_{2}$; après filtrage par une cavité accordée, nous obtenons ainsi l'onde à $3035,7 \mathrm{MHz}$ désirée. Pour faire varier celle-ci d'une tròs petite quantité autour de la valeur $v_{a}$, il est commode d'agir sur le seul pilote $P_{2}$.

Un fréquencemètre, comportant un étage hyperfréquence très sensible $\left({ }^{4} 3\right)$, nous permet de contrôler la fréquence en divers points : dans la partie hyperfréquence, les coupleurs directionnels sont très précieux pour cet usage. La stabilité à long terme de la fréquence finale est égale à celle du quartz de départ, soit $10^{-9}$ par jour. 
La stabilité à court terme de celui-ci $\left(5.10^{-11} / \mathrm{s}\right.$ d'après le constructeur) se retrouve sur la fréquence finale sans paraitre affectée par les multiplications de fréquence successives (44); nous décrirons plus loin une expérience indiquant pour notre source une stabilité de $3.10^{-11}$ sur une seconde. La valeur absolue des fréquences en temps $A_{1}$ peut être obtenue à $5 \cdot 10^{-10}$ près par comparaison avec l'émission pilote de Droitwich à $200 \mathrm{kHz}(45)$; dans cette échelle. la fréquence 0-0 de l'atome de rubidium 85 non perturbé est égale à $3035732439 \pm 5 \mathrm{~Hz}(46)$.

Le niveau de sortie à $3035,7 \mathrm{MHz}$ est de 2 microwatts. La formule (28) du chapitre III, appliquée à un cas typique $\left(V_{\text {cell }} \simeq V_{\text {cav }} \simeq 1730 \mathrm{~cm}^{3}, \eta \simeq 1,2 \delta \omega_{a} / 2 \pi=60 \mathrm{~Hz}\right.$, $\beta_{e}=0,01, \beta_{s}=0, P=0,5, Q_{0}=4.10^{4}$ J nous impose une puissance à l'entrée de la cavité inférieure à $10^{-8}$ watt sous peine de saturation; nous avons travaillé le plus souvent avec des puissances comprises entre $10^{-9}$ et $10^{-11}$ watt. Un jeu d'atténuateurs calibrés permet de se ramener au niveau nécesseire et peut être utilisé pour les mesures de gain. La sortie de l'atténuateur est connectée à un circulateur, lui-même relié à la prise coaxiale d'entrée de la cavité et permettant d'isoler la puissance réfléchie par celle-ci.

\section{4) Récepteur hyperfréquence}

Les faibles signaux ci-dessus nécessitent l'emploi d'un récepteur très sensible. L'étage d'entrée de celui que nous utilisons (47) est un mélangeur-préamplificateur qui impose son facteur de bruit $(8,5$ db) à l'ensemble. Il peut être attaqué par trois signaux différents : la puissance réfléchie $P_{r}$, apparaissant à la porte 3 du circulateur, la puissance transmise $P_{S}$ apparaissant au couplage de sortie de la cavité, enfin éventuellement le signal issu d'un phototube hyperfréquence. Le mélangeur reçoit d'autre part une onde à $3005,7 \mathrm{MHz}$ de fort niveau (1 mW) provenant d'un oscillateur local. Bien que, comme nous l'avons vu, la chaîne de multiplication 
ait été conçue pour pouvoir fournir cette onde à 3005,7 MHz, nous avons presque exclusivement utilisé comme oscillateur local un générateur commercial à klystron dont la stabilité était suffisante dans la plupart des cas. Le battement à $30 \mathrm{MHz}$ ainsi produit est envoyé dans un récepteur à très grand gain (130 db) et à bande passante commutable (5 $\mathrm{MHz}$ ou $25 \mathrm{kHz}$ ); on recueille à la sortie soit le $30 \mathrm{MHz}$ amplifié, soit, après détection, le niveau "vidéo" sous plusieurs impédances, ques l'on peut également observer sur un voltmètre incorporé à l’appareil. Pour certaines expériences (détermination de la largeur et de la forme de raie), nous avons utilisé un détecteur synchrone : on attaque celui-ci par le niveau vidéo issu du récepteur (ou éventuellement, dans le cas d'une détection optique,par le courant du détecteur photoélectrique), tandis que 1'oscillateur BF de référence module à environ $10 \mathrm{~Hz}$ la fréquence excitatrice, par l'intermédiaire d'une "varicap" agissant sur l'un des étages de la première chaîne multiplicatrice. Le signal issu du détecteur synchrone est lu sur un voltmètre, ou peut être enregistré. Pour mesurer les puissances incidente, réfléchie et transmise, nous avons surtout utilisé l'indication $V$ du voltmètre incorporé au récepteur, la bande passante de celui-ci étant choisie égale à $5 \mathrm{MHz}$ : la sensibilité, conforme à la valeur théorique $\left(1,5.10^{-13}\right.$ watt) est en effet suffisante et la stabilité est meilleure qu'en bande étroite. La valeur de $V$ dépend de la puissance mesurée, mais aussi du réglage du gain sur le récepteur et de la loi de réponse du détecteur final. Nous avans étalonné celui-ci et l'avons trouvé sensiblement linéaire, c'est-à-dire que $V$ est sensiblement proportionnel à la racine carrée de la puissance reçue : lorsque les variations de celle-ci sont faibles, on peut d'ailleurs sans grande erreur les considérer comme proportionnelles aux variations correspondantes $\Delta V$. Lorsque nous désirions une plus grande précision, nous avons opéré par substitution en remplaçant le signal étudié par un signal à la même fréquence issu d'un générateur possédant un atténuateur de sortie très précis. On se ramène alors toujours à la même valeur de $V$ et on lit le rapport 
des puissances sur l'atténuateur.

\section{5) Oscillateur à réaction extérieure}

Pour l'étude de l'oscillation avec réaction extérieure, nous avons utilisé un amplificateur à diode tunnel de gain fixe 20 db et de facteur de bruit 5,5 db. Un atténuateur variable étalonné et un déphaseur complètent la boucle de réaction.

Tous les appareils précédents, constituant la partie radioélectrique du montage, sont soit installés sur des tables en bois, soit empilés dans des racks. Par crainte des champs magnétiques parasites, l'ensemble est à une certaine distance de la cavité, ce qui a imposé des câbles de raccordement assez longs (2 mètres). Ceux-ci sont réalisés en qualité antisignal (RG $213 \mathrm{U}-\mathrm{AS}$ ) afin de réduire les parasites électriques associés aux déformations mécaniques et sont montés en usine avec des connecteurs de type $N$ soigneusement vérifiés. Ces câbles ont cependant constitué dans nos expériences une cause certaine d'instabilité, les changements d'impédance liés à leur déformation n'étant pas toujours négligeables par rapport à ceux que nous cherchions à mesurer; le petit matériel coaxial (atténuateurs, adaptateurs d'impédance, coupleurs directionnels, filtres..) : est par contre d'un emploi très pratíque.

\section{C) STRUCTURE DE L'ENSEMBLE CELLULE-CAVITE}

Cet ensemble est la partie essentielle du montage, qui conditionne le reste de l'expérience. Il doit conduire à la plus grande valeur possible du paramètre d'amplification $p$, en vue d'atteindre finalement le seuil d'oscillation. En nous rappelant la condition établie au chapitre III (équation (19-1) :

$$
\frac{4 \pi \mu_{B}{ }^{2}}{h} \alpha \frac{n}{\delta \omega_{a}} n Q_{C}=1
$$


nous voyons que nous pouvons espérer obtenir l'oscillation avec $\alpha=6.10^{-2}$, $n=1,6 \cdot 10^{11}$ atomes $/ \mathrm{cm}^{3}, 2 \delta \omega_{a} / 2 \pi=\Delta \nu_{a}=60 \mathrm{~Hz}, \eta=0,5$ et $Q_{c}=4 \cdot 10^{4}$. Nous verrons par la suite que les valeurs précédentes sont des ordres de grandeur dont on ne peut guère s'écarter : il en résulte pour l'ensemble cellule-cavité de sévères exigences que nous allons passer en revue.

1) Choix du mode de résonance de la cavité

D'après l'expression ci-dessus, le mode employé doit conduire à une valeur maximum du produit $n Q_{C}$ (ou $n Q_{0}$ ). Dans une cavité cylindrique, ceci impose pratiquement le choix d'un mode TE omn. Leur surtension nominale est en effet élevée; de plus, les lignes de courant dans les parois, associées à ces modes, sont des cercles centrés sur l'axe de la cavité. La présence de trous dans les fonds, les discontinuités à la jonction des fonds et de la paroi latérale, et plus généralement toutes les perturbations respectant la symétrie des lignes de courant n'auront que peu d'effet sur cellesci et n'abaisseront en principe que peu la surtension théorique. Le champ $\vec{H}$ est dans ces modes dirigé préférentiellement suivant l'axe de la cavité, ce qui est favorable à un bon coefficient de remplissage. Les dimensions presque prohibitives d'une cavité résonnañt à $3035,7 \mathrm{MHz}$ sur les modes supérieurs, de surtension plus élevée, nous ont conduits à fixer notre choix sur le mode TE 011. La carte du champ correspondante est donnée dans l'appendice I. La cavité vide résonne alors pour un diamètre et une longueur égaux à 13,01 cm; la surtension théorique pour des parois de cuivre est $Q_{0} \simeq 54000$ et le coefficient de remplissage, calculé à l'appendice II, atteint la vaieuŕ maximium $n=0,856$ pour une cellule avec gaz étranger remplissant toute la cavité. Remarquons que la fréquence de résonance plus élevée de ${ }^{87} \mathrm{Rb}\left(\nu_{a}=6834,7 \mathrm{MHz}\right)$ a permis d'employer dans ce dernier cas une cavité résonnant sur le mode TE 021 tout en conservant des dimensions raisonnables $\left({ }^{4}\right)$. 
Nous avons également étudié le mode correspondant d'une cavité sphérique. qui résonne pour un diamètre de $14,1 \mathrm{~cm}$; la surtension théorique pour des parois de cuivre est $Q_{0} \simeq 58000$, et le coefficient de remplissage est égal à 0,798 dans les mêmes conditions que ci-dessus.

\section{2) Configurations étudiées}

Nous avons effectué nos premières expériences en plaçant une cellule de résonance d’un type classique au Laboratoire (sphères de $6 \mathrm{~cm}$ de diamètre), contenant le rubidium et le gaz tampon, à l'intérieur d'une cavité accordable en laiton argenté. La surtension $Q_{0}$ de celle-ci. égale à 40000 en l'absence de cellule, tombe à 300 une fois la cellule mise en place $\left({ }^{48}\right)$; le montage convient bien pour l'étude de la résonance 0-0 par détection optique, mais aucune mesure d'amplification radioélectrique n'est possible dans ces conditions. Cet effondrement de la surtension est lié aux pertes diélectriques et aux distorsions de la carte du champ $\vec{H}$ causées par les parois de la cellule. Nous nous sommes ainsi vite aperçus qu'il fallait considérer cellule et cavité comme un ensemble et les mettre au point simultanément.

La réalisatiori d'un tel ensemble s'est révélée très difficile et a constitué la partie la plus longue et la plus ingrate de notre travail. Les caractéristiques que doivent présenter la cellule et la cavité sont en effet les suivantes :

a) Cellule: - Etuvabilité et étanchóité rigoureuse après scellement, de manière à pouvoir contenir la vapeur de rubidium et le gaz tampon. La pression de vapeur du rubidium est en effet. d'environ $10^{-6}$ torr et ne pourrait subsister en présence d'une pression équivalente de gaz tels que l'oxygène ou la vapeur d'eau sur lesquels le rubidium réagit immédiatement.

- Possibilité d’un paraffinage des parois sous vide, destiné à minimiser la relaxation et la vitesse de disparition du rubidium sur celles-ci. 
- Réalisation en un matériau de pertes diélectriques et d'épaisseur les plus faibles possible pour ne pas affecter la surtension, amagnétique pour ne pas perturber l'homogénéité du champ directeur $\vec{H}_{0}$, et transparent à la lumière de pompage sur au moins une partie de la surface.

b) Cavité : - Résonance à $3035,7 \mathrm{MHz}$, avec une surtension élevée une fois la cellule mise en place, présence d'au moins une boucle de cauplage et possibilité d'accord.

- Présence de fenêtres transparentes, suffisamment dimensionnées, destinées au passage de la liumière de pompage.

- Réalisation en matériaux amagnétiques.

Les premiers essais montrèrent la difficulté de concevoir et de faire réaliser par l'industrie une cellule ne perturbant que peu la surtension de la cavité. L'idée de considérer cellule et cavité comme un seul ensemble fut alors portée à son point extrême en essayant de supprimer la cellule et d'introduire le rubidium directement dans une cavité étanche. Si le problème posé par l'effet des parois de la cellule sur la surtension se trouve ainsi résolu, les exigences d'étuvabilité, d'étanchéité et de paraffinage se trouvent alors reportées sur la cavité elle-même. Nous avons néanmoins tenté de mettre au point un tel ensemble. Le but initial de notre travail était en effet d'obtenir l'oscillation et nous ne pouvions repousser une solution qui conduit à la meilleure surtension.

Nous avons effectué de nombreux essais, dont les détails techniques ont été rassemblés dans l'appendice III. A la suite des résultats obtenus après chacun d'eux, nous avons au total entrepris l'étude des six configurations suivantes, schématisées sur la figure 12 (le système d'accord, non représenté, agit par déformation du fond souple fermant la cavité du côté droit, sauf dans la dernière configuration où il dé- 
place l'hémisphère droit).

Configuration A. Cavité cylindrique étanche en cuivre (diamètre intérieur $132 \mathrm{~mm}$, longueur intérieure $121 \mathrm{~mm}$ ) paraffinée intérieurement, contenant le rubidium et le gaz tampon (azote). Les fenêtres sont réalisées par soudure directe verre-cuivre, le minimum de Kovar (ferromagnétique) est employé pour les queusots et la boucle, tandis que l'étanchéité entre les fonds et le corps de la cavité est assurée par un joint d'or. L'avantage de cette solution est une excellente surtension $\left\{Q_{0} \simeq 45000\right\}$ et un bon coefficient de remplissage en présence de gaz étranger. L'étanchéité au niveau des joints d'or n'est malheureusement pas toujours sûre et la réalisation des soudures verre-cuivre a donné lieu à de nombreux déboires. Nous avons tenté d'observer l'oscillation sur deux cavités de ce type, avec un résultat négatif. A l'époque de cette expérience (Août 1965), nous n'avions pas encore mis au point la technique de mesure de l'amplification. mais l'étude par voie optique de la relaxation de $\langle\vec{S}$. $\vec{I}>$ et de la résonance 0-0 nous a montré que la tension de vapeur de rubidium dans les deux cavités était très insuffisante, à la suite d'une rentrée d'air par plusieurs fuites. Une solution voisine est actuellement étudiée dans un autre laboratoire pour un maser à ${ }^{87} \mathrm{Rb}\left(4^{49}\right)$.

Configuration B. Cavité cylindrique étanche en acier inoxydable amagnétique cuivrée et paraffinée intérieurement; fenêtres réalisées par soudure directe verre-acier inoxydable. fermeture par joints d'or. Par rapport au modèle précédent, cette configuration doit donner une étanchéité plus sûre au niveau du joint d'or; la réalisation des soudures verre-inox est par contre encore plus difficile que celle des soudures verre-cuivre. Nous n'avons pas encore pu faire les essais correspondants. C'est une solution de ce type (sans paraffinage) qui a conduit à la première réalisation d'un maser autooscillateur à ${ }^{87} R b\left({ }^{3}\right)\left({ }^{4}\right)$. Notons que, dans cette dernière 


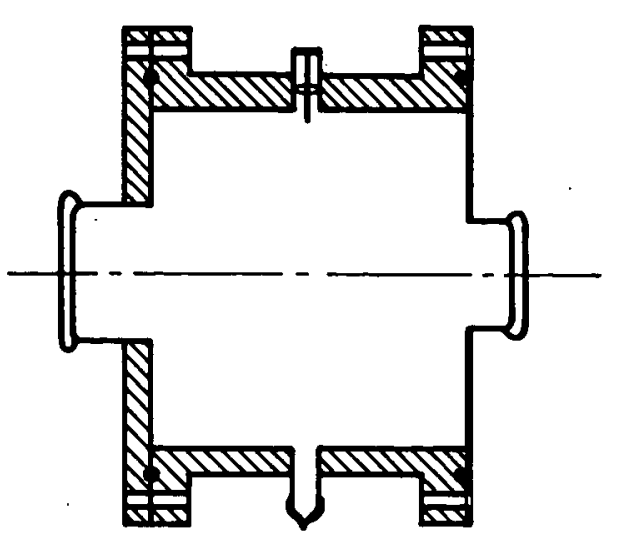

A

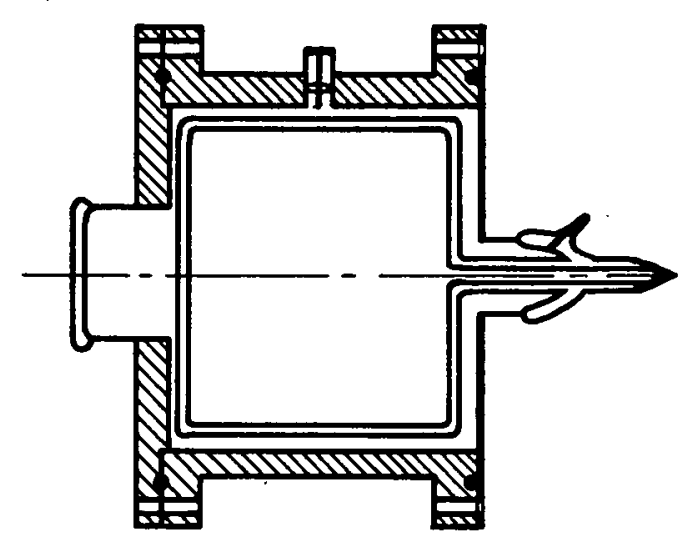

C

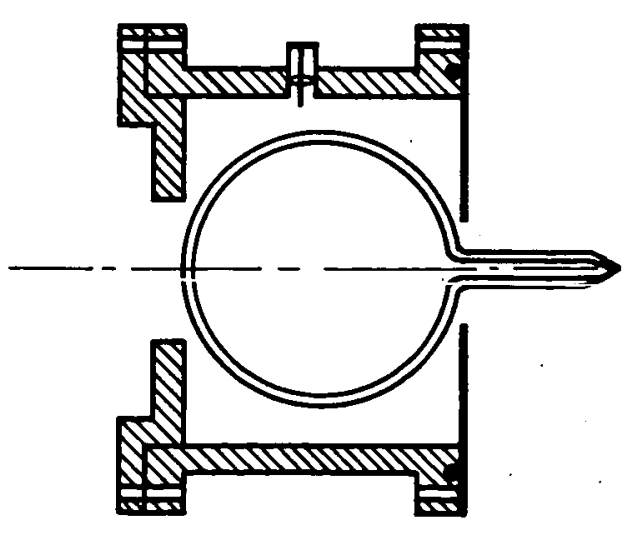

$E$
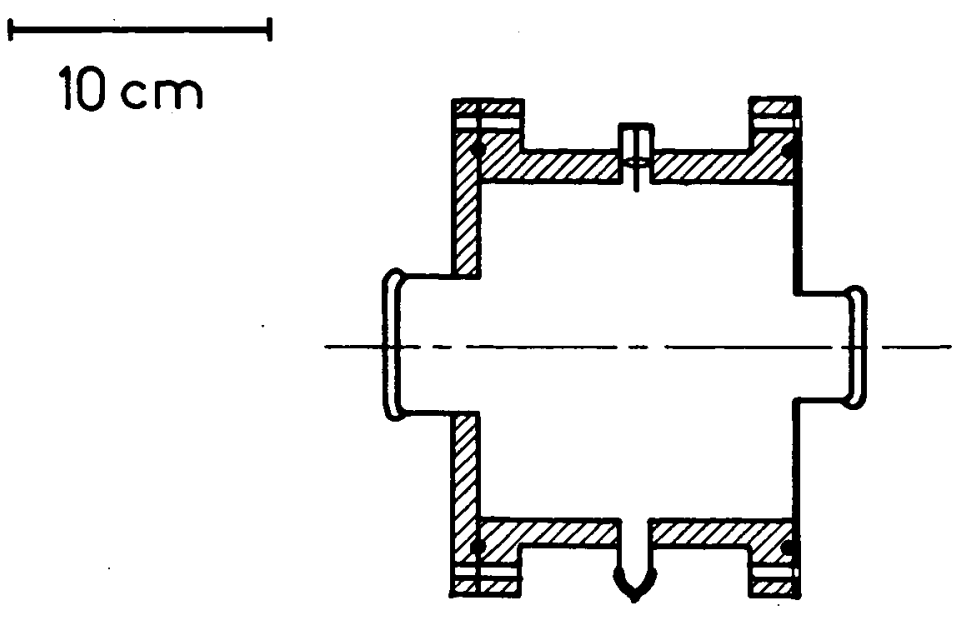

B

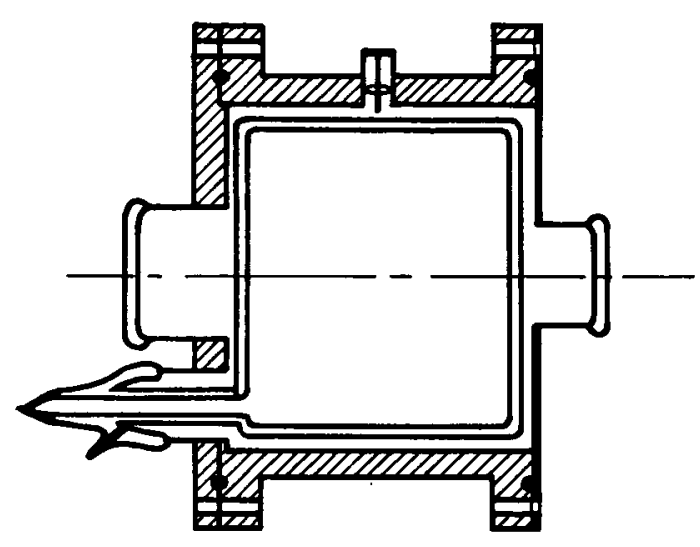

D

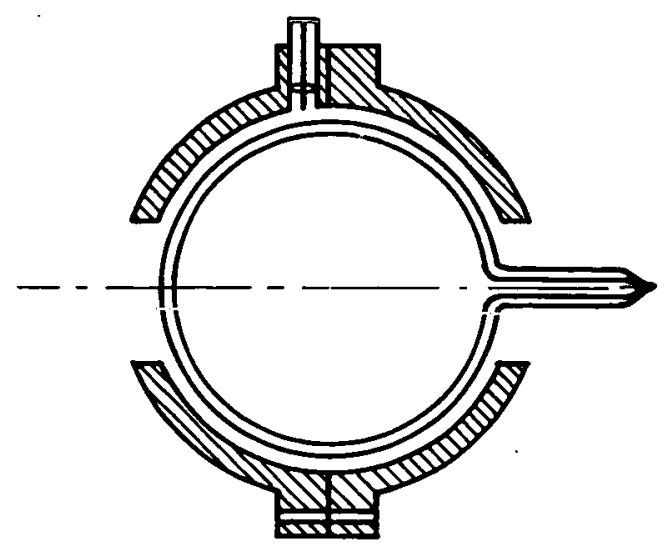

F

Fig.12 
expérience, la quantité importante $(50 \mathrm{mg})$ de ${ }^{87} \mathrm{Rb}$ introduite lors du remplissage $n^{\prime a}$ permis le fonctionnement que pendant environ un mois; il existait donc une cause importante de disparition du rubidium (fuites, ou absorption par les parois) qui a empêché jusqu'à présent une étude détaillée des propriétés de l'oscillation. Configuration C. Cavité cylindrique étanche en cuivre, renfermant une cellule en silice fondue transparente paraffinée intérieurement, contenant elie-même le rubidium et le gaz tampon. La cellule, cylindrique, a des parois très minces et très proches de la surface intérieure de la cavité. Celle-ci, de même structure que dans la configuration A, maintient un prévide autour de la cellule et la protège ainsi contre l'écrasement par la pression atmosphérique. La cavité doit encore résister à l'étuvage, puisque le prévide doit être établi dès que l’intérieur de la cellule est mis sous vide; mais le gros avantage est que, par suite de l'étanchéité de la cellule, les fuites très faibles de la cavité ont beaucoup moins d'importance que dans le cas précédent. Par contre, la surtension est un peu abaissée $\left(Q_{0} \simeq 35\right.$ 000), la réalisation des cellules pose des problèmes difficiles et l'assemblage final de la double enceinte à vide est acrobatique. Certaines des mesures d'amplification que nous avons effectuées l'ont été avec un ensemble de cette configuration qui, après optimisation de tous les facteurs, a permis actuellement d'atteindre un paramètre d'amplification $p=0,86$. Configuration D. Cavité cylindrique étanche en acier inoxydable cuivré, renfermant uñe dellule cominie précédemment muis comportant deux fenôtres pour le pompage nptirille. Cette dernière particularité doit, en permettant une meilleure illumination, améliorer beaucoup l'efficacité du pompage $\alpha$ et le coefficient de remplissage effectif $n$ par rapport à la configuration précédente. Nous n'avons pas encore pu en faire l'essai. 
Configuration E. Cavité cylindrique en cuivre ouverte à l'atmosphère, renfermant une cellule sphérique en silice à parois minces. La forme sphérique permet à la cellule de résister à l'écrasement par la pression atmosphérique sans qu'il soit nécessaire d’établir un prévide. Ceci constitue une énorme simplification et conduit à une souplesse d'emploi très supérieure : on peut, par exemple, ajouter une seconde boucle de couplage non prévue au départ, connecter les câbles sans craindre de briser la fragile perle de verre d'un couplage étanche, etc. Par contre, les parois de la cellule traversent cette fois des zones de la cavité où le champ électrique hyperfréquence est notable et non plus nul comme au voisinage des parois; il en résulte une surtension nettement mains élevée $\left(Q_{0} \simeq 18000\right)$ et par ailleurs la diminution du rapport $V_{\text {cell }} N_{\text {cav }}$ risque de retentir sur le coefficient de remplissage $n$. Nous avons néanmoins tenu à entreprendre l'étude de cette configuration, qui a été la première fen Janvier 1966) dont nous avons pu obtenir une réalisation satisfaisante. Elle a été utilisée dans de nombreuses expériences et a conduit jusqu’à présent à un paramètre d'amplification optimum $p=0,55$.

Configuration F. Cavité sphérique ouverte en cuivre, renfermant une cellule sphérique en silice fondue comme dans la configuration précédente. Les parois de la cellule se trouvant de nouveau au voisinage de celles de la cavité, la surtension est nettement améliorée $\left(Q_{0} \simeq 40\right.$ 000) et le coefficient de remplissage peut-être également. Le calcul et la réalisation d'une cavité sphérique sont par contre peu classiqueset la construction du système d'accord est plus délicate. Plusieurs expériences ont été effectuées avec cette configuration, pour laquelle nous avons actuellement mesuré à’ l'optimum un paramètre d'amplification $p=0,88$. 


\section{3) Technique de remplissage}

Une fois construit un ensemble de configuration donnée, il reste à effectuer le remplissage de la cellule ou de la cavité étanche. La technique s'inspire de celle qui a été mise au point dans le cas de cellules sphériques en Pyrex de petit diamètre $\left({ }^{5}\right)\left({ }^{6}\right)$. L'étuvage à $400^{\circ} \mathrm{C}$ de cellules sphériques en silice ne pose pas de problèmes. Par contre, dans le cas de cavités étanches, les scellements verre-métal supporteraient mal des températures supérieures à $350^{\circ} \mathrm{C}$; l'étuvage d'une masse de métal dépassant $10 \mathrm{~kg}$, reliée au banc de pompage par des queusots de petit diamètre, dure alors couramment une quinzaine d'heures. Le paraffinage s'opère en chauffant par induction sous vide une tige métallique portant la paraffine sur son extrémité placée au centre de la cellule. La grande surface à recouvrir rend l'opération assez longue, mais des essais sur une cellule verre-cuivre de petit diamètre ont montré que la paraffine semblait se déposer également bien sur le cuivre et former un revêtement efficace. Le rubidium 85 , enrichi à $99,54 \%$, est préparé par la réaction sous vide habituelle RbCl $+\mathrm{BaN}_{6}$, puis distillé dans le queusot : quelques milligrammes suffisent. On sait qu'une paroi paraffinée ne voit ses propriétés se stabiliser qu'après une "période de formation" au contact de la vapeur de rubidium ( ${ }^{50}$ ). Celle-ci durant un temps considérable en présence de gaz étranger (deux mois pour notre première cellule contenant 3,4 torrs d'azotej, nous avons été amenés à prévoir, sur le queusot de toutes nos cellules, un joint que l'on peut briser sous vide, de type "queue de cochon", permettant de:n' introduire le gaz étranger qu'une fois la "formation" terminée; en l'absence de gaz, la durée de celle-ci se trouve ramenée à quelques jours. 
Dans les chapitres précédents, nous avons décrit le système atomique qui nous intéresse à l'aide d'un modèle dont la simplicité nous a permis de pousser les calculs assez loin et avons étudié la constitution du montage expérimental permettant de vérifier les conclusions obtenues. Nous nous proposons maintenant d'exposer les résultats des mesures et de les interpréter.

\section{A) GENERALITES}

Nous nous rappelons que tous les calculs précédents ont été effectués moyennant une hypothèse fondamentalefaite au chapitre I : le système atomique peut être représenté par une matrice densité moyenne $\sigma$. Il est temps maintenant d'examiner quelle en est la validité et pour cela de préciser quel est le mouvement des atomes alcalins dans la cellule. En l'absence de gaz étranger, les atomes vont en ligne droite d'une paroi à l'autre en un temps qui, à la vitesse moyenne $\bar{v}=3.10^{4} \mathrm{~cm} / \mathrm{s}$, est de quelques $10^{-4} \mathrm{~s}$; de plus, la probabilité de relaxation lors d'un choc sur la paroi recuuverte de paraffine est très faible. Il en résulte qu'après un temps de $I^{\prime}$ ordre de $10^{-3}$ seconde, l'atome aura "moyenné" les conditions physiques existant aux différents points de la cellule et en particulier les conditions d'illumination, puisque, comme nous le verrons, le temps de pompage $T_{p}$ (temps séparant l'absorption successive de deux photons par le même atome) n'est guère inférieur à $10^{-2} \mathrm{~s}$. Même si celles-ci ne sont pas uniformes, l'hypothèse d'une matrice densité moyenne $\sigma$ évoluant sous l'action d'interactions moyennes est alors parfaitement valable. Nous verrons à l'appendice II que cette 
conclusion s'étend à l'action du champ de radiofréquence $\vec{H}$ : on a alors $H_{1 z}=\left\langle H_{z}{ }^{>}\right.$cell , et $n=\left\langle H_{z}>_{\text {cell }}^{2} V_{\text {cell }} /<H^{2}\right\rangle_{\text {cav }} V_{\text {cav }}$.

La situation est très différente lorsque la cellule contient quelques torrs de gaz étranger. Le mouvement des atomes alcalins en ligne droite d’une paroi à l'autre est alors remplacé par.une lente diffusion au sein du gaz. Soit $n(\vec{r}, t)$ la densité d'atomes alcalins au point $\vec{r}$ à l'instant $t: n(\vec{r}, t)$ obéit à l'équation de diffusion :

$$
\frac{d}{d t} n(\vec{r}, t)=D \nabla^{2} n(\vec{r}, t)
$$

où $D$, coefficient de diffusion de l'alcalin dans le gaz, est relié au libre parcours moyen de transport $\ell_{t}$ par 1 'équation $D=\ell_{t} \bar{v} / 3\left({ }^{9}\right)$. La résolution de 1 'équation ci-dessus montre qu'un atome se trouvant au point $\vec{r}_{0}$ à l'instant $t_{0}$ se trouvera à l'instant $t$, en moyenne, sur une sphère centrée en $\vec{r}_{0}$ et de rayon $\left|\vec{r}-\vec{r}_{0}\right|$ donné par la relation $\left|\vec{r}-\vec{r}_{0}\right|^{2}=$ $40\left(t-t_{0}\right)$. Ainsi, dans une cellule à $40^{\circ} \mathrm{C}$ contenant 8 torrs d'azote, un atome de rubidium met $10^{-2}$ s pour s'éloigner d'un centimètre. Les temps de pompage $T_{p}$ ou de relaxation $T_{2}$ dans les conditions habitueljes sont précisément de cet ordre; il en résulte que l'atome ainsi confiné ne fait plus la moyenne des conditions physiques existant dans la cellule et que la mesure d'une observable déterminée sera relative non à l'ensemble des atomes, mais à une partie seulement de ceux-ci. Supposons par exemple que la cellule soit éclairée par un faisceau parallèle se propageant suivant l'axe de la cavité et de diamètre très inférieur à celui de la cellule, la densité des atomes alcalins étant faible : l'étude de l'absorption optique en l'absence de radiofréquente ne pūtera quie sur las atomes se trouvant dans le faisceau et non sur ceux qui restent dans l'obscurité au voisinage de la paroi latérale. Les mesures radioélectriques fourniront pour le paramètre d'amplification $p$ une valeur moyenne prise de façon différente; il est clair en particulier que le coefficient de remplissage $n$ (et donc p) n'aura une valeur notable 
que si les atomes en état d'inversion de populations se trouvent dans une région où la composante $\mathrm{H}_{z}$ du champ de radiofréquence est importante, ce qui est d’ailleurs le cas dans l'exemple choisi. Même en fait si les conditions physiques sont uniformes, les coefficients de remplissage en l'absence et en présence de gaz seront différents; dans ce dernier cas, nous verrons à l'appendice II que I'on a cette fois $\left.n=\left\langle H_{z}^{2}\right\rangle_{\text {cell }} V_{\text {cell }} /<H^{2}\right\rangle_{\text {cav }} V_{\text {cav }}$.

Le confinement des atomes compliquera ainsi l'interprétation des résultats fournis par les différentes méthodes d'observation du système atomique passées en revue aux chapitres précédenta, mais particulièrement celle des mesures d'amplification, dont nous avions déjà signalé le caractère incertain au chapitre III. La valeur moyenne de $p=K \alpha n n Q_{0} / \delta \omega_{a}$ ne sera pas reliée de façon simple aux valeurs moyennes des différentes observables intervenant dans l'expression ci-dessus, observables qui interagissent d'ailleurs les unes sur les autres lorsqu'on fait varier les conditions expérimentales. Augmentons par exemple la température de la cellule : le principal effet de cette variation est d'augmenter la tension de vapeur de l'alcalin. c'est-à-dire la valeur de la densité $n$. L'importance de la relaxation d'échange s'en trouve augmentée, ainsi que l'absorption lumineuse; il en résulte une modification de la valeur moyenne de l'inversion de populations $\alpha$. Celle-ci devient différente d'un point à l'autre de la cellule si l'absorption est forte, ce qui retentit sur la valeur du coefficient de remplissage $\eta$. Enfin, la largeur de raie $\delta \omega_{a}$ devient proportionnelle à la densité $n$ si celleci est assez grande pour que la relaxation d'échange domine : $n / \delta \omega_{a}$ tend donc vers une valeur limite.

Nous résumons ci-dessous les diverses interdépendances que nous rencontrerons lors de l'étude de l'amplification. les variations les moins importantes étant entre parenthèses (rappelons que les mesures d'amplification nous fournissent la valeur 
moyenne de $1 / Q_{a}=-K \alpha n n /\left(\delta \omega_{a}+i\left(\omega-\omega_{a}\right)\right)$, ou encore de $\left.p=K \alpha n \eta Q_{0} / \delta \omega_{a}\right)$.

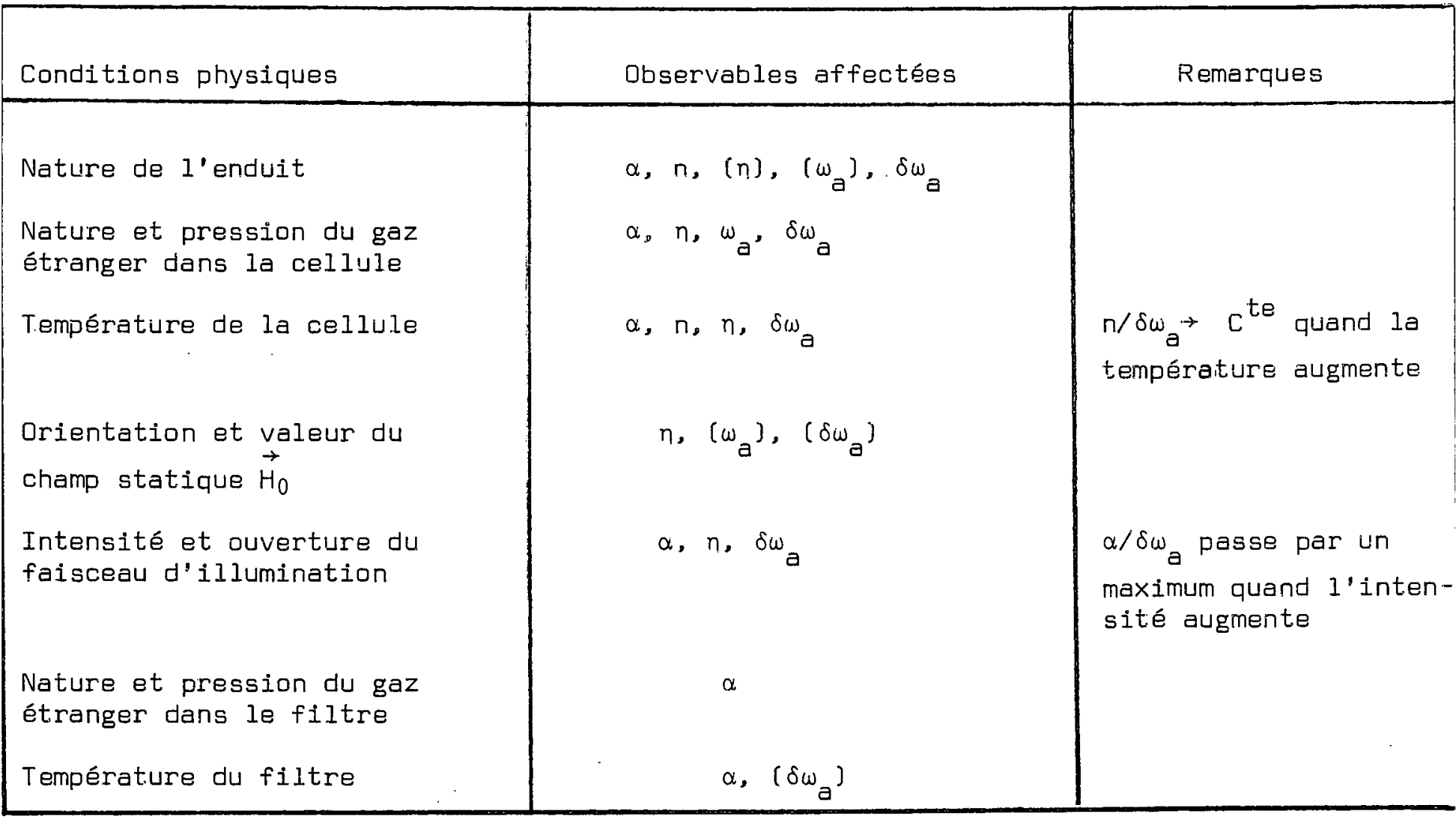

B) ETUDE DE L'ABSORPTION LUMINEUSE EN L'ABSENCE DE RADIOFREQUENCE

Cette étude s'est révélée extrêmement précieuse pour connaitre le comportement du système. Comme naus l'avons vu au chapitre II, elle permet en effet de déterminer le flux lumineux effectif $1 / T_{p}$ présent, dans la cellule, les temps de relaxation $T_{H}$ et $T_{\text {eH}}$ ' la densité $n$. la qualité $x$ de la lumière de pompage et l'inversion de populations limite $\alpha_{m}$. La méthode est utilisée systómatiquement au laboratoire depuis de nombreuses années, ce qui nous a permis des comparaisons instructives. Il faut, pour l'appliquer, mesurer simultanément : 
a) Les caractéristiques de l’exponentielle de pompage : évolution dë la lumière transmise par la vapeur lorsqu'on ouvre brusquement l'obturateur, fermé au préalable pendant un temps assez long. On mesure sur la photographie l'amplitude initiale de l'exponentielle, proportionnelle à $\tilde{L}_{A}-\hat{L}_{A}$ et la constante de temps $\tau_{p}$ ( $f i g$. 13). b) La lumière absorbée $\hat{L}_{A}$. On mesure pour cela la transmission lumineuse dans les conditions de l'expérience, puis après avoir refroidi la cellule en-dessous de $10^{\circ} \mathrm{C}$ de manière à annuler la tension de vapeur de rubidium : l'absorption s'obtient par différence. Cette méthode a l'inconvénient de nécessiter que l'intensité et le régime de la lampe ne varient pas pendant le temps assez long (plusieurs heures dans le cas d'une cavité métallique) nécessaire au refroidissement; elle est cependant la seule praticable dans notre cas, où nous ne disposions pas de cellules identiques mais vides que l'on pourrait substituer à la cellule étudiée.

Nous avons effectué cette étude pour toutes nos cellules et en présentons ci-après les conclusions générales en les illustrant par des exemples caractéris-. tiques.

1) Variation de la constante de temps de pompage T $\mathrm{q}$ avec l'intensité d'iliumination

La figure 14 représente la variation de l'inverse de la constante. de temps $\tau_{p}$ avec l'intensité d'illumination. La mesure a été effectuée sur une cellule sans gaz étranger (courbe b) et sur la même cellule à la même température $\left(27^{\circ} \mathrm{C}\right.$ ) contenant 6,3 torrs d'azote, introduits comme il a été expliqué au chapitre précédent; dans ce dernier cas, le faisceau de pompage était soit parallèle lors de la traversée de la cellule (courbe a), soit fortement divergent à partir d'un point situé sur la face d'entrée (courbe $a^{\prime}$ ). Conformément à la formule $1 / \tau_{p}=1 / T_{1 H}+1 / T_{p}$, démontrée au chapitre II pour une cellule avec gaz étranger, cette variation est linéaire dans 


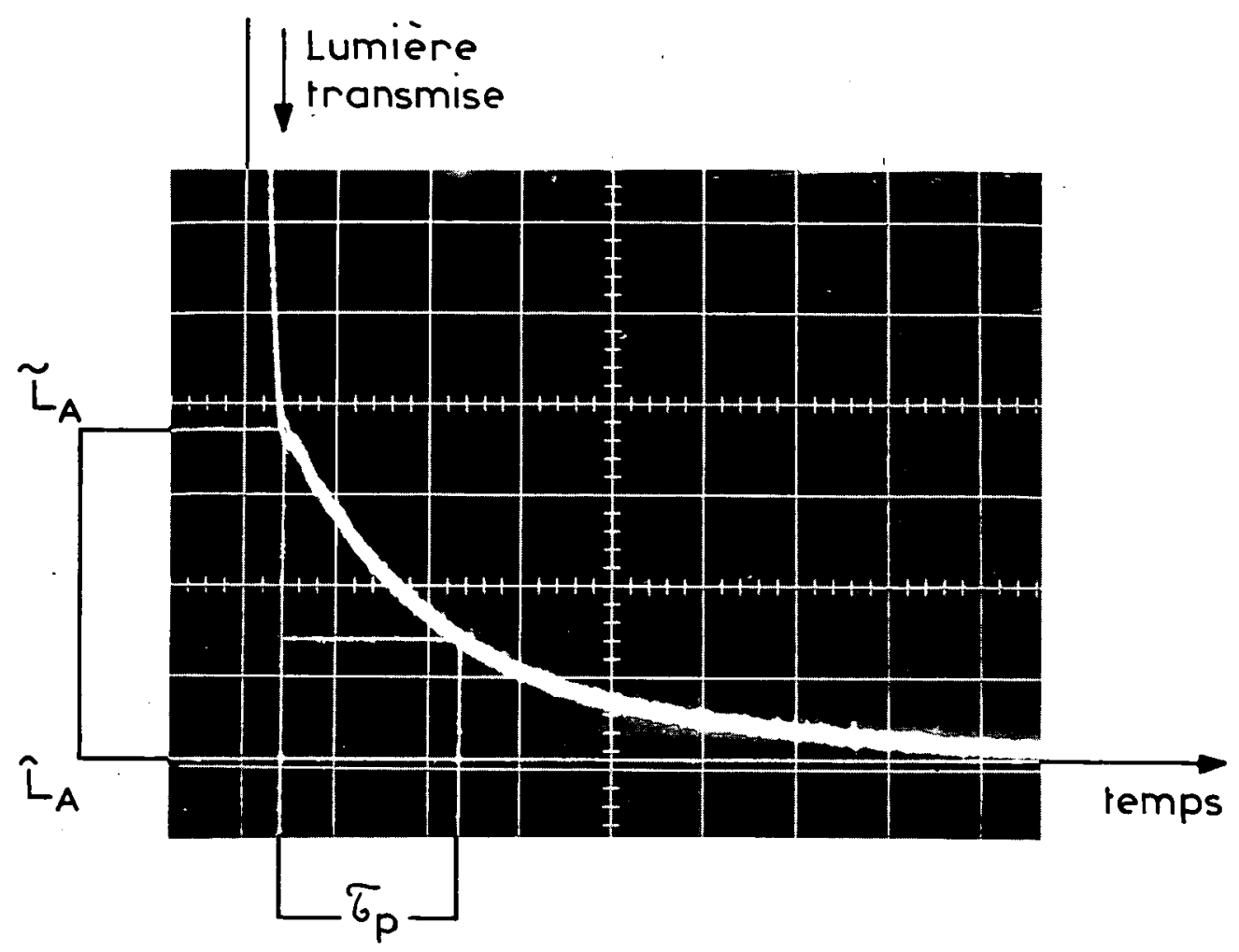

Fig. 13 


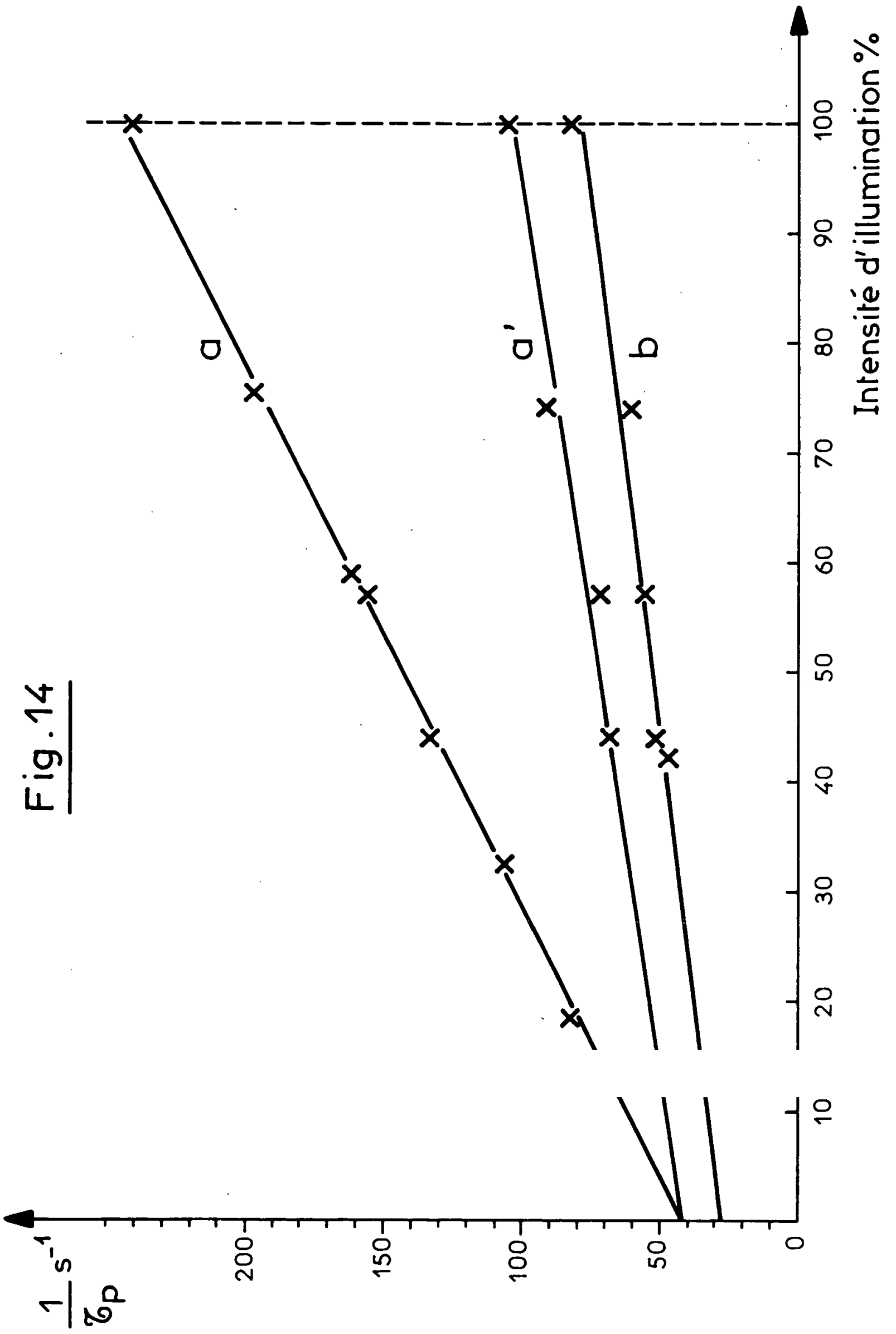


les cas a et $a^{\prime}$. Le calcul ne s'applique pas aux cellules sans gaz étranger, mais l'expérience montre que l'évolution au cours du temps de la lumière absorbée par ces cellules est encore sensiblement exponentielle. La courbe b montre que la variation de la constante de temps $\tau_{p}$ avec l'intensité d'illumination est également linéaire, ce qui nous permet de définir ici aussi un temps de pompage $T_{p}$ inversement proportionnel à l'intensité d'illumination. Quelle que soit d'ailleurs la loi suivie, l'extrapolation à une intensité lumineuse nulle fournit la valeur $1 / T_{1 H}$ de l'inverse du temps de relaxation de $\langle\vec{S} . \vec{I}>$. La figure suggère les remarques suivantes.

a) En présence de gaz et à intensité lumineuse donnée, le temps de pompage $T_{p}$ est beaucoup plus court en géométrie d'illumination parallèle (droite a) qu'en géométrie divergente (droite $a^{\circ}$ ). Ceci met en évidence de façon frappante l'emprisonnement dû au gaz. Nous avons vu en effet qu'un atome alcalin diffusant au sein du gaz met un temps très long pour parcourir quelques centimètres. Avec la géométrie divergente que nous utilisions, le faisceau s'étalait très vite et éclairait tout l'intérieur de la cellule à peu près uniformément, tant du moins que l'absorption optique était faible. Le temps de pompage $T_{p}$ est alors assez long, approximativement égal d'ailleurs à celui que nous avons pu définir dans le cas dPune cellule sans gaz. Par contre, en géométrie parallèle, le faisceau utilisé avait un diamètre d'environ $5 \mathrm{~cm}$. Les atomes situés au voisinage de l'axe étaient donc éclairés en permanence par la totalité du flux, sans avoir le temps de diffuser, tandis que les atomes situés près des parois restaient dans l'ombre. La detection optique n'ubservant que les premiers, 11 leur correspondait un temps de pompage $T_{p}$ beaucoup plus court, On prévoit ainsi une grande influence de la présence du gaz et de la géométrie d'illumination sur l'inversion de populations $\alpha$, le coefficient de remplissage $n$, et donc sur la valeur du paramètre d'amplification p moyennée sur. l'ensemble de la cellule. 
b) Le temps de relaxation $T_{1 H}$ dû aux collisions sur les parois et aux collisions d'échange dans la cellule sans gaz étranger est égal à $3,7.10^{-2} \mathrm{~s}$; en présence de gaz, il se raccourcit et devient égal à $2,38 \cdot 10^{-2} \mathrm{~s}$. On sait ( ${ }^{9}$ ) que la présence des molécules du gaz étranger peut avair deux effets contraires : elle diminue l'importance de la relaxation due aux parois en ralentissant le mouvement des atomes vers cellesci, mais peut être elle-même une cause nouvelle de relaxation lors des collisions contre les atomes de l'alcalin. Le second effet l'emporte ici sur le premier, contrairement à ce qui se produit pour les cellules à parois non paraffinées dont la relaxation très énergique est réduite par l'emploi d’un gaz tampon.

2) Variation du temps de pompage $T$ avec la température de la cellule La variation, en fonction de la température, de l'inverse $1 / T_{p m} d u$ temps de pompage correspondant à l'illumination maximum est représentée sur la figure 15, pour la même cellule contenant 6,3 torrs d’azote. Les températures ont été portées en abscisses suivant une échelle proportionnelle à la tension de vapeur saturante du rubidium qui leur correspond $\left(5^{1}\right)$, représentation qui $5^{\circ e s t}$ révélée physiquement très parlante. Les lettres a et a' se réfèrent aux mêmes cas que dans la figure précédente. Aux basses température de la cellule, $1 / T_{p m}$ est très différent en géométrie parallèle (courbe a) ou dịvergente (courbe a'), comme nous $1^{\prime}$ avons vu sur les courbes de la figure précédente prises à $27^{\circ} \mathrm{C}$. Aux températures élevées au contraire, $1 / T$ pm devient insensible à la géométrie d'illumination. Ceci sexplique si l'on se souvient que l'absorption lumineuse devient alors importante : les atomes soumis effectivement au pompage optique se trouvent confinés au voisinage de la face d'entrée et le volume observé ne dépend plus guère de la géométrie d’illumination. Le champ de radiofréquence $\vec{H}$ étant très faible dans cette région. on prévoit que le coefficient de remplissage $n$ et le paramètre d'amplification p seront en ce cas très petits. 


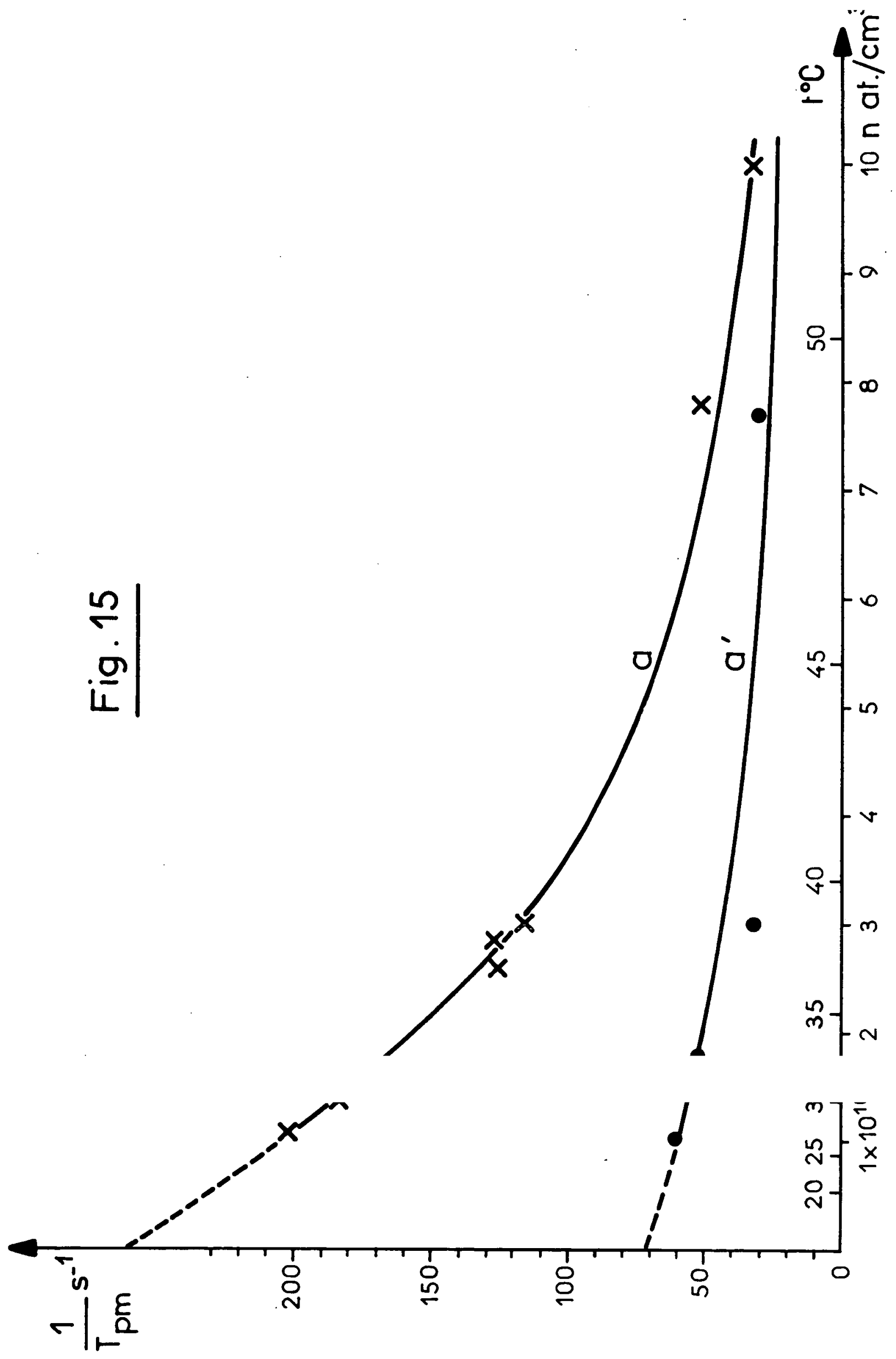




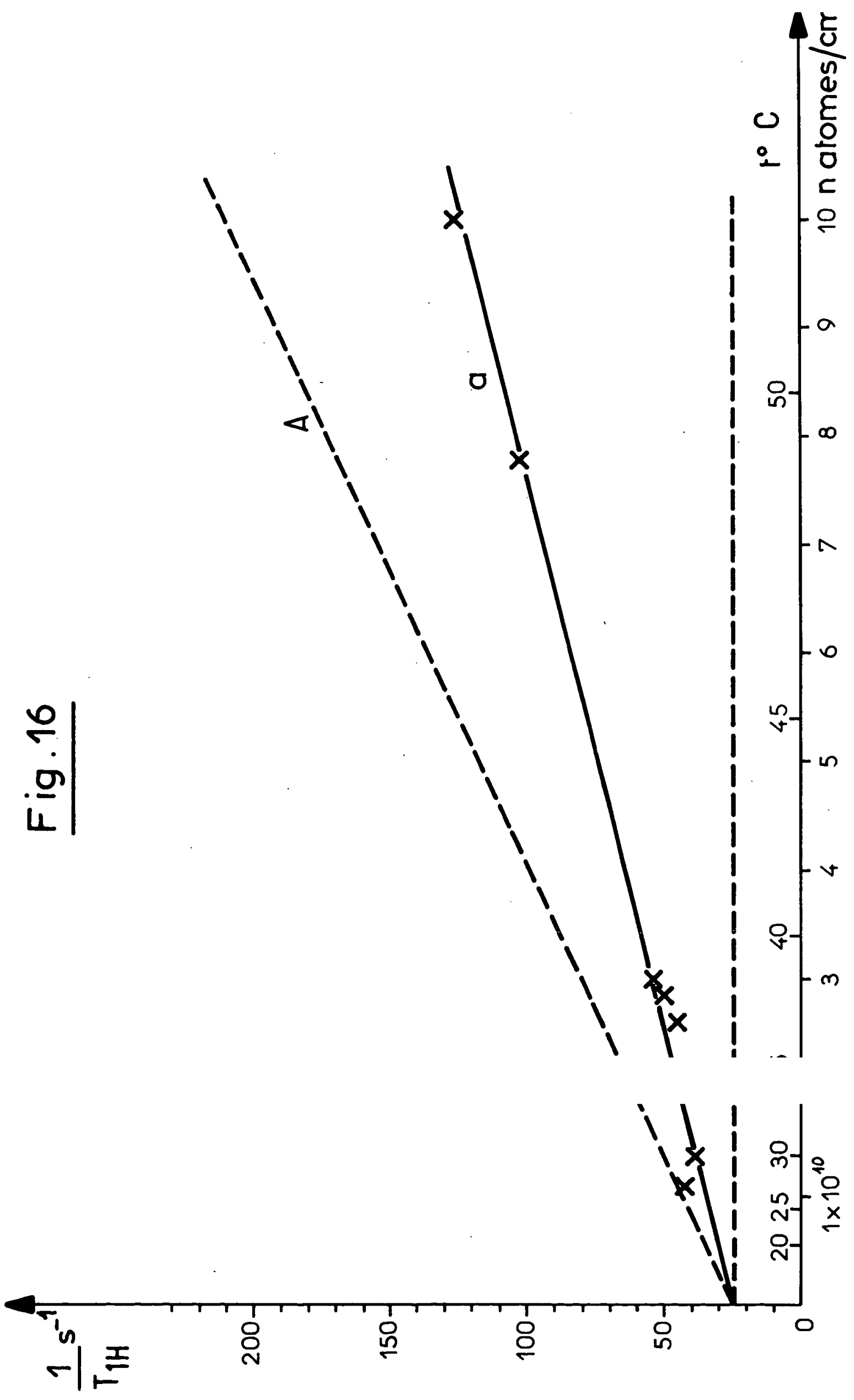


3) Variation du temps de relaxation $T_{1 H}$ avec la temperature de la ceilule

La figure 16 (courbe a) représente la variation de $1 / T_{1 H}$ en fonction de la température, pour la même cellule que précédemment. Nous avons vu au chapitre II que $1 / T_{1 H}=1 / T_{H}+1 / T_{e H}$, où $T_{H}$ décrit l'ensemble des processus de relaxation liés à la parai et au gaz, tandis que $T_{e H}$ rend compte des collisions d'échange; par ailleurs, ie temps de relaxation d’échange varie suivant la loi :

$$
\frac{1}{T_{\text {eH }}}=\bar{v} \pi \sigma_{\text {éch }}^{2} n
$$

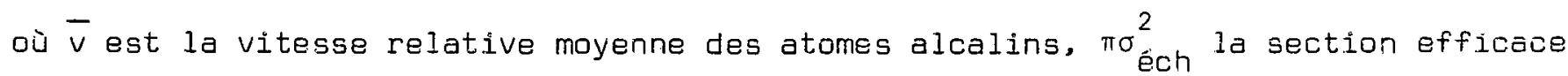
d'échange et $n$ la densité d'atomes alcalins. Dans une cellule remplie de vapeir saturante, $1 / T_{1 H}$ devrait alors varier linéairement avec $n$, suivant la droite $A$ de la figure 16 . Pour la cellule étudiée, nous trouvons expérimentalement que la variation est linéaire (droite a) mais de pente plus faible. Ceci nous montre qu'il existe dans la cellule un déficit de tension de vapeur par rapport à la tension de vapeur saturante; le rapport entre les deux est environ 0,53 dans toute la gamme de températures étudiée. Nous avons ainsi le moyen d'obtenir la valeur du paramètre très important $n$, intervenant en particulier dans la condition d'accrochage et nous avons appliqué le méthode à toutes nos cellules. Ceci nous a permis de constater que la valeur ci-dessus n'était atteinte ou dépassée que par des cellules dont la "formation", telle qu'elle a été décrito au chapitre précédent, avait été bien menée: sinon, la tension de vapeur est généralement très inférieire, et dépend de l'histoire antérieure de la cellule.

L'extrapolation de $1 / T_{1 H}$ pour une tension de vapeur nulle nous fournit la vaieur de ${ }^{T}{ }_{H}$, égal ici à $4 \cdot 10^{-2}$ s. Comme nous le verrons plus loin, nous n"avons pu obtenir une amplification radioélectrique notable qu'en présence d'une tension de vapeur élevée; I'effet de l'échange est alors prédominant dans la relaxation et TH est très inférieur à la valeur ci-dessus. 
4) Variation de l'amplitude de l'exponentielle de pompage $\hat{L}_{A}-\hat{L}_{A}$ avec I’intensité d’illumination

La variation du quotient de $\tilde{L}_{A}-\hat{L}_{A}$ par l'intensité d'illumination en fanction de celle-ci est représentée sur la figure 17. On voít qu"il devient constant aux fortes intensités ("saturation lumineuse"). Comme nous l'avons vu au chapitre II, l'effet. de la lumière est alors prépondérant dans la relaxation et tout accroissement de l'intensité diminue le temps de pompage $T_{p}$ sans aijgmenter appréciablement la différence de populations atteinte. Lorsque la densité n de la vapeur alcaline est élevée. l’absorption réduit le flux lumineux moyen présent dans la cellule et le temps de relaxation $T_{1 H}$ est court par suite de $1^{\circ}$ importance des collisions d'échange : la saturation devient dans ce cas difficile à réaliser.

5) Variation de l'amplitude de l'exponentielle de pompage $\hat{L}_{A}-\hat{L}_{A}$ et de la lumière absorbée $\hat{L}_{A}$ avec la température de la cellule

Cette variation est représentée sur la figure 18, toujours pour la même cellule contenant 6,3 torrs d'azote. Lorsque la température augmente, l'absorption augmente régulièrement, tandỉs que $l^{\circ}$ amplitude de l'exponentielle de pompage passe par un maximum : nulle pour $n=0$, lorsque l'absorption est elle-même nulle, la quantité $\tilde{L}_{A}-\hat{L}_{A}$ doit en effet s'annuler également lorsque $n$ est grand et que l.'absorption des composantes utiles devient totale quel que soit l'état interne du système atamique. Au-

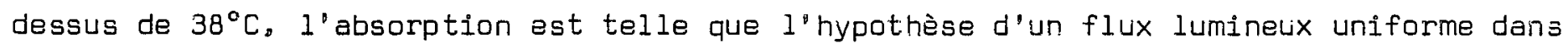
la cellule n'est plus valable et par ailleurs, la saturation lumineuse n'est plus réalisée dans les conditions de $l^{\circ e x p e ́ r i e n c e ~: ~ n o u s ~ a v o n s ~ t r a c e ́ ~ e n ~ p o i n t i l l e ́ s ~ l a ~ p a r t i e ~ c o r-~}$ respondante de la courbe. Au-dessous de cette température. on peut par contre appliquer Ies formules (12) du chapitre II. Nous voyons sur le figure 18 que $\left[\hat{L}_{A}-\hat{L}_{A}\right.$ ) / $\hat{L}_{A}=m$ est sensiblement constant et égal à 0,16 ; nous lisons alors sur la figure 3 que 1 'ef- 


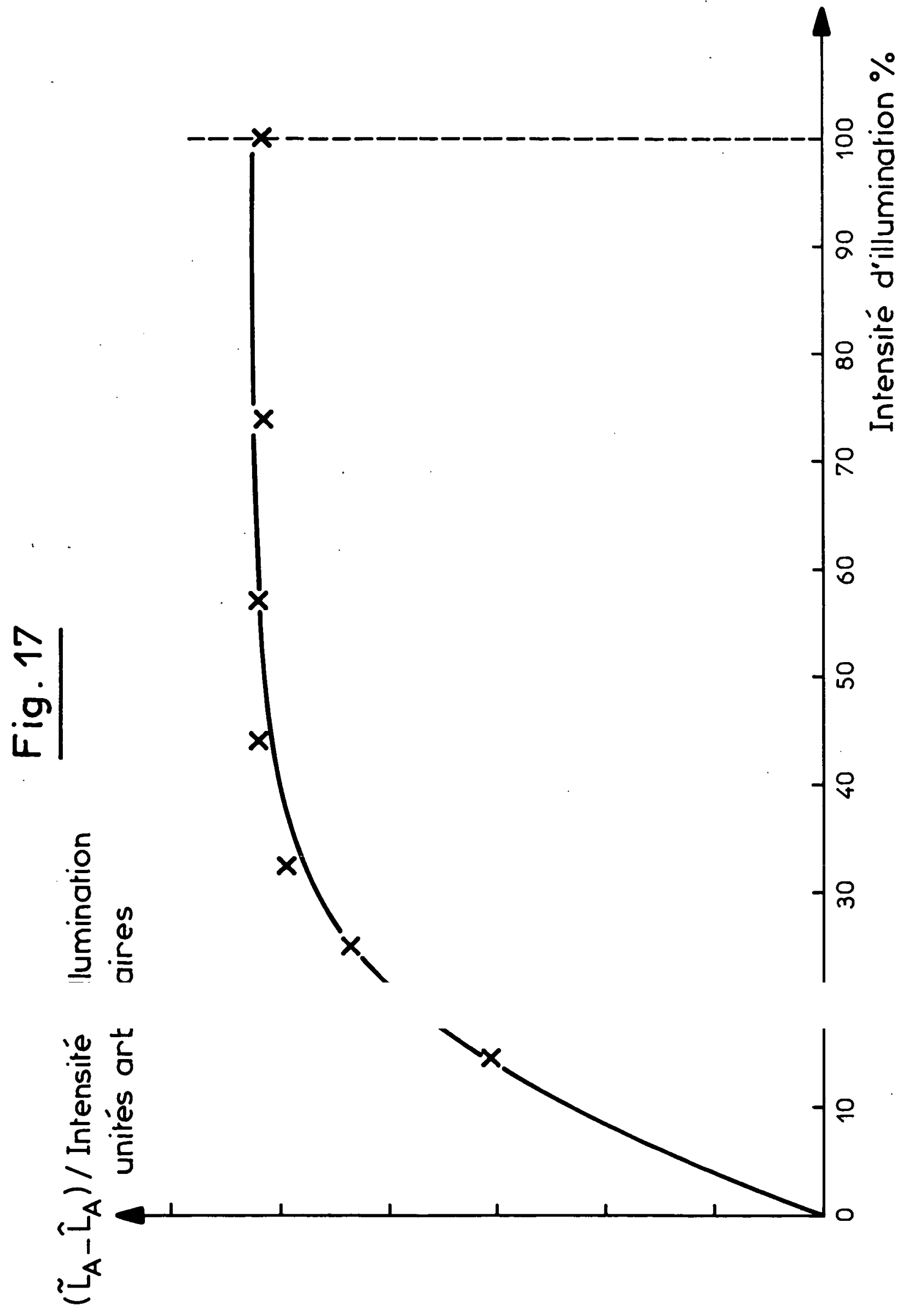




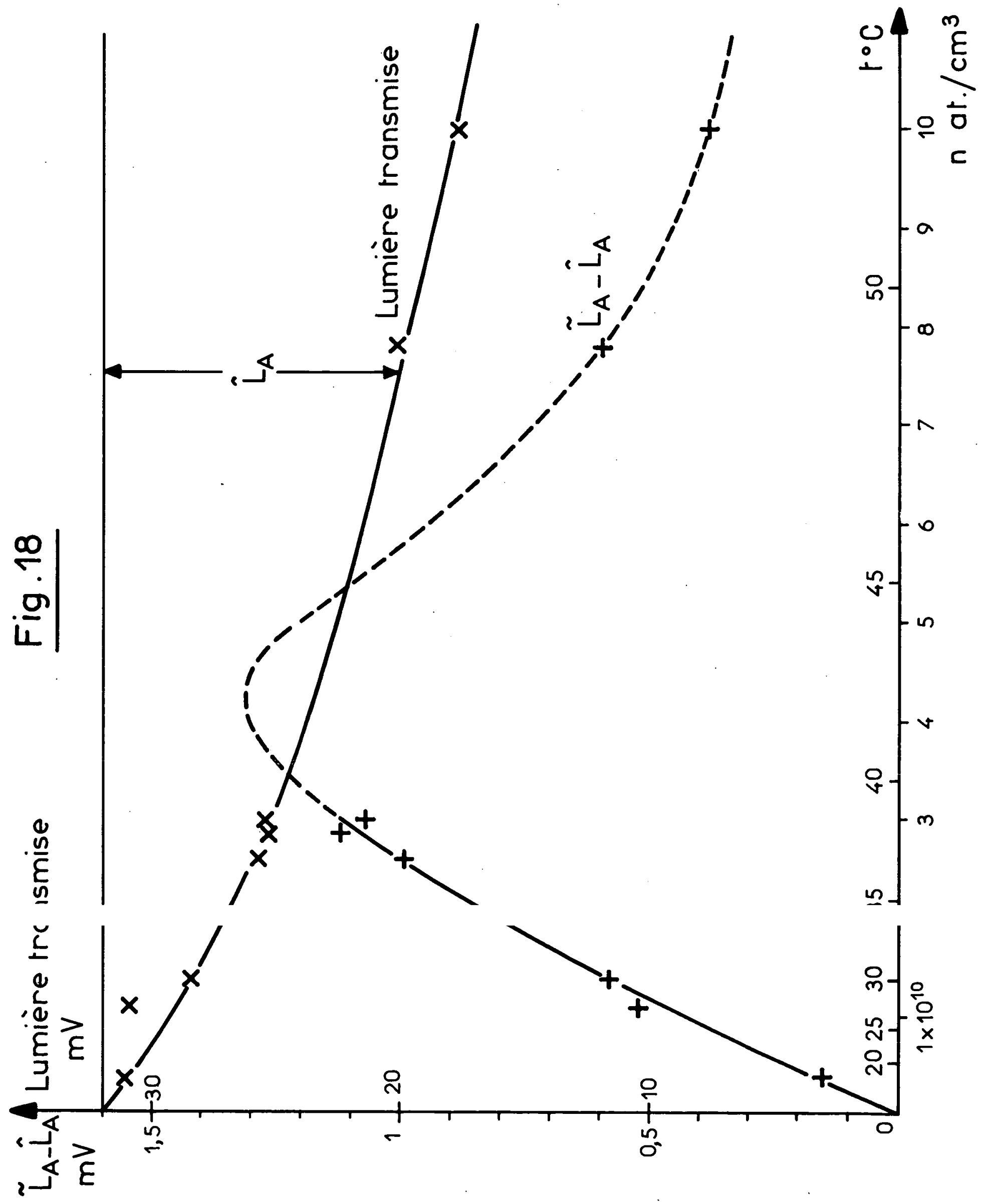


ficacité du filtrage hyperfin est $x=2,15$ et que l'inversion de populations limite $\alpha_{m}$ atteinte entre les sous-niveaux $\mid F_{+}, 0>$ et $\mid F_{-}, 0>$ est de $40 \%$ du maximum théorique égal ici $(I=5 / 2)$ à $1 / 7$, soit $\alpha_{m}=0,057$.

On peut se demander par comparaison quelles sont l'efficacité du filtrage et l'inversion atteintes lors du pompage hyperfin de ${ }^{87} \mathrm{Rb}$, dont le mécanisme est très semblable comme nous l'avons vu au chapitre précédent. Un calcul et des expériences se rapportant à une cellule à ${ }^{87} \mathrm{Rb}$ sans gaz étranger ( ${ }^{52}$ ) indiquent dans ce cas une valeur de $x$ égale à 3; par ailleurs, des mesures d'absorption en lumière blanche sur une cellule à ${ }^{87} \mathrm{Rb}$ contenant 11 torrs d'azote $\left({ }^{4}\right)$ conduisent, pour une température de $33^{\circ} \mathrm{C}$, à une inversion relative de populations $\alpha \simeq 0,125$. Toutes ces études optiques ne sont plus interprétables pour des températures de la cellule et donc des densités de vapeur élevées, nécessaires, comme nous le verrons, à l'obtention d'une forte amplification maser; la comparaison des conditions réalisées dans nos expériences d'amplification dont nous parlerons plus loin et de celles qui conduisent à l'autooscillation de ${ }^{87} \mathrm{Rb}$ semble toutefois confirmer que l'efficacité du filtrage $x$ et l'inversion de populations $\alpha$ obtenues sont plus grandes pour ${ }^{87} \mathrm{Rb}$ que pour ${ }^{85} \mathrm{Rb}$. Le meilleur filtrage réalisé dans le premier cas peut s'expliquer, comme nous l'avons vu au chapitre précédent. Il faut de plus remarquer que, d'après la formule (12-2), l'inversion de populations limite $\alpha_{m}$ à $x$ donné est également supérieure pour. ${ }^{87} \mathrm{Rb}(I=3 / 2)$; en particulier, la valeur maximum théorique est de $1 / 5$ pour ${ }^{87} \mathrm{Rb}$, contre $1 / 7$ pour ${ }^{85} \mathrm{Rb}$.

\section{C) DETECTION OPTIQUE DE LA RESONANCE D-D}

Ce procédé a été.très étudié dans de nombreux laboratoires en raison de son emploi dans les horloges atomiques à pompage optique. Nous l'avons surtout utilisé pour les réglages préliminaires : recherche et dégrossissage des conditions d'observa- 
tion de la raie, l'étude se poursuivant par détection radioélectrique. Signalons cependant deux expériences plus complètes.

1) Déplacement de la résonance 0-0 sous l'effet d'un gaz étranger

Nous avons, au chapitre I, écrit l'interaction hyperfine dans l'état fondamental $d^{P}$ un atome alcalin (un seul. électron s) sous la forme $W=A$. $\vec{S} . \vec{I}$. La constante A et la fréquence hyperfine $v_{a}$ sont données par :

$$
\left\{\begin{array}{l}
A=\frac{16 \pi}{3} \mu_{B}^{2} g_{I}|\psi(0)|^{2} \\
\nu_{a}=(I+1 / 2) A
\end{array}\right.
$$

$|\psi(0)|^{2}$ désignant la probabilité de présence de l'électron au niveau du noyau. Si l'atome subit des collisions contre les molécules d'un gaz étranger, la probabilité $|\psi(0)|^{2}$ s'en trouve légèrement modifiée et il en résulte un déplacement de la fréquence hyperfine $\nu_{a}(53)$. En appelant $p$ la pression du gaz étranger, on a :

$$
\frac{d \nu}{d p}=(I+1 / 2) \frac{d A}{d p}=\frac{16 \pi}{3} \mu_{B}^{2}(I+1 / 2) g_{I} \frac{d|\psi(0)|^{2}}{d p}
$$

Pour deux isotopes (1) et (2) d'un même élément et un gaz étranger donné, les quantités $|\psi(0)|^{2}$ et d $|\psi(0)|^{2} /$ dp sont les mêmes. On peut danc écrire dans ce cas :

$$
\frac{\frac{d v_{a}^{(1)}}{d p}}{\frac{d v_{a}^{\left({ }^{2}\right)}}{d p}}=\frac{\left(I^{(1)}+1 / 2\right) g_{I}^{(1)}}{\left(I^{(2)}+1 / 2\right) g_{I}^{(2)}}=\frac{v_{a}^{(1)}}{v_{a}^{(2)}}
$$

Le rapport des déplacements est donc égal au rapport des fréquiences hyperfines. 
Pour les deux isotopes du rubidium, on a $v_{a}^{85} / v_{a}^{87}=3035,7 / 6834,7=$ 4,45. Nous avons mesuré, sur deux ensembles de cellules contenant du rubidium 85 , un déplacement linéaire de $235 \mathrm{~Hz} /$ torr dans le cas de l'azote et $177 \mathrm{~Hz} /$ torr dans le cas du néon. Les valeurs correspondantes relatives à ${ }^{87} \mathrm{Rb}$ sont $520 \mathrm{~Hz} /$ torr et $392 \mathrm{~Hz} /$ torr $\left[{ }^{54}\right)$ : on voit que la loi ci-dessus est bien vérifiée.

\section{2) Largeur de la résonance 0-0}

La mesure de la largeur atomique $\Delta u_{a}$ nous fournit le temps d'amortissement $T_{2}=1 / \pi \Delta \nu_{a}$ de l'élément non diagonal $\sigma_{+-}$sous l'action du pompage optique et des diverses causes de relaxation : on pourrait isoler l'effet de ces dernières en extrapolant les mesures de largeur pour des intensités lumineuses décroissantes et mettre en évidence l'influence de l'échange par. une étude en fonction de la température, comme nous l'avons fait ci-dessus pour l'observable $\langle\vec{S} . \vec{I}>$. Nous $n$ 'avons pas effectué de mesures systématiques, mais avons utilisé plusieurs fois la méthode pour mettre en évidence le rétrécissement de la résonance par réaction, comme nous le verrons plus loin. Donnons cependant un point de comparaison. Nous avons obtenu ci-dessus, pour la cellule contenant 6,3 torrs d'azote éclairée en géométrie d'illumination divergente et maintenue à $38,5^{\circ} \mathrm{C}:$

$$
\begin{aligned}
& T_{p m}^{-1} \text { (pompage) }=44 \mathrm{~s}^{-1} \\
& T_{H}^{-1} \text { (parois et gaz) }=25 \mathrm{~s}^{-1} \\
& T_{\mathrm{eH}}^{-1} \text { (Écinainge) }=30 \mathrm{~s}^{-1} \\
& \tau_{p}^{-1}=T_{P}^{-1}+T_{H}^{-1}+T_{e H}^{-1}=99 \mathrm{~s}^{-1} \text {, doù } \tau_{P} \simeq 10^{-2} \mathrm{~s}
\end{aligned}
$$

Dans les mêmes conditions, nous avons mesuré $\Delta v_{a}=74,5 \mathrm{~Hz}$, d'où $T_{2}^{-1}=234 \mathrm{~s}^{-1}$ et $T_{2}=4,27 \cdot 10^{-3} \mathrm{~s}$. 
D) ESSAI D'OBSERVATION DES MODULATIONS LUMINEUSES AUX FREQUENCES HYPERFINES

Nous avons vu au chapitre II que si le faisceau de pompage était polarisé circulairement, la lumière transmise par une celiule soumise à la résonance 0-0 devait être modulée à la fréquence va" Nous avons tenté sans succès de mettre cette modulation en évidence en employant comme détecteur un phototube à ondes progressives $\left({ }^{4}\right)$. L'échec est très simplement attribuable à un flux lumineux trop faible: la photocathode de ce tube ne mesure en effet que $2.5 \mathrm{~mm}^{2}$ et est vue de 1 'extérieur sous un angle solide très petit. Nous n'avons pu y concentrer que le centième du flux lumineux ayant traversé la cellule; le signal correspondant est alors noyé dans le bruit, malgré l'emploi du même récepteur très sensible que nous avons décrit. L'expérience sera reprise dès que nous disposerons d'un phototube plus utilisable. Rappelons qu'une expérience voisine, utilisant la transition 1-1, a été effectuée dans un autre laboratoire sur ${ }^{39} \mathrm{~K}$, dont la fréquence hyperfine plus basse $\left(v_{a}=\right.$ $462 \mathrm{MHz}$ permet l'emploi de celiules photoélectriques classiques beaucoup plus faciles à éclairer $\left({ }^{18}\right)$.

\section{E) AMPLIFICATION MASER PAR LA TRANSITION D-O}

Comme nous l'avons dit, l'étude radioélectrique ne nous apportera que peu de renseignements supplémentaires sur le système atamique lui-même; son principal intérêt sera de permettre la recherche des conditions d'oscillation, en jouant sur les différents facteurs à notre disposition pour essayer d'optimiser la valeur du paramètre d'amplification $p$. Nous en profiterons pour simplifier la représentation des résultats. Ainsi, lors du tracé des courbes relatives à l'amplification, nous porterons souvent en ordonnées non la valeur de $p$ ou de $G_{0}$, mais la variation $\Delta V_{0}=$ $V_{0}(p)-V_{0}(0)$ de la tension $V$ lue sur le voltmètre incorporé au récepteur lorsqu'on 
supprime l'amplification dans les conditions de l'expérience (en coupant par exemple le faisceau lumineux). Nous avons vu au chapitre précédent que $V$ était sensiblement proportionnel à la racine carrée de la puissance reçue et nous avons calculé, au chapitre III, la relation liant le gain (c'est-à-dire la puissance reçue à puissance incidente constante) au paramètre d'amplification $p$ : pour toutes les courbes présentées dans ce qui suit, nous avons choisi les conditions expérimentales let en particulier le couplage) pour que l'ordonnée $\Delta V_{0}$ soit une fonction monotone croissante de $p$ et avons éventuellement déterminé, par une mesure précise du gain, la valeur de p correspondant à la valeur maximum de $\Delta V_{0}$.

Chacune des mesures ci-dessous a, en général, été effectuée sur plusieurs cellules; nous présentons à chaque fois le résultat le plus caractéristique ou le plus complet.

\section{1) Influence du champ magnétique statique}

Nous avons vu que le champ directeur $\vec{H}_{0}$ était créé par trois jeux perpendiculaires de bobines d'Helmholtz. Nous avons réglé l'orientation de $\vec{H}_{0}$ de manière à ce qu'il soit parallèle à l'axe de la cavité et contrôlions cette orientation avant chaque expérience.

Comme on peut le vérifier sur la figure 1, le spectre des résonances hyperfines $\left(\Delta F=1, \Delta \mu_{F}=0, \pm 1\right)$ de l'état fondamental de ${ }^{85} \mathrm{Rb}(I=5 / 2)$ se compose au total de 11 raies distantes les unes des autres de $\nu_{Z}=g_{F} \mu_{B} H_{0} / h$, soit $v_{Z} / H_{0}=$ $467 \mathrm{kHz} / g a u s s$. La fréquence de la résonance $0-0$ ne dépend du champ qu'au second ordre suivant la loi $\nu_{a}(\mathrm{~Hz})=\left(\nu_{a}\right)_{H_{0}=0}+1290 \mathrm{H}_{0}{ }^{2}$ (gauss $\left.{ }^{2}\right)$. La largeur de cette résonance est donc en particulier peu sensible aux inhomogénéités de $\vec{H}_{0}$; celles-ci, de l'ordre de 10 milligauss sur notre montage, confèrent par contre aux autres raies une largeur de plusieurs kilohertz. Ces inhomogénéités sont surtout liées à la répartition des 
masses magnétiques autour du montage et subsistent en l'absence de tout champ appliqué. Si l'on diminue celui-ci jusqu'à une valeır inférieure à 10 milligauss, les différentes raies vont se chevaucher partiellement : nous avons alors constaté que le signal d'amplification disparait, contrairement à ce qui se produit dans le cas d'un maser à ${ }^{87} \mathrm{Rb}$ fonctionnant à l'intérieur d'un blindage magnétique $\left({ }^{55}\right.$ joù, au contraire, le signal augmente lorsque les raies ne sont plus résolues. Ceci se comprend si nous nous souvenons que le paramètre p est proportionnel à la quantíté $\alpha / \Delta u_{a}$. Lorsque les raies viennent à se confondre, I'inversion de populations totale augmente, puisque les sousniveaux $\mid F_{+}, \mu_{F}>y$ participent cette fois aux côtés du sous-niveau $\mid F_{+}, 0>$. Si la largeur $\Delta \nu_{a}$ reste sensiblement la même. le paramètre p doit alors augmenter : c'est le cas du maser fonctionnant dans un blindage où les inhomogénéités du champ statique ont une valeur très faible. Par contre, dans notre cas, la largeur effective $\Delta v_{a}$ augmente alors beaucoup puisque des composantes très elargies participent cette fois à la transition : il en résulte que p peut au total diminuer, comme nous l'avons observé. Au-delà de 10 milligauss, l'amplification est sensiblement indépendante du champ jusqu'à une valeur de quelques gauss.. Nous avons mené toutes nos expériences dans un champ appliqué de 50 milligauss, ce qui correspond à un déplacement d'environ $3 \mathrm{~Hz}$ pour la résonance $0-0$.

\section{2) Déplacement de la résonance 0-0 sous l'effet de la lumière}

Comme il est bien connu, l'action de la lumière de pompage provoque un déplacement de la fréquence de la résonance 0-0 proportionnei à l'intensité lumineuse. La grandeur et le signe de cet effet dépendent dans notre cas de nombreux facteurs que nous n'avons pas cherché à démêler. La figure 19 montre un exemple d'une telle variation; le déplacement maximum est de $24 \mathrm{~Hz}$, donc très notable par rapport à la largeur de raie qui valait $60 \mathrm{~Hz}$ dans ces conditions. La dispersion relativement grande des points ex- 


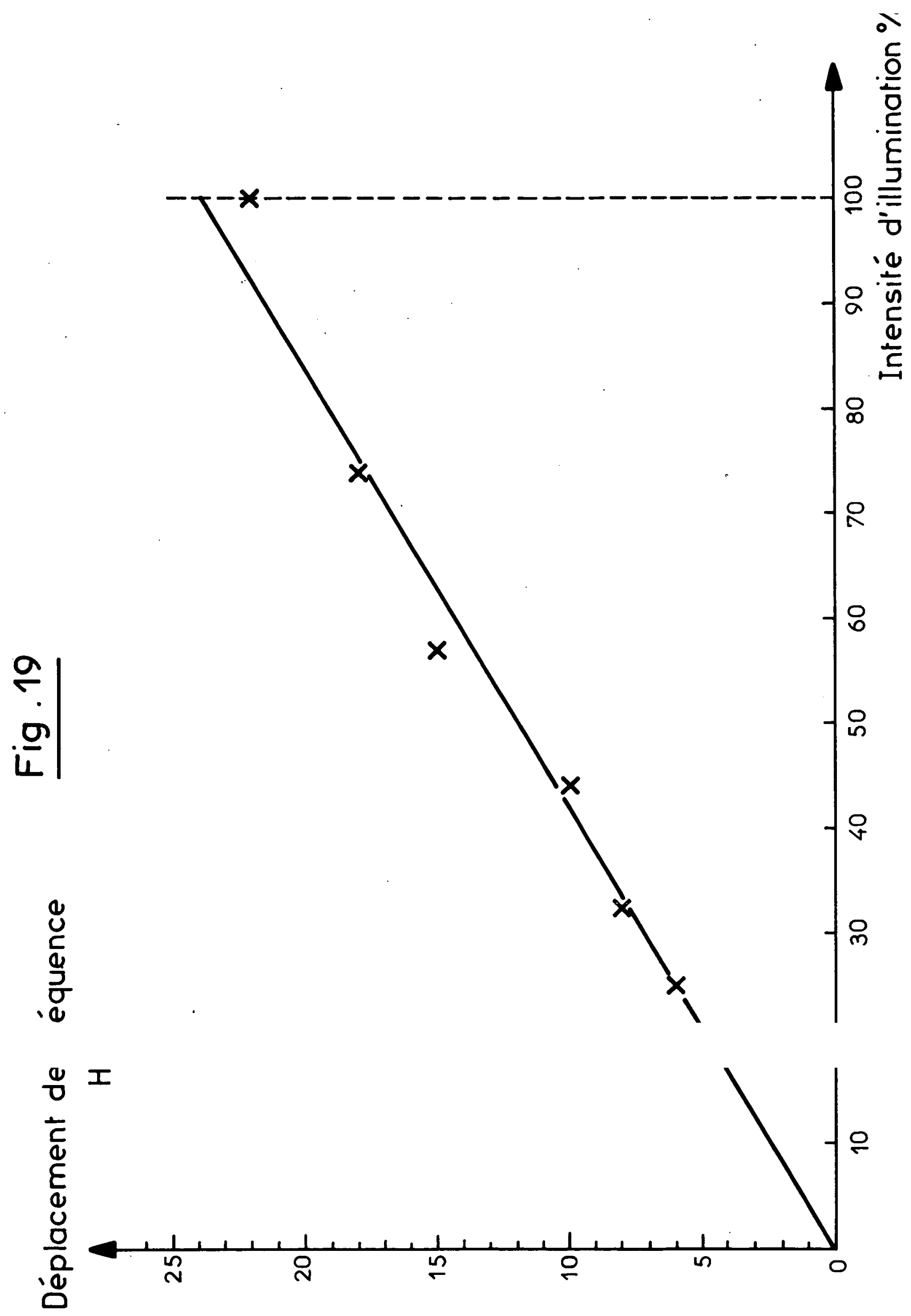




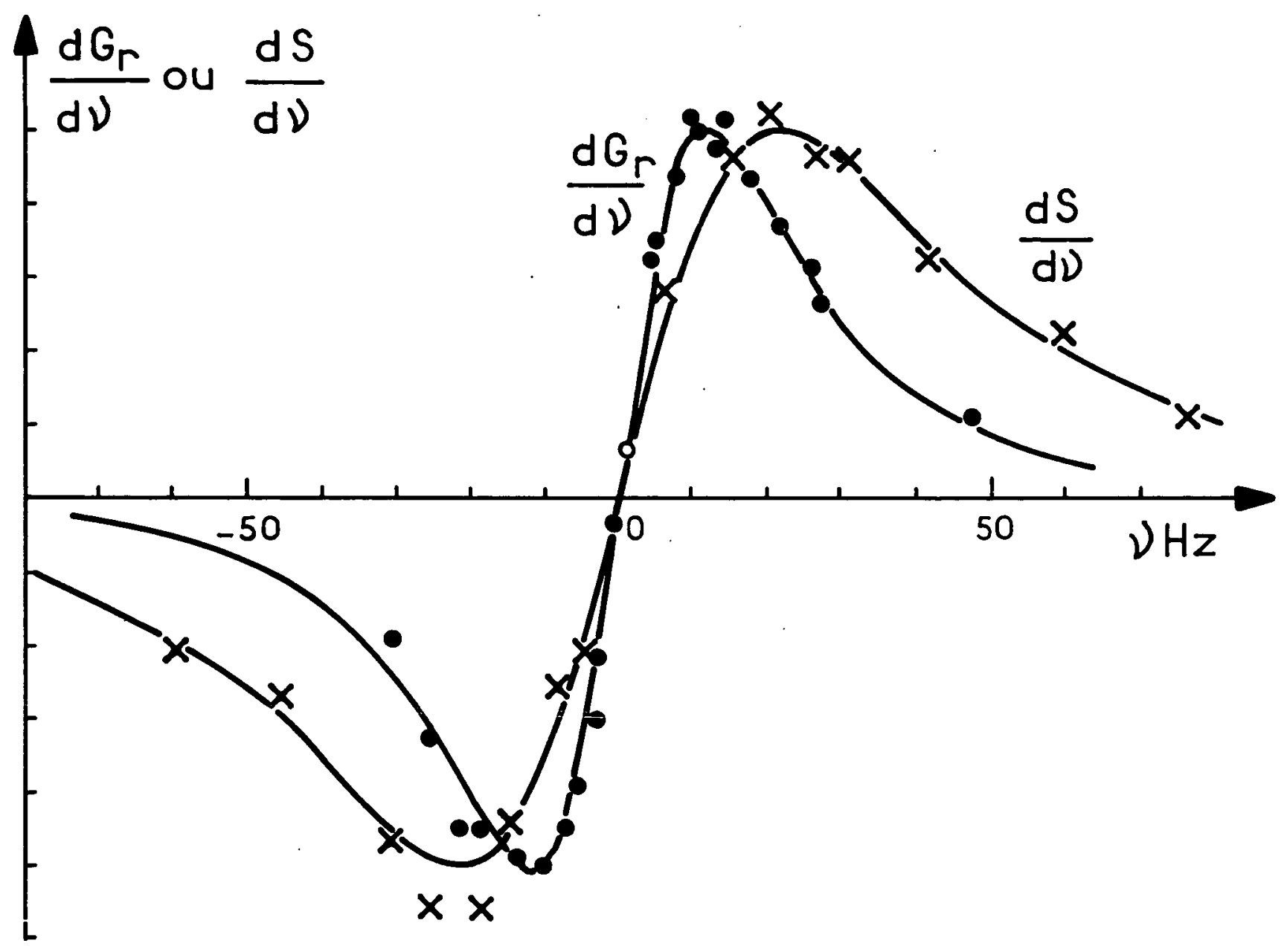

Fig. 20 
périmentaux provient de ce que la cellule était placée dans une cavité à forte surtension, dont de petits désaccords provoquent un entraînement de fréquence important : il serait plus précis d'utiliser une cavité à faible surtension et d'employer la détection optique.

\section{3) Rétrécissement de raie par réaction}

Au début de nos études sur l'amplification, la détermination de p à partir de la mesure du gain était peu précise car l'amplification était très petite. Nous avons alors assez systématiquement déduit p du rétrécissement par réaction de la résonance, conformément à la formule (22) du chapitre III. Pour cela, nous mesurions d'abord la largeur de raie avec une cavité très désaccordée; nous utilisions l'identité, démontrée au chapitre III, des résultats obtenus par détection radioélectrique et détection optique pour opérer le plus souvent avec cette dernière méthode, plus commode dans ces conditions. Nous accordions ensuite la cavité et mesurions à nouveau la largeur par détection radioélectrique, après revérification de la non-saturation. La figure 20 montre un exemple d'une telle mesure. En abscisse est portée la fréquence, en ordonnée la tension de sortie de la détection synchrone attaquée soit par le signal S issu du photodétecteur, soit par le niveau "vidéo" issu du récepteur dont les variations sont sensiblement proportionnelles ici aux variations correspondantes de $G_{r}$ : la détection synchrone fournit la dérivée de ces deux signaux. Les points sont expérimentaux, tandis que les courbes sont des dérivées de lorentziennes, $y=a x /\left(x^{2}+a^{2}\right)$ ajustées en un point : on voit que la cö̈ncidence est alors bonne pour tous les autres. L'échelle des abscisses illustre bien la stabilité de fréquence que doit posséder la source excitant la transition : l'incertitude sur la position des points expérimentaux est ici de $\pm 1 \mathrm{~Hz}$, ce qui correspond à $\Delta \nu / \nu \simeq 3.10^{-10}$. Nous lisons sur la figure 20 un rétrécissement par réaction d'environ $(21,5-12) / 21,5=44 \%$, ce qui correspond, avec les couplages employés 
$\left(\beta_{e}=0,01, \beta_{s}=0\right)$ à $p=0,445$. La mesure directe du gain dans les mêmes conditions nous a donné $G_{\text {or }}=0,93 \pm 0,02$. On voit sur la figure 6 que l'accord est bon, la détermination de p par le rétrécissement de la raie étant d'ailleurs d'une précision bien supérieure.

\section{4) Variation de l'amplification avec l'intensité d'illumination}

Nous avons étudié la variation de l'amplification avec l'intensité lumineuse, pour différentes valeurs de la température de la cellule (fig. 21) ou du filtre (fig. 22). Les deux réseaux ont une allure très semblable. Pour les températures de la cellule (ou du filtre) pas trop élevées, la courbe passe par un maximum. Nous avons vu en effet plus haut qu'une fois la saturation lumineuse atteinte (fig. 17), la différence de populations a ne pouvait pius augmenter; par contre, la diminution de $T_{p}$ entraîne une augmentation de la largeur de raie $\delta \omega_{a}$ et donc une diminution du paramètre d'amplification p. Aux températures élevées, ce maximum n’est pas atteint par suite de l'insuffisance du flux lumineux : nous avons effectivement vérifié qu'il était possible de l'observer à des températures supérieures en démontant l'obturateur qui diaphragmait partiellement le faisceau.

5) Variation de l'amplification avec la pression de gaz étranger dans la cellule

Nous avons pu disposer d'une série de cellules sphériques en silice identiques contenant $3,4,5,05,6,55,6,7$ et 8,9 torrs d'azote. Les résultats se sont révélés sensiblement indépendants de la pression dans les limites ci-dessus.

Nous avons également étudié des cellules sans gaz étranger. Elles donnent lieu à une amplification moins élevée; nous verrons à l'appendice II que ceci est dû à un coefficient de remplissage $n$ inférieur. 


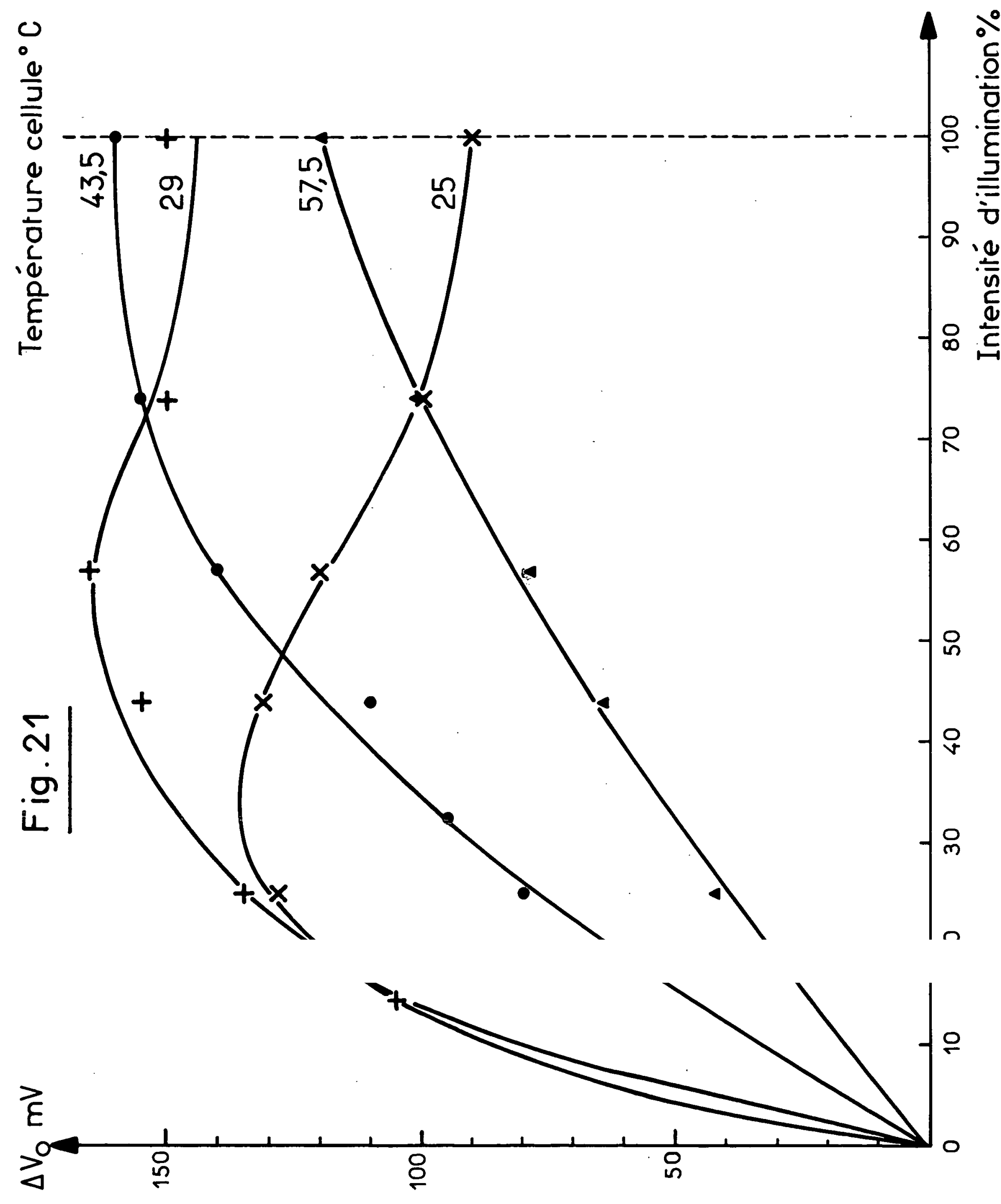




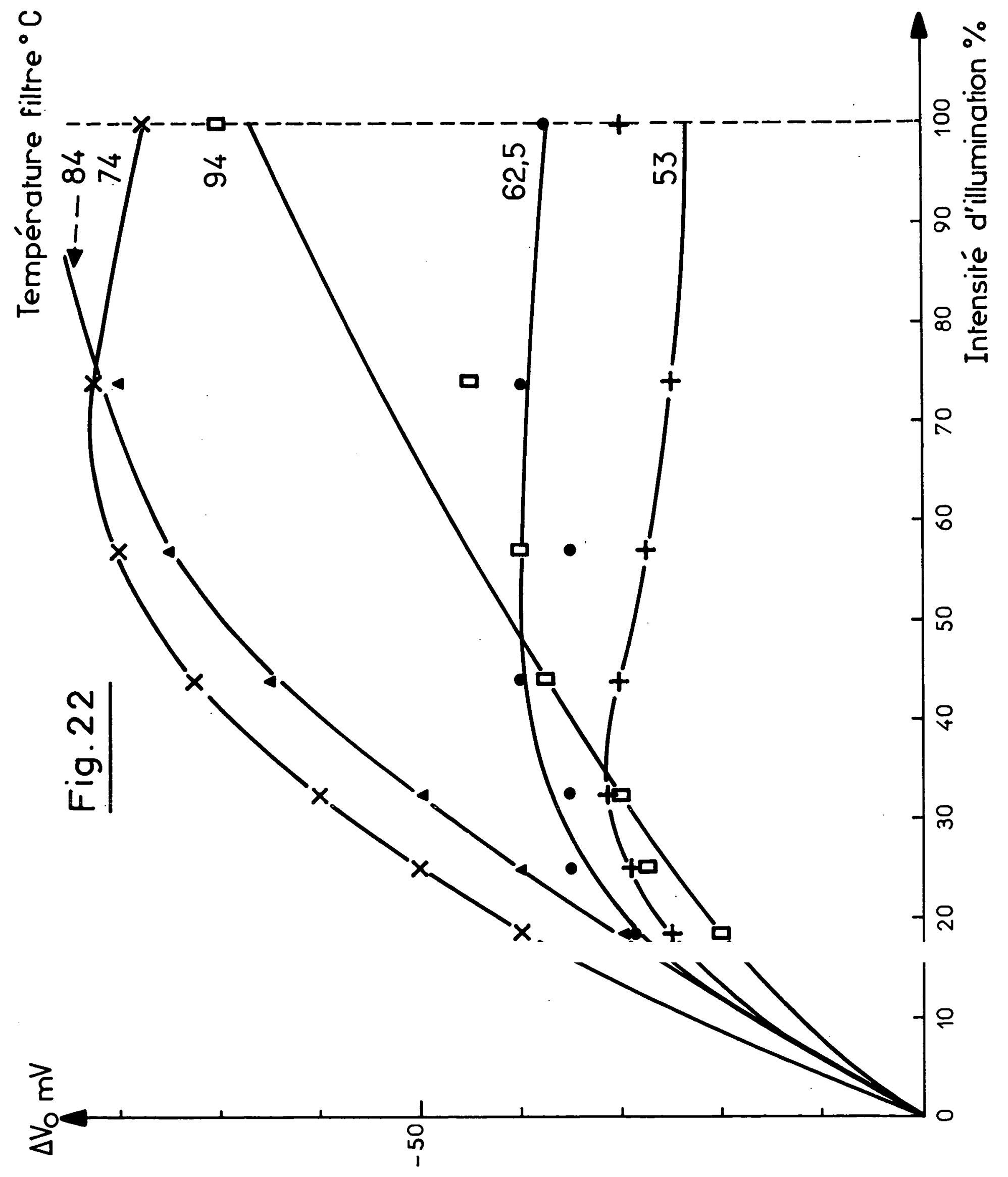




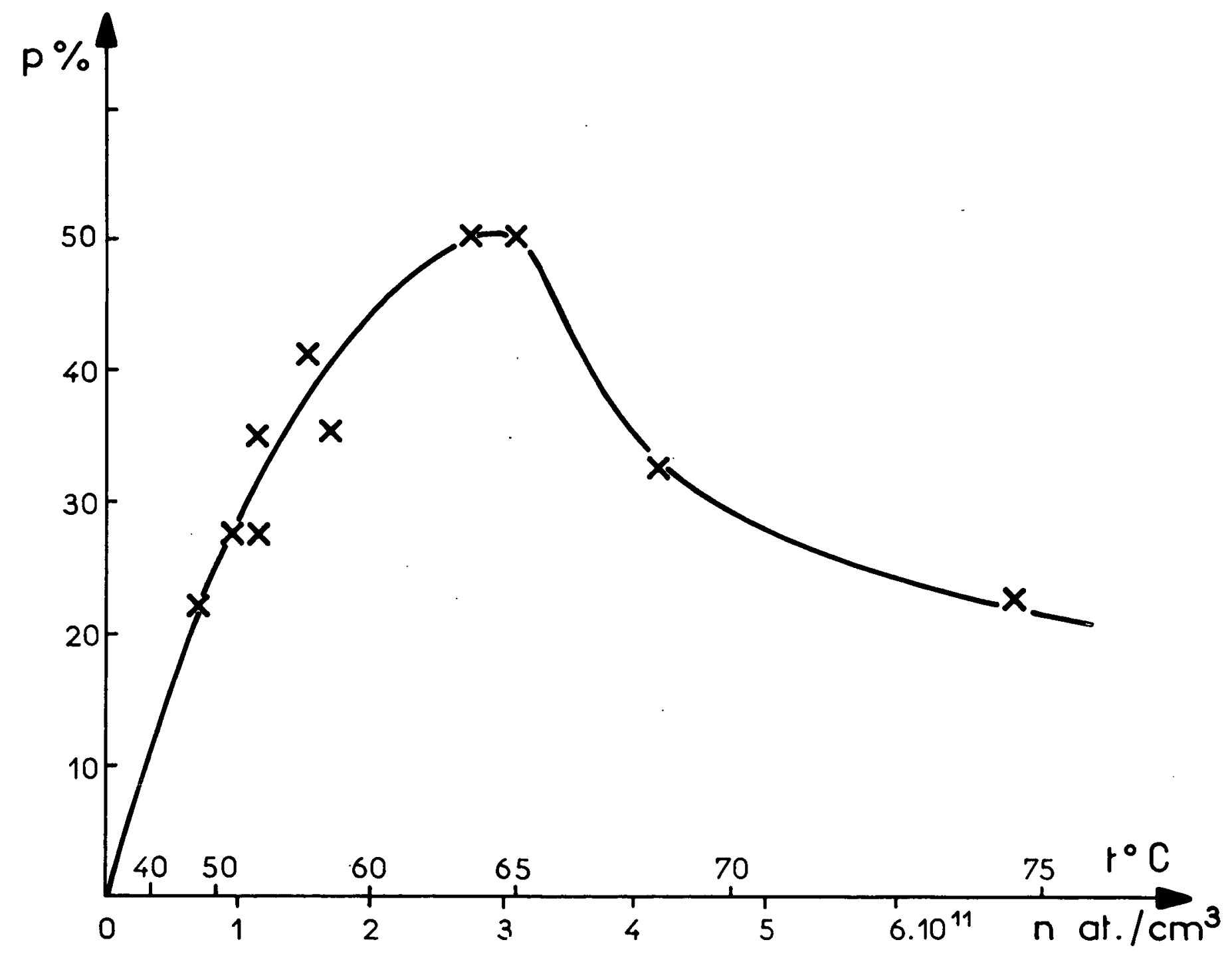

Fig. 23 
6) Variation de l'amplification avec la température de la cellule Nous avons cette fois calculé la valeur de p, porté sur la figure 23 en fonction de la température dans l'échelle déjà utilisée. Les mesures sont relatives à une cellule sphérique de configuration E, contenant 6,55 torrs d'azote. Lorsque la température de la cellule s'élève, le paramètre p commence par augmenter, par suite de l'accroissement de la densité $n$. Lorsque celle-ci devient suffisante pour que les collisions d'échange soient le mécanisme de relaxation dominant, la largeur $\delta \omega_{a}$ tend à devenir proportionnelle à $n$, et le rapport $n / \delta \omega_{a}$ ne dépend plus de $n$. Par ailleurs, lorsque cette densité atteint une valeur telle que l'absorption optique soit pratiquement totale, l'inversion de populations an ne peut plus augmenter et finit même par diminuer sous l'effet des collisions d'échange. De plus, les atomes effectivement soumis au pompage optique se trouvent alors de plus en plus confinés au voisinage de la face d'entrée, là où la lumière pénètre encore; le champ $\vec{H}$ étant très faible dans cette région, le coefficient de remplissage $n$ tend vers 0 . Le paramètre $p=K a n n Q_{0} / \delta \omega_{a}$ doit done passer par un maximum lorsque $n$ augmente, comme nous l'avons observé.

7) Variation de l'amplification avec les caractéristiques du filtre

Il existait dans le laboratoire une série de cellules sphériques de $6 \mathrm{~cm}$ de diamètre, contenant du rubidium 87 et de l'hélium ( ${ }^{3} \mathrm{He}$ et ${ }^{4} \mathrm{He}$ ) à différentes pressions; nous les avons utilisées comme filtres. La figure 24 montre les résultats obtenus pour ies filibes à ${ }^{3}$ He en fonction de la température : on voit que dans tous les cas, $\Delta V_{0}$ passe par un maximum. L'élimination, dans la lumière issue de la lampe, des composantes nuisibles est, en effet, d'autant mieux assurée que la température du filtre est plus élevée; cependant, comme nous l'avons signalé, le filtre absorbe aussi les composantes utiles et la quantité totale de lumière transmise, au-dessus d'une certaine température, devient insuffisante pour pomper efficacement la cellule. La température 
correspondant au maximum.de $\Delta V_{0}$ est d'autant plus élevée que la pression du gaz étranger dans le filtre est plus basse, mais la valeur de ce maximum en dépend peu. La série des filtres à ${ }^{4}$ He nous a donné des résultats très semblables.

Nous avons également pu étudier des filtres contenant du rubidium 87 à 99,995\% et un mélange d'environ 65 torrs d'hélium 4 et 10 torrs d'azote : la grande pureté de l'isotope doit réduire l'absorption indésirable par les traces de $85 \mathrm{Rb}$, qui peut ne pas être négligeable si le filtre est porté à une température élevée, tandis que l'azote doit supprimer la fluorescence nuisible, comme nous l'avons expliqué au chapitre précédent. La figure 25 représente la variation de l'amplification en fonction de la temperature d'un tel filtre employé avec un faisceau lumineux très auvert, cette fois pour différentes températures de la cellule. Le maximum s'explique comme précédemment : il se produit à une température du filtre d'autant plus élevée que celle de la cellule est basse, par suite de la moins grande quantité de lumière nécessaire pour atteindre la saturation lumineuse dans ce dernier cas. Ceci est à rapprocher de la variation de l'amplification avec l'intensité d'illumination que nous avons étudiée plus haut : nous avons vérifiéici que, lorsqu'on augmente l'intensité d'illumination, le maximum de $\Delta V_{0}$ relatif à un filtre et à une température de cellule donnés est plus élevé et se produit à une température du filtre plus élevée. La meilleure valeur que nous ayions pu obtenir, indiquée par une flèche sur la figuré, correspond à $p=0,85$. Il est ainsi intéressant de disposer d'une intensité d'illumination la plus élevée possible, quitte à la réduire en chauffant davantage le filtre, ce qui améliore en même temps la qualité du pompage. Par ailleurs, nous avons vu que la lumière pénètre mal dans la cellule lorsque la tension de vapeur de l'alcalin dans celle-ci est trop élevée. Ces deux raisons nous ont conduits à installer un deuxième faisceau d’illumination très ouvert identique au premier, éclairant la cellule du 


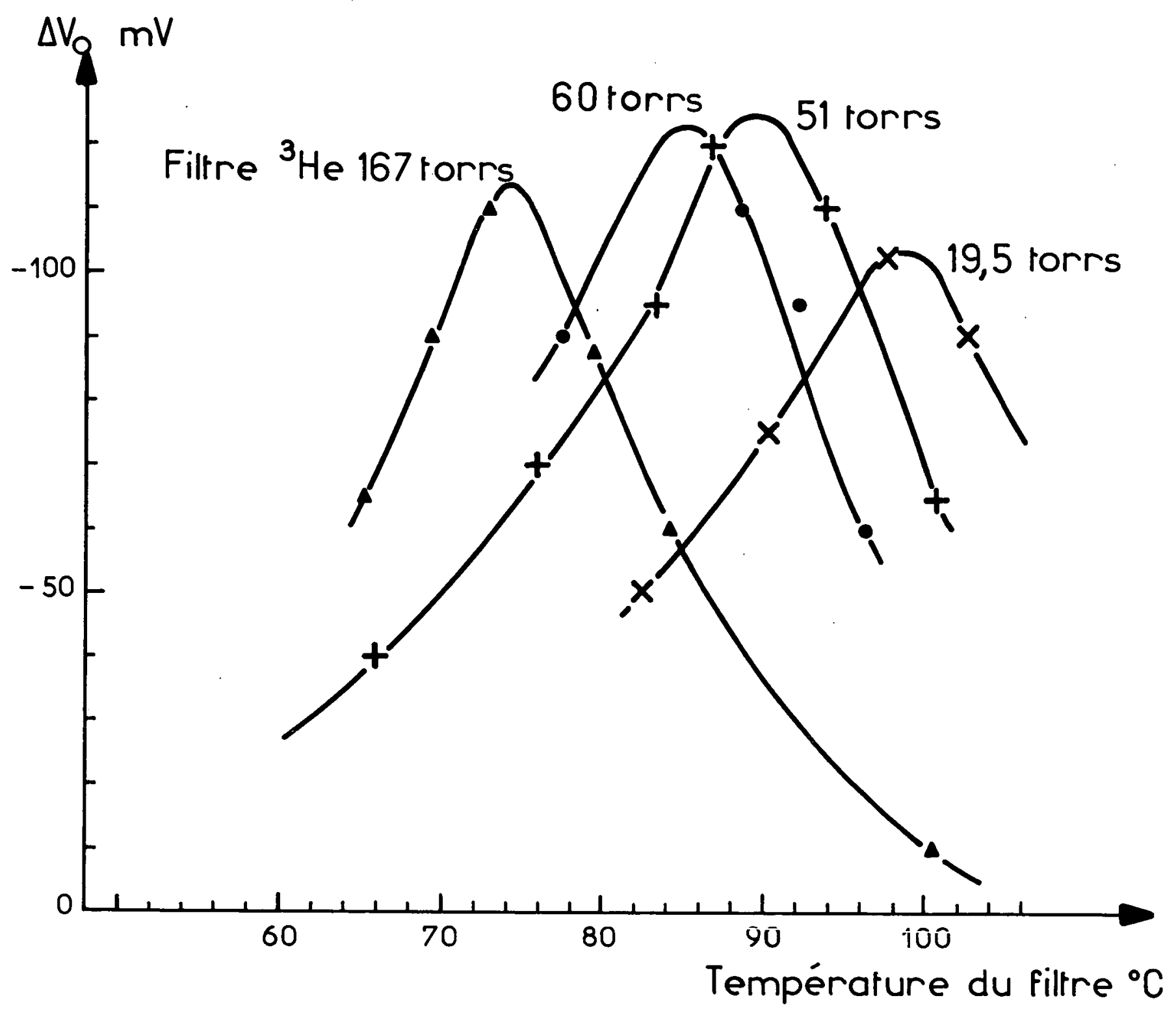

Fig. 24 


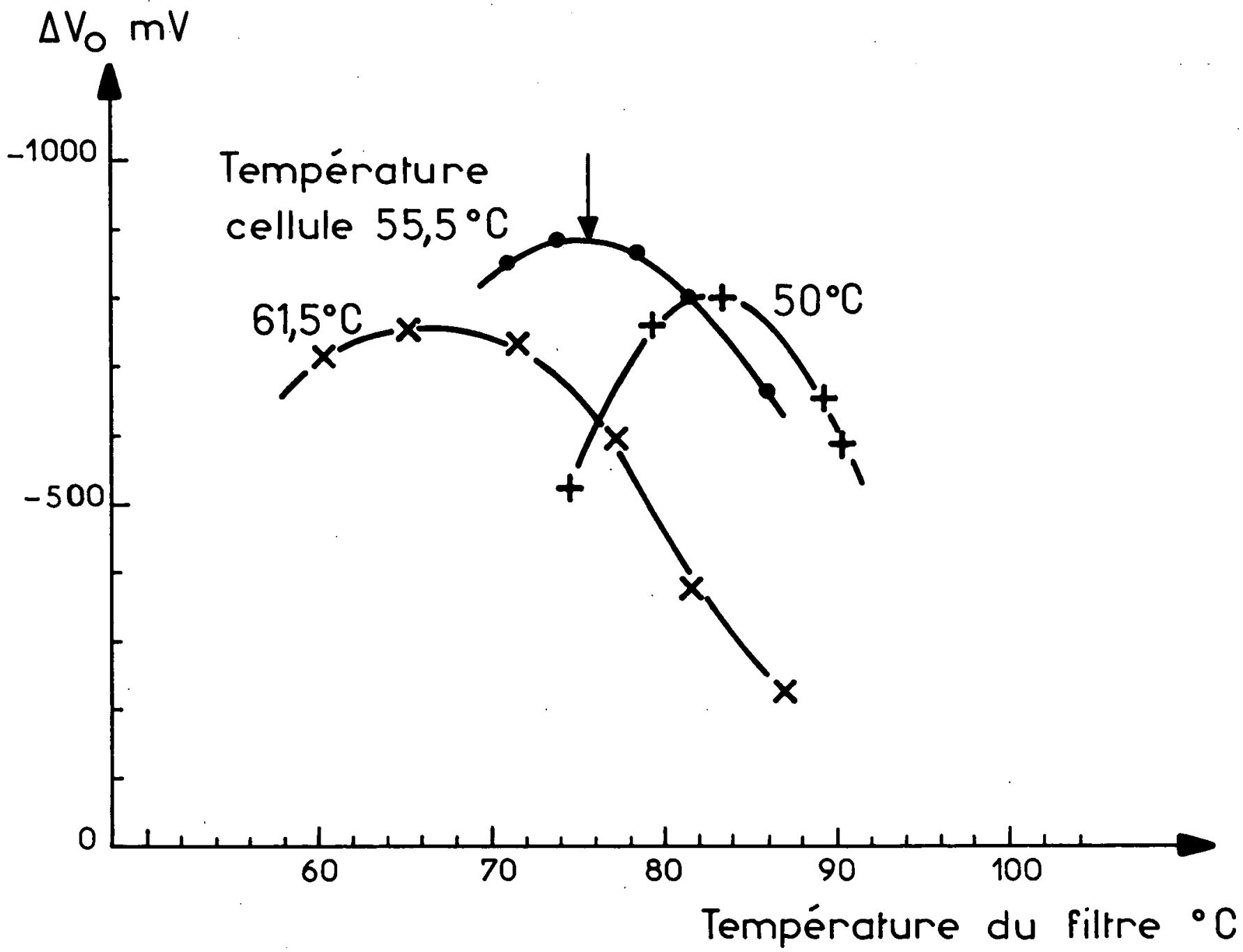

Fig. 25 


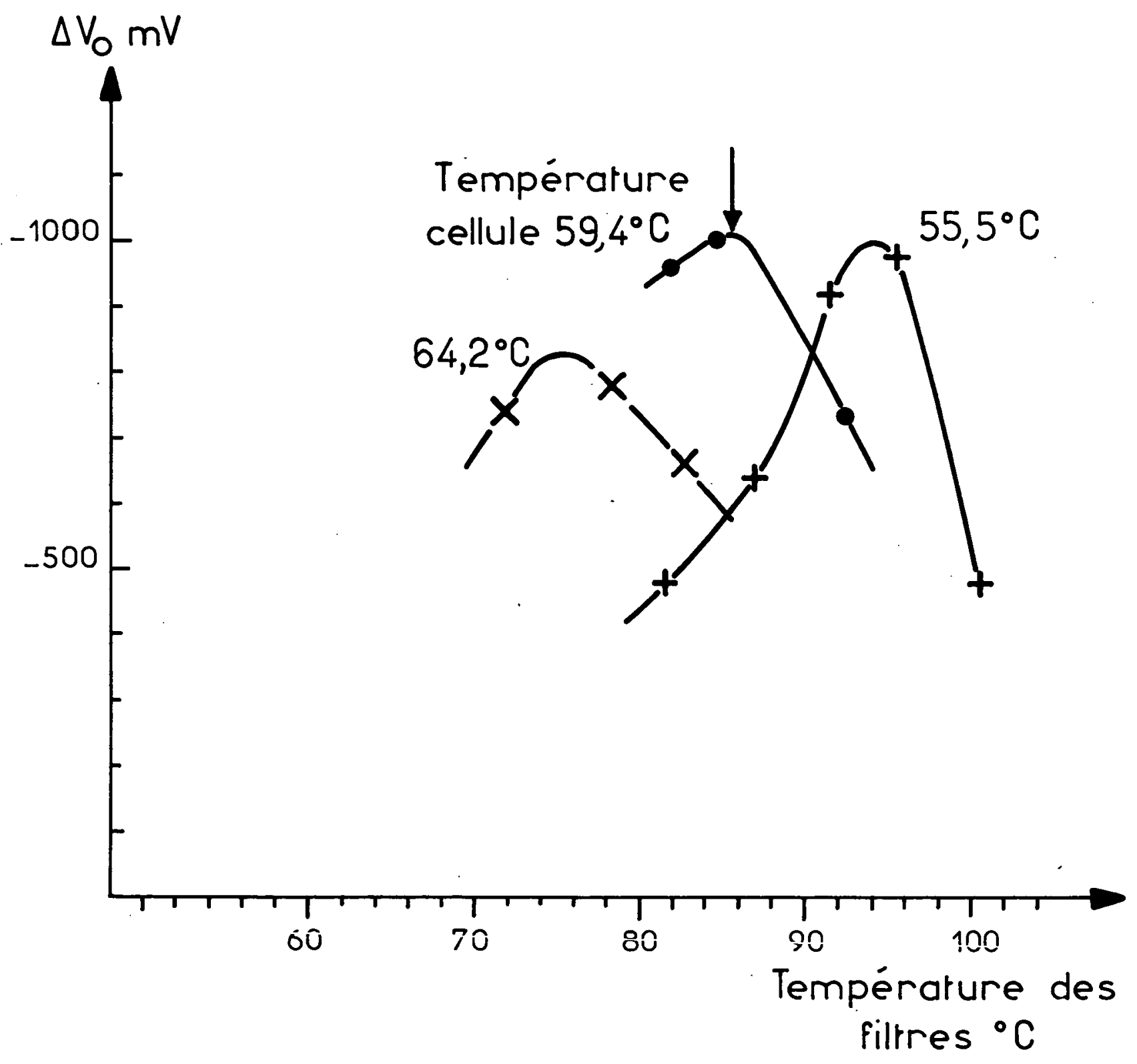

Fig. 26 
côté opposé. La figure 26 montre la variation de l'amplification en fonction de la température commune des deux filtres pour différentes températures de la cellule, les conditions expérimentales étant par ailleurs identiques à celles de la figure 25 . On voit que l'installation du deuxième faisceau, par le gain de lumière qu'il procure, a permis de chauffer les filtres davantage et que la valeur optimum de $\Delta V_{0}$ est plus élevée.

Cette valeur, indiquée par une flèche sur la figure, est la meilleure que nous ayions pu atteindre jusqu'à présent. Les conditions expérimentales détaillées étaient les suivantes :

- Configuration de l'ensemble cellule-cavité :

- Gaz étranger dans la cellule :

- Température de la cellule:

- Champ magnétique statique :

- Illumination :

- Structure des filtres:

- Température des filtres :
$F$ (sphérique), cellule paraffinée. Surtension : $Q_{0} \simeq 4 \cdot 10^{4}$. Couplages : $\beta_{e}=0,05, \beta_{s}=0$

Azote à 8,9 torrs

$59,4^{\circ} \mathrm{C}$

$50 \mathrm{mG}$ suivant l'axe de la cavité

Deux faisceaux symétriques très divergents épaisseur $36 \mathrm{~mm}$, contenant ${ }^{87} \mathrm{Rb}$ à $99,995 \%+$ 65 torrs de ${ }^{4} \mathrm{He}+10$ torrs de $\mathrm{N}_{2}$

$85.5^{\circ} \mathrm{C}$

La valeur de $\Delta V_{0}$ obtenu dans ces conditions correspond à $p=0,88$, c'està-dire à $88 \%$ du seuil d'oscillation, car il est toujours possible d'obtenir celle-ci en réduisant les couplages lorsque p excède la valeur 1. L'amélioration par rapport au cas de la figure précédente (un seul faisceau, $P=0,85$ ) ne semble pas considérable; d'autres configurations nous ont d'ailleurs donné des résultats comparables, allant de 0,72 à 0,86 . Peut-être la très grande finesse de la raie lorsqu'on s'approche ainsi du seuil d'oscillation introduit-elle une erreur systématique : bien que les instabilités de fréquence du générateur que nous utilisons soient très faibles, comme nous le verrons 
plus loin, elles peuvent au voisinage du seuil excéder la largeur de raie et limiter la valeur maximum de p mesurée. Une saturation partielle de la résonance devient également de plus en plus difficile à éviter. Nous n'avons cependant pas atteint les conditions d'accrochage, car aucun signal n'a pu être décelé en l'absence de l'onde incidente.

Nous pensons qu'il doit être possible d'obtenir l'oscillation en continuant à améliorer successivement les différents éléments et réglages du montage actuel. A la température et à la pression où s'observe la valeur de p maximum, les conditions physiques ne sont pas uniformes en tous les points de la cellule; l'étude de l'absorption optique en l'absence de radiofréquence ne fournit plus de résultats interprétables et il est difficile de relier la valeur moyenne de p mesurée à celle des différentes observables du système atomique. Nous pouvons cependant considérer que les conditions de l'expérience correspondaient grossièrement à $\alpha \simeq 6.10^{-2}, n \simeq 1,6.10^{11}$ atomes $/ \mathrm{cm}^{3}$, $2 \delta \omega_{a} / 2 \pi=\Delta \nu_{a} \simeq 60 \mathrm{~Hz}, \eta \simeq 0,5$ et $Q_{0} \simeq Q_{c} \simeq 4.10^{4}$, valeurs que nous avons montré, au chapitre $V$, conduire à $p \simeq 1$. Il semble que les efforts d'amélioration devraient d'abord porter sur la valeur de l'inversion de populations $\alpha$, par l'emploi de lampes plus intenses et de filtres plus efficaces.

\section{8) Régimes transitoires}

Nous avons signalé la richesse de ces méthodes qui permettent la détermination de nombreux temps de relaxation. Nous nous sommes jusqu'à présent bornés à mettre en évidence l'effet d'une brusque suppression de l'illumination. Notre formalisme ne nous permet pas de traiter ce cas en détail, puisque l'équation d'évolution de la matrice densité à l'ordre 1 a été obtenue en y remplaçant les populations par leur valeur d’équilibre à l'ordre 0 . Nous pouvons cependant prévoir que la suppression de l'il. lumination élimine l'effet du temps de pompage $T_{p}$ sur la relaxation : la largeur atomi- 


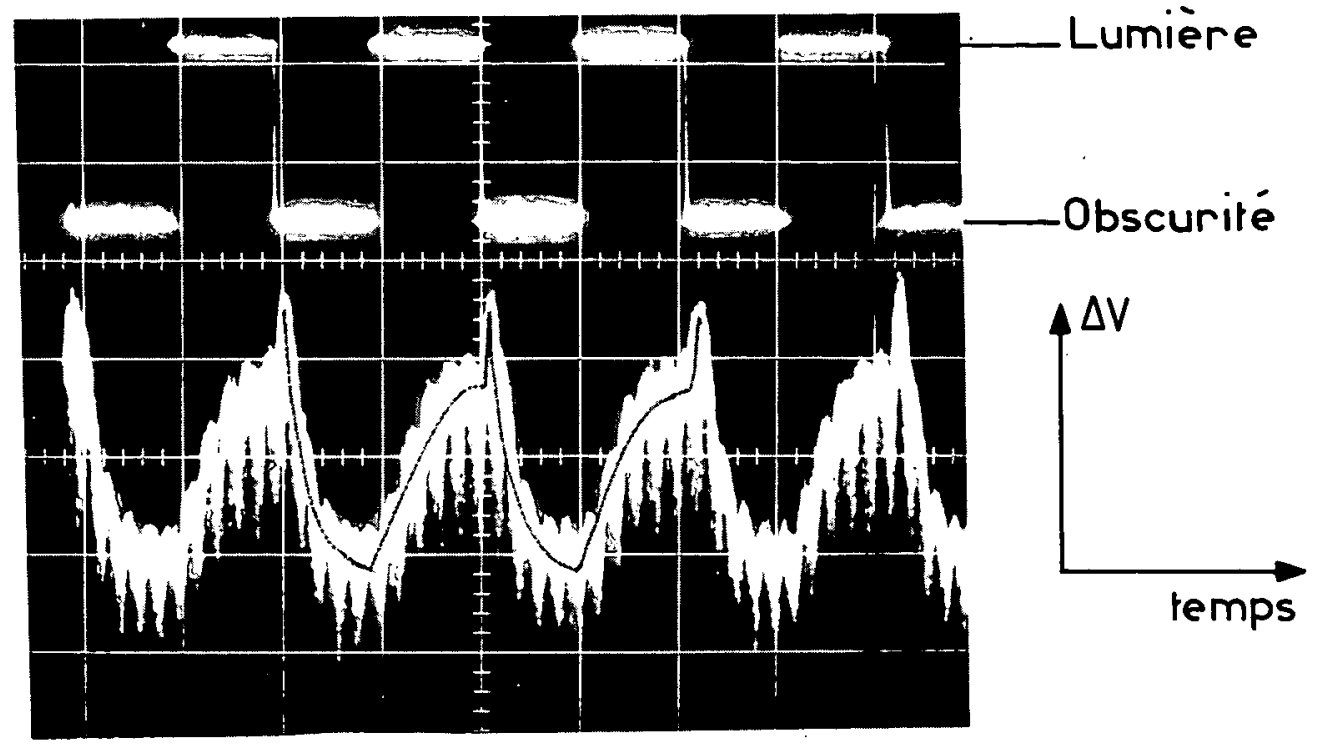

Fig. 27 
que $\delta \omega_{a}$ doit donc brusquement diminuer, tandis que l'inversion de populations subsiste pendant un temps de l'ordre de $T_{1 H}$ : la valeur de $p$, et donc l'amplification, doit donc brusquement augmenter avant de décroitre sous l'effet de la relaxation. Ce phénomène, déjà observé dans le cas du maser à ${ }^{87} \mathrm{Rb}\left({ }^{4}\right)\left({ }^{38}\right)$, est visible sur la photographie de la figure 27 (la trace inférieure est perturbée par des impulsions parasites à $100 \mathrm{~Hz}$.

\section{F) ETUDE DE L'OSCILLATION AVEC REACTION EXTERIEURE}

Nous avons vu au chapitre IV qu'en présence d'une réaction extérieure de gain global G', la condition d'autooscillation prenait la forme :

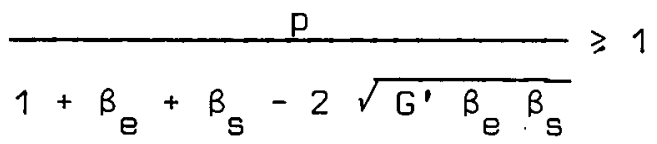

Cette condition est naturellement plus facile à satisfaire qu'en l'absence de réaction extérieure, lorsque $G^{\prime}=0$. Nous avons ainsi pu réaliser un oscillateur avec une cavité pour laquelle le paramètre d'amplification p valait seulement 0,35 , munie de deux couplages $\beta_{e}=0,15$ et $\beta_{s}=0,55$. La figure 28 indique la variation de la puịsance $P_{r}$ recueillie à la sortie du circulateur en fanction du réglage de l'atténuateur, c'est-à-dire du gain G' dans la boucle de réaction. La puissance maximum atteint $10^{-8}$ watt.', supérieure donc à la puissance obtenue jusqu'à présent pour

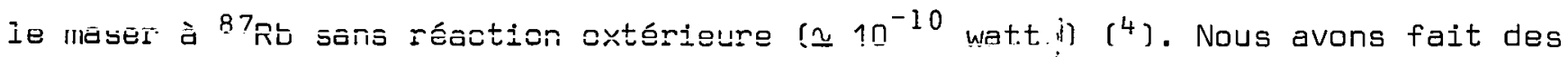
mesures préliminaires de la stabilité de fréquence en envoyant simultanément dans le récepteur la puissance $P_{r}$ à la fréquence $v$ et un signal issu du générateur hyperfréquence réglé à une fréquence très légèrement différente v'; à la sortie "vidéo" du récepteur, on recueille une basse fréquence $\left|\nu-v^{\prime}\right|$ dont les variations mesurent directement l'instabilité des deux oscillateurs l'un par rapport à l'autre. On 
peut étudier $\left|v-v^{\prime}\right|$ sur un périodemètre; mais il est préférable d’observer la courbe de Lissajous formée entre $\left|v-v^{\prime}\right|$ et un oscillateur de référence dont on s'assure aisément que la stabilité n'a pas besoin d'être très élevée. L'ellipse formée reste stable (rotation inférieure au dixième de tour) pendant des temps de plusieurs secondes : sur cet intervalle, les fréquences $v$ et v' varient donc l'une par rapport à l'autre de moins de $0,1 \mathrm{~Hz}$, ce qui correspond, pour $v_{a}=3035,7 \mathrm{MHz}$, à une instabilité relative inférieure à $3 \cdot 10^{-11}$ sur une seconde $\left({ }^{8}\right)$.

Le quartz de base attaquant la chaîne multiplicatrice ayant, d’après le constructeur, une stabilité nominale de $5.10^{-11}$ par seconde, l'instabilité observée est sans doute due principalement au générateur hyperfréquence utilisé dans la comparaison. Nous avons actuellement commencé la construction d'un second maser à réaction extérieure identique au précédent afin de pouvoir effectuer une étude plus poussée de la stabilité. Par rapport à un maser sans réaction extérieure, la présente solution a, actuellement, I'avantage d'une puissance de sortie plus élevée, ce qui est favorable à une bonne stabilité, comme nous l'avoris vu au chapitre IV.Il resteraie toutefois à s'assurer que le bruit supplémentaire introduit par l'amplificateur extérieur ne détruit pas cetsavantage (2?) a rappelons que l'amplificateur à diode tunnel que nous avons utilisé présentait un facteur de bruit de $5,5 \mathrm{db}$. 


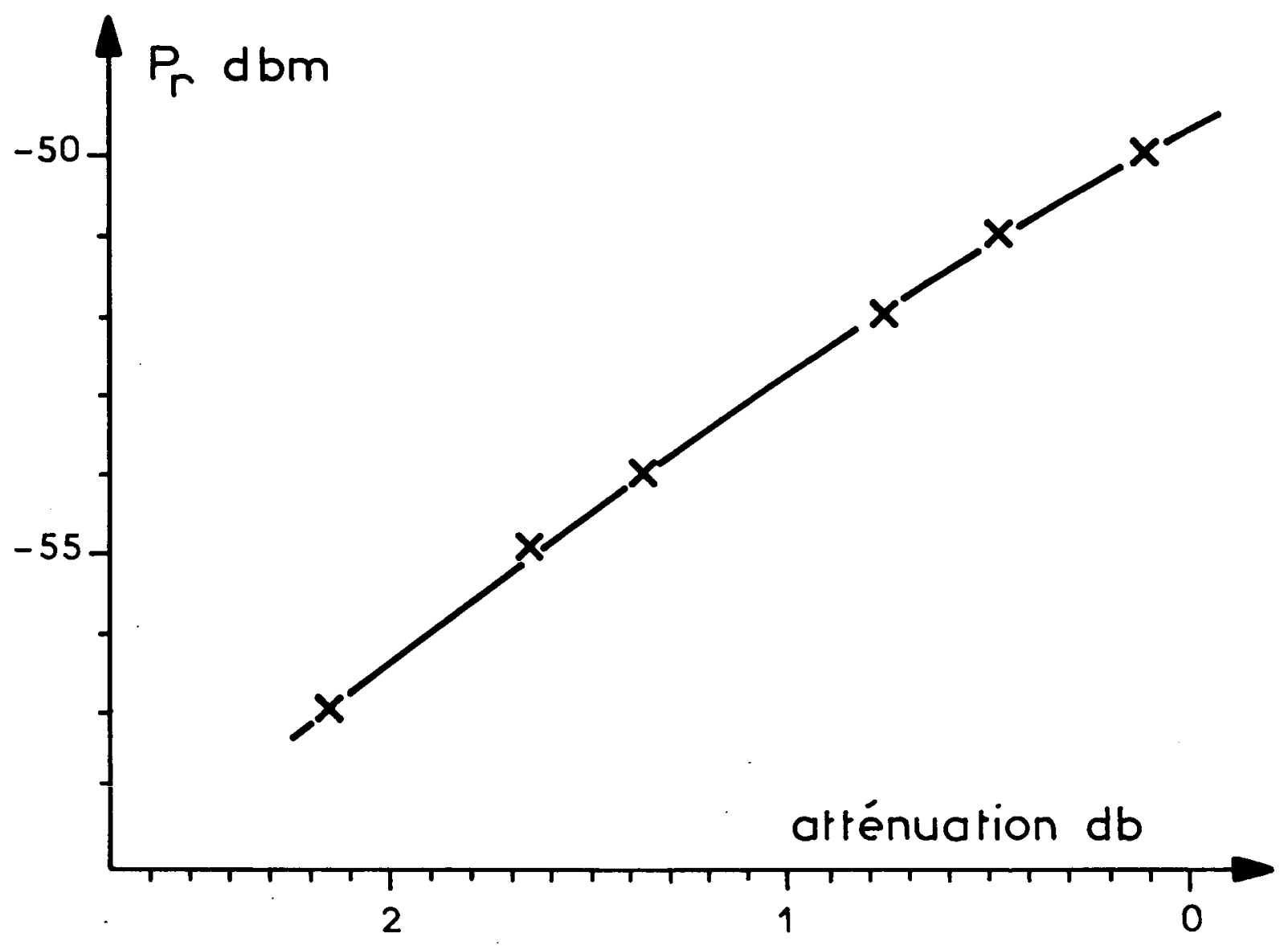

Fig. 28 
Le but que nous nous étions proposés au début de notre travail: obtention de l'autooscillation maser de la vapeur de rubidium 85 pompé optiquement, n'a pas encore été pleinement atteint, par suite des nombreuses difficultés expérimentales que nous avons rencontrées. En vue de mieux comprendre les phénomènes dont le système atomique est le siège, nous avons été amenés à mettre en oeuvre différentes méthodes d'observation et, en particulier, à effectuer une étude détaillée de l'amplification maser. Nous avons ainsi établi un ensemble de relations décrivant de manière très générale le comportement radioélectrique d'une cavité renfermant un système atomique résonnant, dans le cas où la largeur de bande de la cavité est très supérieure à celle de la transition atomique. Appliquées au cas de la résonance $0-0$ de ${ }^{85} \mathrm{Rb}$, ces relations sont bien vérifiées par l'expérience et lui servent de guide dans la recherche des conditions d'oscillation; les mesures d'absorption optique, dont l'interprétation est plus sûre, constituent d'ailleurs un complément indispensable des mesures d'amplification radioélectrique. A la suite de continuelies améliorations, nous sommes actuellement parvenus à $88 \%$ au moins du seuil d'oscillation, qu'il doit être possible d'atteindre en continuant l'optimisation du montage actuel. En insérant la cavité renfermant le système atamique dans une boucle de réaction extérieure comprenant un amplificateur, nous avons pu d'autre part réaliser un oscillateur dont les propriétés (puissance $10^{-8}$ watt; instabilité de fréquence inférieure, d'après les mesures préliminaires, à $3.10^{-11}$ sur une seconde) semblent également très intéressantes et qui, comme le maser proprament dit, paraît susceptible d'être construit sous un faible volume $\left(11 \mathrm{dm}^{3}\right)\left({ }^{56}\right)$. Notre travail apparaît ainsi comme une éta$\ldots / \ldots$ 
pe et doit être prolongé dans les directions suivantes :

- Poursuite des efforts en vue d'obtenir l'autooscillation sur le montage actuel par amélioration des différents éléments et réglages, en particulier des lampes, du filtre et de la cavité.

- Mise au point de nouvelles méthodes d'observation du système atomique, parmi lesquelles l'étude des régimes transitoires et celle des modulations lumineuses aux fréquences hyperfines.

- Etude plus poussée des performances de l'oscillateur à réaction extérieure et détermination de sa stabilité de fréquence intrinsèque.

- Essai de nouveaux procédés de pompage optique, éventuel lement applicables à d'autres alcalins, tel que le pompage hyperfin du césium par la lumière d'un laser à semiconducteur.

Les nombreuses applications, mentionnées brièvement dans l'introduction, auxquelles la stabilité de fréquence à court terme du maser ou de l'oscillateur à réaction extérieure doit donner lieu justifient la poursuite et la diversification des recherches sur ce sujet malgré les grandes difficultés expérimentales qui restent à résoudre. 


\section{A P PENDICE I \\ CARTE DU MODE TE 011 \\ 00000000000000000000}

En vue de la réalisation d’ondemètres (cavités étalon à haute surtension) les modes d'une cavité cylindique ont été très étudiés. Il existe des abaques donnant les fréquences de résonance en fonction des dimensions du cylindre; les équations des modes ont été établies $\left({ }^{57}\right)$ ainsi que, pour les principaux d'entre eux, la carte des courants dans les perois et la relation liant la surtension à la conductivité de celles-ci (58). Pour les raisons exposées au chapitre $V$, nous avans choisi d'utiliser dans nos expériences Ie mode TE 011. D et $L$ désignant respectivement le diamètre et la longueur du cylindre, la fréquence de résonance $v$ est donnée par :

$$
(v D)^{2}=\left(\frac{c}{\pi} u_{01}^{\prime}\right)^{2}+\left(\frac{c}{2}\right)^{2}\left(\frac{D}{L}\right)^{2}
$$

où c est la vitesse de la lumière, $U^{\prime}{ }_{01}=3,83171$ le premier zéro de la fonction de Bessel $J_{l}$; la constante diélectrique $\varepsilon$ a été prise égale à 1.

La symétrie du problème impose un système de coordonnées cylindriques (fig. 29). En prenant l'origine 0 au centre d'une des bases du cylindre, les équations des champs s'écrivent :

$$
\begin{aligned}
& E_{\rho}=0 \\
& E_{\phi}=-J{ }_{0}\left(k_{1} \rho\right) \sin k_{3} z \\
& E_{z}=0
\end{aligned}
$$




$$
\begin{aligned}
& H_{\rho}=\frac{k_{3}}{k} J_{0}^{\prime}\left(k_{1} \rho\right) \cos k_{3} z \\
& H_{\phi}=0 \\
& H_{z}=\frac{k_{1}}{k} J_{0}\left(k_{1} \rho\right) \sin k_{3} z
\end{aligned}
$$

avec $k_{1}=2 u^{\prime}{ }_{01} / 0, k_{3}=\pi / L, k^{2}=k_{1}^{2}+k_{3}^{2} ; J_{0}$ est la fonction de Bessel d'ordre 0 , de dérivée $J^{\prime} 0=-J_{1}$. On a par ailleurs $\lambda=c / v=2 \pi / k$.

L'épaisseur de peau correspondant à la conductivité de la parai étant désignée par $\delta$, la surtension à vide est dannée par la formule générale :

$$
Q_{0}=\frac{2}{\delta} \frac{\iiint_{\operatorname{cav}} H^{2} d V}{\iint_{\text {paroi }} H^{2} d S}
$$

Connaissant la configuration d'un mode, on peut calculer les intégrales cidessus. On trouve que, pour le mode TE 011, la quantité $Q_{0} \delta / \lambda$ passe par un maximum égal à 0,66 lorsque $\mathrm{D} / \mathrm{L}=1\left({ }^{56}\right)$; nous imposerons donc cette condition à la cavité. Pour la fréquence qui nous intéresse $\left(\nu=\nu_{a}=3035,7 \mathrm{MHz}, \lambda=9,8755 \mathrm{~cm}\right)$, on trouve alors :

$$
\begin{aligned}
& D=L=13,018 \mathrm{~cm} \\
& V_{\text {cav }}=1732,6 \mathrm{~cm}^{3} \\
& k_{1}=0,58870 \mathrm{~cm}^{-1} \\
& k_{3}=0,24133 \mathrm{~cm}^{-1} \\
& k=0,63624 \mathrm{~cm}^{-1} \\
& Q_{0}=5,4.10^{4} \text { (pour une cavité en cuivre). } \\
& \text { Le cas d'une cavité sphérique a été beaucoup moins étudié ( } 59 \text { ) et il }
\end{aligned}
$$

n'existe. pas de nomenclature standard pour les modes. La carte de l'un de ceux-ci ressemble cependant à celle du mode TE 011 dans une cavité cylindrique et nous lui conser- 

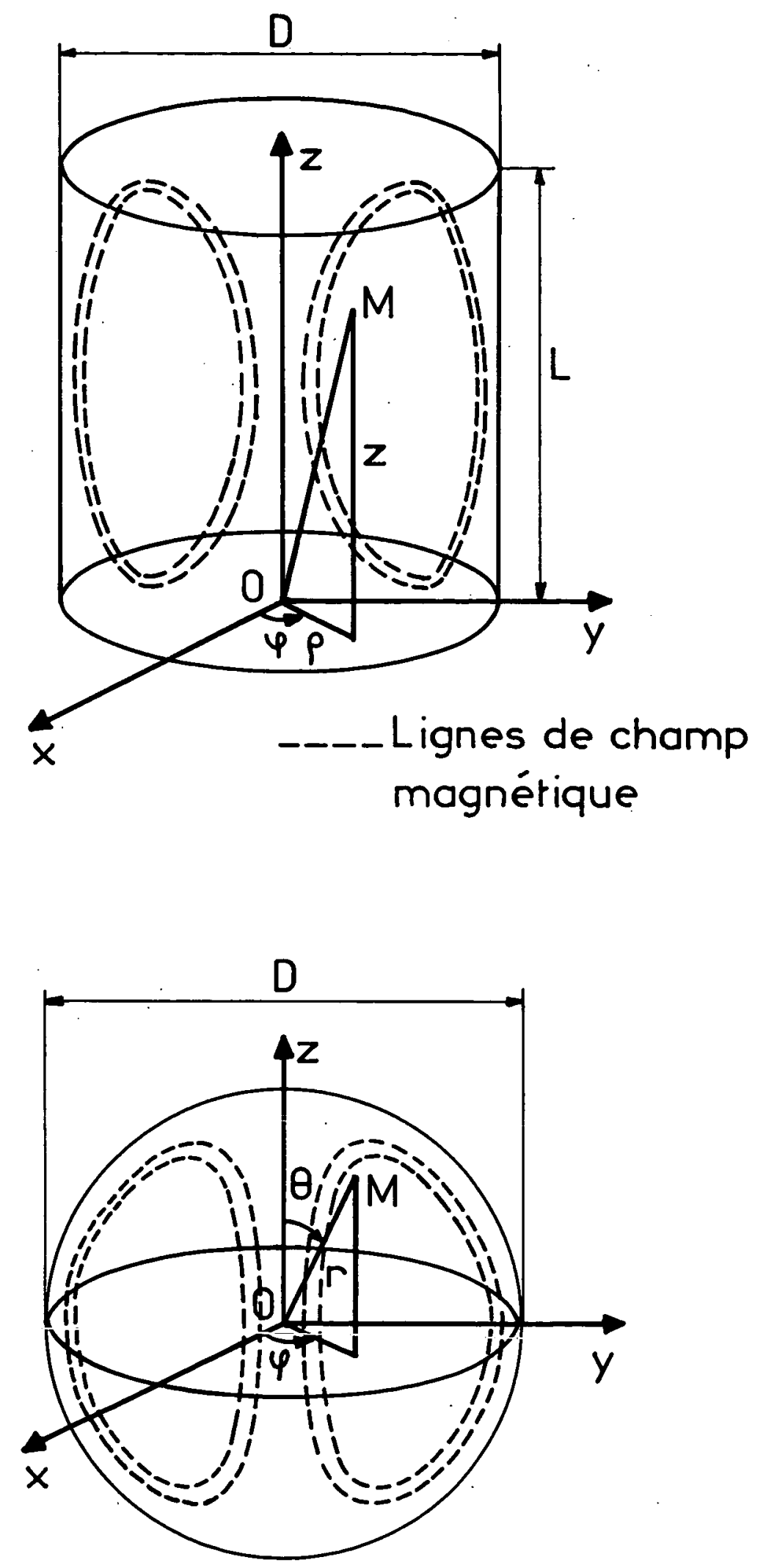

Fig. 29 
verons la même dénominatinn. D désignant le diamètre de la sphère, la fréquence de résonance est donnée par :

$$
\nu=c / \lambda=c k / 2 \pi
$$

avec $k=2 u_{1} / D$, aù $u_{l}=4,4934$ est le premier zéro de la fonction de Bessel $J_{3 / 2}$. Dans un système de coordonnées sphériques d'axe $0 z$ et d’origine 0 située au centre de la sphère, les équations des champs s'écrivent :

$E_{r}=0$

$E_{\theta}=0$

$E_{\phi}=\frac{1}{\sqrt{k r}} J_{3 / 2}(k r) \sin \theta=\sqrt{\frac{2}{\pi}} \frac{1}{k r}\left(\frac{\sin k r}{k r}-\cos k r\right) \sin \theta$

$H_{r}=\frac{2}{(k r)^{3 / 2}} J_{3 / 2}(k r) \cos \theta=2 \sqrt{\frac{2}{\pi}} \frac{1}{(k r)^{2}}\left(\frac{\sin k r}{k r}-\cos k r\right) \cos \theta$

$H_{\theta}=-\frac{1}{(k r)^{3 / 2}}\left[k r J_{1 / 2}(k r)-J_{3 / 2}(k r)\right] \sin \theta=\sqrt{\frac{2}{\pi}} \frac{1}{(k r)^{2}}\left[\sin k r\left(\frac{1}{k r}-k r\right)-\cos k r\right] \sin \theta$

$H_{\phi}=0$

Pour la fréquence qui nous intéresse $(3035,7 \mathrm{MHz})$, on a :

$D=14,125 \mathrm{~cm}$

$v_{\text {cav }}=1476 \mathrm{~cm}^{3}$

$k=0,63624 \mathrm{~cm}^{-1}$

$Q_{0}=5,8 \cdot 10^{4}$ (cavité en cuivre). 


\begin{abstract}
A P P E N I C E I I
MOUVEMENT DES ATOMES ET COEFFICIENT DE REMPLISSAGE

00000000000000000000000000000000000000000000000000
\end{abstract}

Tous les calculs précédents ont été menés dans 1 'hypothèse fondamentale énoncée au chapitre I suivant laquel le les atomes alcalins "voyaient", quel que soit leur mouvement, le champ de radiofréquence appliqué $\vec{H}$ comme un champ homogène de la forme $H_{1 z} \cos \omega t$. Comme nous l'avons vu, ceci entraîne les conséquences suivantes : a) Le signal de détection optique de la résonance 0-0 est de la forme (équation (13) du chapitre II) :

$$
\Delta L_{A} \propto \Delta \sigma_{\mu \mu}^{(2)} \propto \frac{\mu_{B}^{2}\left(\hat{\sigma}_{++}^{(0)}-\hat{\sigma}_{--}^{(0)} H_{1 Z}^{2}\right.}{\left(\frac{1}{T_{2}}\right)^{2}+\left(\omega-\omega_{a}\right)^{2}}
$$

b) La présence du système atomique à l'intérieur d'une cavité résonnante se traduit par l'apparition d'un facteur de surtension atomique $Q_{a}$, donné par (đquations (16) du chapitre IIIJ :

$$
\frac{1}{Q_{a}}=-\frac{4 \pi \mu_{B}^{2}}{K} \frac{n\left(\hat{\sigma}_{++}^{(0)}-\hat{\sigma}_{--}^{(0)}\right)}{\frac{1}{T_{2}}+i\left(\omega-\omega_{a}\right)} \frac{\int_{\operatorname{cell}} H_{1 z} \cdot H_{z} d V}{\int_{c a v} H^{2} d V}=-\frac{K \alpha n \eta}{\delta \omega_{a}+i\left(\omega-\omega_{a}\right)}
$$

Il en résulte en particulier que la résonance (signal de détection optique, ou pic central du signal de détection radioélectrique) a, en l'absence de rétrécissement par réaction, une forme lorentzienne de demi-largeur $\delta \omega_{a}=1 / T_{2}$, où $T_{2}$ représente le temps d'amortissement de la cohérence 0-0. Nous avons vu que l'expérience confirme ce résultat; nous nous proposons dans ce qui suit de justifier théoriquement 
l'hypothèse faite (en montrant en particulier pourquoi aucun élargissement Doppler ne se manifeste dans nos expériences) et de calculer la valeur du coefficient de remplissage $\eta$ dans les cas où nous nous sommes placés.

\section{A) FORME GENERALE DES SIGNAUX DE RESONANCE}

Nous avons appelé $\vec{H}(\vec{r})$ l'amplitude du champ de radiofréquence (onde stationnaire) établi dans la cavité. Les atomes passant au point $\vec{r}$ à l'instant $t$ se trouvent soumis à un champ $H_{z}\left(\vec{r}_{t}\right)\left(e^{i \omega t}+e^{-i \omega t}\right) / 2$ où, par suite de leur mouvement, $\vec{r}_{t}$ doit être considéré comme une fonction du temps. L'équation (5) du chapitre I s'écrit alors :

$$
\frac{d \sigma_{+-}^{(1)}}{d t}=-\left(\frac{1}{T_{2}}+i \omega_{a}\right) \sigma_{+-}^{(1)}-\frac{1}{2} i \mu_{\theta}\left(\hat{\sigma}_{++}^{(0)}-\hat{\sigma}_{--}^{(0)}\right) H_{z}\left(\vec{r}_{t}\right)\left(e^{i \omega t}+e^{-i \omega t}\right)
$$

Nous supposerons, pour effectuer les calculs, que $\left(\hat{\sigma}_{++}^{(0)}-\hat{\sigma}_{--}^{(0)}\right)$ est sensiblement constant d'un point à l'autre de la cellule. L'intégration donne en ce cas, en négligeant le terme non résannant :

$$
\sigma_{+-}^{(1)}=\frac{1}{2} i \mu_{B}\left(\hat{\sigma}_{++}^{(0)}-\hat{\sigma}_{--}^{(0)}\right) e^{-i \omega t} \int_{t_{i}}^{t} e^{-\left[\frac{1}{T_{2}}+i\left(\omega-\omega_{a}\right)\right]\left(t-t^{\prime}\right)} H_{z}\left(\vec{r}_{t},\right) d t^{\prime}
$$

Le moment magnétique correspondant s'écrit :

$$
\begin{aligned}
& M\left(\vec{r}_{t}, t\right)=\frac{\mu_{B}}{h} n\left(\sigma_{+-}+\sigma_{-+}\right)= \\
& \quad=\frac{1}{2} \frac{\mu_{B}^{2}}{h k} n\left(\hat{\sigma}_{++}^{(0)}-\hat{\sigma}_{--}^{(0)}\right)\left[-i e^{i \omega t} \int_{t_{i}}^{t} e^{-\left[\frac{1}{T_{2}}+i\left(\omega-\omega_{a}\right)\right]\left(t-t^{\prime}\right)} H_{z}\left(\vec{r}_{t},\right) d t^{\prime}+c \cdot c \cdot\right]
\end{aligned}
$$

En fait, l'expression ci-dessus n'a tenu compte que des atomes qui se trouvent au point $\vec{r}_{t}$ à l'instant $t$ après s'être trouvés au point $\vec{r}_{t}$, à l'instant t'. 
Pour obtenir la contribution totale des atomes se trouvant au point $\vec{r}$, il faut sommer sur $\vec{r}_{t^{\prime}}=\vec{r}^{\prime}$. Par ailleurs, on peut sans grande erreur considérer que les atomes ont été soumis à la radiofréquence depuis l'instant initial $t_{i}=-\infty$. On a donc finalement : $M(\vec{r}, t)=\frac{1}{2} \frac{\mu_{B}^{2}}{K} \Pi\left[\hat{\sigma}_{++}^{(0)}-\hat{\sigma}_{--}^{(0)}\right] x$

$$
\times\left[-i e^{i \omega t} \int_{-\infty}^{t} d t^{\prime} \int_{c e l l u l e} d^{3} r^{\prime} e^{-\left[\frac{1}{T_{2}}+i\left(\omega-\omega_{a}\right)\right]\left(t-t^{\prime}\right)} H_{z}\left(\vec{r}^{\prime}\right) \mathcal{P}\left(\vec{r}, t, \vec{r}^{\prime}, t^{\prime}\right)+c \cdot c \cdot\right]
$$

où $\mathcal{P}\left(\vec{r}, t, \vec{r}^{\prime}, t^{\prime}\right)$ désigne la probabilité pour que l'atome passe au point $\vec{r}$ à l'instant $t$ après être passé au point $\vec{r}^{\prime}$ à l'instant t'; on a évidemment :

$\int_{\text {cellule }} \mathscr{P}\left(\vec{r}, t, \vec{r}^{\prime}, t^{\prime}\right) d^{3} r=\int_{\text {cellule }} \mathcal{P}\left(\vec{r}, t, \vec{r}^{\prime}, t^{\prime}\right) d^{3} r^{\prime}=1$;

$\mathscr{P}\left(\vec{r}, t, \vec{r}^{\prime}, t\right)=\mathscr{P}\left(\vec{r}, t^{\prime}, \vec{r}^{\prime}, t^{\prime}\right)=\delta\left(\vec{r}-\vec{r}^{\prime}\right)$.

Enfin, nous avons vu au chapitre III que le facteur de surtension atomique $Q_{a}$ était donné par l'expression :

$$
\frac{1}{Q_{a}}=4 \pi i \frac{\int_{\text {cell }} \vec{M}(\vec{r}) \cdot \vec{H} *(\vec{r}) d^{3} r}{\int_{\text {cav }} \vec{H}(\vec{r}) \cdot \vec{H} *(\vec{r}) d^{3} r}
$$

Nous aurons donc ici:

$$
\begin{aligned}
& \frac{1}{Q_{a}}=-\frac{4 \pi \mu_{B}^{2}}{h} n\left(\hat{\sigma}_{++}^{(0)}-\hat{\sigma}_{--}^{(0)}\right) \times \\
& \begin{array}{c}
\frac{1}{\int_{\operatorname{cav}} H^{2}(r) d^{3} r} \int_{-\infty}^{t} d t^{\prime} \int_{c e l l} \int_{c e l l} d^{3} r d^{3} r^{\prime} e^{-\frac{1}{T_{2}}\left(t-t^{0}\right)} H_{z}(\vec{r}) H_{z}\left(\vec{r}^{\prime}\right) P\left(\vec{r}, t, \vec{r}^{\prime}, t^{\prime}\right) x \\
x e^{-i(\omega-\omega)\left(t-t^{\prime}\right)}
\end{array}
\end{aligned}
$$


Nous supposerons que le mouvement aléatoire des atomes est entièrement décrit par la fonction $\mathcal{P}$ et que celle-ci ne dépend que de $t-t^{\prime},=\tau$. En définissant une fonction $g(\tau)$ par les équations :

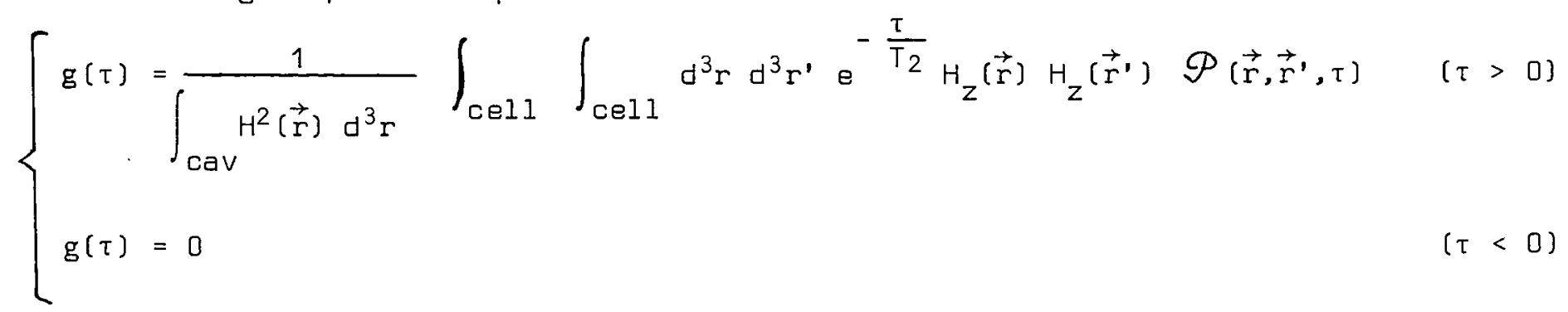

nous avons finalement, avec les notations du chapitre III :

$$
\frac{1}{Q_{a}}=-\operatorname{Kan} \int_{-\infty}^{\infty} g(\tau) e^{-i\left(\omega-\omega_{a}\right) \tau} d \tau=-K \alpha n j\left(\omega-\omega_{a}\right)
$$

Nous obtenons ainsi le résultat suivant lequel $1 / Q_{a}$ est proportionnel à la transformée de Fourier, centrée sur la fréquence $\omega_{a}$, de la fonction de corrélation $g(\tau)$

Par ailleurs, la variation des populations à l'ordre 2 est donnée à l'équilibre par les relations, généralisant celles du chapitre I :

$$
\begin{aligned}
& \frac{d \sigma_{\mu \mu}^{(2)}}{d t}=P_{\mu \mu}^{(2)}+\mathscr{R}_{\mu \mu}^{(2)} \\
& \frac{d \sigma_{++}^{(2)}}{d t}=-\frac{1}{4} \mu_{B}^{2}\left(\hat{\sigma}_{++}^{(0)}-\hat{\sigma}_{--}^{(0)}\right) \times \\
& \times H_{\underline{z}}\left(\vec{r}_{t}\right)\left[\int_{t_{i}}^{t} e^{\left[-\frac{1}{T_{2}}+i\left(\omega-\omega_{a}\right)\right]\left(t-t^{\prime}\right)} H_{z}\left(\vec{r}_{t^{\prime}}\right) d t^{\prime}+c \cdot c \cdot\right]+\mathscr{P}_{++}^{(2)}+\mathcal{R}_{++}^{(2)}=0 \\
& \frac{d \sigma_{--}^{(2)}}{d t}=\frac{1}{4} \mu_{B}^{2}\left(\hat{\sigma}_{++}^{(0)}-\hat{\sigma}_{--}^{(0)}\right) \times \\
& \left.\times H_{z}\left(\vec{r}_{t}\right)\left[\int_{t_{i}}^{t} e^{\left[-\frac{1}{T_{2}}+i\left(\omega-\omega_{a}\right)\right]\left(t-t^{\prime}\right)} H_{z}\left(\vec{r}_{t},\right) d t^{\prime}+c \cdot c \cdot\right]+\mathscr{P}^{(2)}+\mathcal{R}^{(2)}\right]=0
\end{aligned}
$$


Comme nous 1 'avons fait ci-dessus pour $M(\vec{r}, t)$, on peut ici définir

$\Delta \sigma_{\mu \mu}^{(2)}(\vec{r}, t)$; le signal optique sera proportionnel à l'intégrale de cette quantité sur le volume de la cellule. Le calcul est assez long, surtaut à cause des termes d'échange, mais très semblable à celui que nous venons d'effectuer et on aboutit finalement à la formule :

$$
\Delta L_{A} \propto \mu_{B}^{2}\left(\hat{\sigma}_{++}^{(0)}-\hat{\sigma}_{--}^{(0)}\right)\left[\int_{-\infty}^{\infty} g(\tau) e^{-i\left(\omega-\omega_{a}\right) \tau} d \tau+c . c \cdot\right]
$$

dans laquelle intervient la même fonction de corrélation $g(\tau)$ que précédemment. La résonance a donc, dans les deux cas, la même forme, dont le calcul se ramène à celui de la fonction $g(\tau)$. Quelle que soit celle-ci, l'aire de la résonance est d'ailleurs toujours la même : on a en effet :

$$
\begin{array}{r}
\int_{-\infty}^{\infty} j\left(\omega-\omega_{a}\right) d \omega=\pi g(0)=\frac{\pi}{\int_{\operatorname{cav}} H^{2}(\vec{r}) d^{3} r} \int_{c e l l} \int_{c e l l} d^{3} r d^{3} r^{\prime} H_{z}(\vec{r}) H_{z}\left(\vec{r}^{\prime}\right) \delta\left(\vec{r}-\vec{r}^{\prime}\right)= \\
=\frac{\pi<H_{z}^{2}(\vec{r})>_{\operatorname{cell}} V_{\operatorname{cell}}}{<H^{2}(\vec{r})>_{\text {cav }} V_{\text {cav }}}
\end{array}
$$

Cette aire est donc proportionnelle à la valeur moyenne, prise sur la cellule, du carré de la composante du champ de radiofréquence suivant la direction du champ magnétique statique.

Nous allons calculer $g(\tau)$ dans les cas où nous nous sommes placés.

\section{B) CELLULE SANS GAZ ETRANGER}

Lorsque $\left(t-t^{\prime}\right)=\tau+\infty, l^{\prime}$ atome parti de $\vec{r}^{\prime}$ à l'instant $t$ a une probabilité égale de se trouver en tout point de la cellule et donc $\mathscr{P}(\vec{r}, \vec{r},, \tau)$ tend vers $1 / V_{\text {cell }}$. En posant $\mathscr{P}\left(\vec{r}, \vec{r}^{\prime}, \tau\right)=1 / V_{\text {cell }}+\left[\mathcal{P}\left(\vec{r}, \vec{r}^{\prime}, \tau\right)-1 / V_{\text {cell }}\right)$, nous avons : 


$$
\begin{aligned}
& g(\tau)=\frac{e^{-\frac{\tau}{T_{2}}}}{V_{c e l l} \int_{c a v} H^{2}(\vec{r}) d^{3} r} \int_{c e l l} \int_{c e l l} d^{3} r d^{3} r^{\prime} H_{z}(\vec{r}) H_{z}\left(\vec{r}^{\prime}\right) \\
& \begin{aligned}
+\frac{1}{\int_{\text {cav }} H^{2}(\vec{r}) d^{3} r} \int_{c e l l} \int_{c e l l} d^{3} r d^{3} r^{\prime} H_{z}(\vec{r}) H_{z}\left(\vec{r}^{\prime}\right) e^{-\frac{\tau}{T_{2}}} \times \\
x\left(P\left(\vec{r}, \vec{r}^{\prime}, \tau\right)-1 / V_{c e l l}\right)
\end{aligned}
\end{aligned}
$$

Le premier terme peut s'écrire sous la forme $e^{-\frac{T}{T_{2}}}<H_{z}(\vec{r})>_{c e l l}^{2} V_{c e l l} l^{\prime}$ $<H^{2}(\vec{r})>_{\text {cav }} V_{\text {cav; }}$ la transformation de fourier lui fait correspondre une raie de largeur $\delta \omega_{a}=1 / T_{2}$. Le second par contre apparait difficile à calculer. Nous savons cependant qu'il décroît fortement lorsque la répartition des atomes dans la cellule de diamètre $D$ tend à devenir uniforme, c'est-à-dire au bout d'un temps $\tau$ comparable au temps de vol $\tau_{v}=D / \bar{v}$ des atomes d'une paroi à l'autre et donc très inférieur à $T_{2}$. En supposant quelque peu arbitrairement pour la raie correspondante une forme lorentzienne de demi-largeur $1 / \tau_{v}$, le signal de résonance peut donc s'écrire, compte tenu de la valeur de l'aire totale calculée plus haut :

$$
\begin{aligned}
& \operatorname{Re} j\left(\omega-\omega_{a}\right)=\frac{\left\langle H_{z}(\vec{r})\right\rangle_{\text {cell }}^{2} V_{\text {cell }}}{\left\langle H^{2}(\vec{r})\right\rangle_{\text {cav }} V_{\text {cav }}} \frac{1 / T_{2}}{\left(\frac{1}{T_{2}}\right)^{2}+\left(\omega-\omega_{a}\right)^{2}}+ \\
& +\frac{\left[<H_{z}^{2}(\vec{r})>_{\operatorname{cell}}-<H_{z}(\vec{r})>_{\text {cell }}^{2}\right] V_{\text {cell }}}{<H^{2}(\vec{r})>_{\operatorname{cav}} v_{\operatorname{cav}}} \frac{1 / \tau_{v}}{\left\{\frac{1}{\tau_{v}}\right)^{2}+\left(\dot{w} \dot{w}_{a}\right)^{2}}
\end{aligned}
$$

La largeur $1 / \tau_{v}=\bar{v} / D$ est de l'ordre de la largeur Doppler $\bar{v} / \lambda$; on retrouve ainsi un résultat déjà énoncé dans le cas du maser à hydrogène (22). Dans la bande de fréquences intéressante, $\left|\omega-\omega_{a}\right| \hat{c} \delta \omega_{a}$, le second terme est négligeable 
par rapport au premier tant que $\left\langle H_{z}(\vec{r})>_{\text {cell }}^{2}>\frac{\tau_{V}}{T_{2}}\left[<H_{z}^{2}(\vec{r})>_{\text {cell }}-<H_{z}(\vec{r})>_{\text {cell }}^{2}\right]\right.$, condition sur laquelle nous reviendrons plus loin. Tout se passe donc bien alors comme si les atomes étaient soumis à un champ moyen $H_{1 z}=\left\langle H_{z}(\vec{r})\right\rangle_{\text {cell }}$; aucun élargissement Doppler n'apparait, la résonance a une demi-largeur $\delta \omega_{a}=1 / T_{2}$ et le coefficient de remplissage est donné par la relation :

$$
\eta=\frac{\left\langle H_{z}(\vec{r})\right\rangle_{\text {cell }}^{2} V_{\text {cell }}}{\left\langle H^{2}(\vec{r})\right\rangle_{\text {cav }} V_{\text {cav }}}
$$

Nous allons calculer $n$ pour une cavité cylindrique de diamètre $D$ et de longueur $L$, contenant une cellule cylindrique de diamètre $D_{i}$ et de longueur $L_{i}$ et résonnant sur le mode TE 011. D'après les équations du champ données à l'appendice I, nous avons, en continuant à supposer l'axe dela cavité parallèle au champ statique : $<H^{2}(\vec{r})>_{\operatorname{cav}}=\frac{8}{k^{2} D^{2} L} \int_{0}^{D / 2} \rho d \rho \int_{0}^{L} d z\left[k_{3}^{2} J_{0}^{\prime 2}\left(k_{1} \rho\right) \cos ^{2} k_{3} z+k_{1}^{2} J_{0}^{2}\left(k_{1} \rho\right) \sin ^{2} k_{3} z\right]$ Compte tenu des relations (60) :

$$
\begin{aligned}
J_{0}^{\prime 2}\left(k_{1} \rho\right) & =J_{1}^{2}\left(k_{1} \rho\right) \\
\int J_{0}^{2}\left(k_{1} \rho\right) \rho d \rho & =\frac{1}{2} \rho^{2}\left[J_{1}^{2}\left(k_{1} \rho\right)+J_{0}^{2}\left(k_{1} \rho\right)\right] \\
\int J_{1}^{2}\left(k_{1} \rho\right) \rho d \rho & =\frac{1}{2} \rho^{2}\left[J_{1}^{\prime 2}\left(k_{1} \rho\right)+\left(1-\frac{1}{k_{1}^{2} \rho^{2}} J_{1}^{2}\left(k_{1} \rho\right)\right]\right. \\
J_{1}^{\prime 2}\left(k_{1} \rho\right) & =\frac{1}{4}\left[J_{0}\left(k_{1} \rho\right)-J_{2}\left(k_{1} \rho\right)\right]^{\frac{1}{2}}
\end{aligned}
$$

il vient :

$$
\left.\begin{array}{rl}
<H^{2}(\vec{r})>_{\text {Cav }}= & \left\{\frac { 2 \rho ^ { 2 } } { k ^ { 2 } k _ { 3 } D ^ { 2 } L } \left[k_{3}^{2}\left[k_{3} z+\frac{1}{2} \sin 2 k_{3} z\right]\left[\frac{J_{0}^{2}}{4}+\frac{J_{2}^{2}}{4}-\frac{J_{0} J_{2}}{2}+\left(1-\frac{1}{k_{1}^{2} \rho^{2}}\right) J_{1}^{2}\right]\right.\right. \\
& \left.+k_{1}^{2}\left[k_{3} z-\frac{1}{2} \sin 2 k_{3} z\right]\left[J_{0}^{2}+J_{1}^{2}\right]\right]
\end{array}\right\} \begin{aligned}
& \rho=D / 2 \quad z=L \\
& \rho=0 \quad z=0
\end{aligned}
$$


soit, tous calculs faits :

$$
<H^{2}(\vec{r})>_{\text {cav }}=0,0811
$$

D'autre part :

$$
\begin{aligned}
& \left\langle H_{z}(\vec{r})>_{\operatorname{cell}}=\frac{8 k_{1}}{k D_{i}{ }^{2} L_{i}} \int_{0}^{D_{i} / 2} \rho d \rho \int_{L / 2-L_{i} / 2}^{L / 2+L_{i} / 2} d z J_{0}\left(k_{1} \rho\right) \sin k_{3} z\right. \\
& \left.=-\frac{8}{k k_{3} D_{i}^{2} L_{i}}\left\{\rho J_{I}(k) \rho\right)\right\}\left\{\begin{array} { l } 
{ \rho = D _ { i } / 2 } \\
{ \rho = 0 }
\end{array} \quad \left\{\begin{array}{l}
\cos k_{3} z \\
z=L / 2-L_{i} / 2
\end{array}\right.\right.
\end{aligned}
$$

D'où :

$\left\langle H_{z}(\vec{r})>_{\text {cell }}^{2}=\frac{64}{k^{2} \pi^{2} D_{i}{ }^{2}\left(\frac{L_{i}}{L}\right)^{2}} J_{l}^{2}\left(u_{01}^{\prime} \frac{D_{i}}{D}\right) \sin ^{2}\left(\frac{\pi}{2} \frac{L_{i}}{L}\right)\right.$

et enfin :

$$
n=79,95 \frac{J_{1}{ }^{2}\left(u_{01}^{\prime} \frac{D_{i}}{D}\right) \sin ^{2}\left(\frac{\pi}{2} \frac{L_{i}}{L}\right)}{k^{2} D^{2}\left(\frac{L_{i}}{L}\right)}
$$

Le coefficient de remplissage passe par un maximum égal à 0,45 pour $D_{i} / D=0,48$ et $L_{i} / L=0,74$. Notons que la valeur du diamètre $D_{i}$ ainsi trouvée est assez malencontreuse, cer. elle conduit à une cellule dont les parois se trouvent dans une région de champ électrique maximum : cette valeur de $n$ correspondra donc pratiquement à une iñuvivaise surtension, ce qui en réduit l'intérêt. Nul pour $D_{i}=0$, le coefficient de remplissage donné par l'expression ci-dessus s'annule également si $D_{i}=0$, c'est-à-dire en particulier pour une cellule occupant toute la cavité. Le premier terme de $R e j\left(\omega-\omega_{a}\right)$ disparait alors; seul subsiste le second, de largeur $1 / \tau_{v}$ beaucoup plus grande et dont 1 'amplitude au sommet est faible. 
En accord avec ces résultats, nous avons obtenu un signal d'amplification relativement important $(p \simeq 0,4)$ avec des cellules sphériques de diamètre $100 \mathrm{~mm}$ (configuration E de la figure 12), assez équivalentesà la cellule cylindrique correspondant au coefficient de remplissage maximum: une cellule cylindrique de configuration c occupant toute la cavité n'a par contre donné lieu à aucun signal observable.

\section{C) CELLULE AVEC GAZ ETRANGER}

Il faut cette fois connaitre la variation de $\mathscr{P}\left(\vec{r}, \vec{r}^{\prime}, \tau\right)$ en présence de gaz. Un calcul analogue a été effectué par DICKE (6l) dans le cas d'atomes soumis à une onde progressive et diffusant dans un milieu infini; il conduit au résultat désormais classique suivant lequel le terme d'élargissement lié au mouvement a une forme lorentzienne dont la demi-largeur $\delta w$ est reliée à la demi-largeur Doppler par la relation $\delta \omega=$ (2.8 $\left.\ell_{t} / \lambda\right) \delta \omega_{\text {Doppler' }}{ }_{t}$ étant le libre parcours moyen de transport relatif à l'atome étudié et $\lambda$ la longueur d'onde de la transition considérée. En introduisant le coefficient de diffusion D comme nous l'avons fait au chapitre VI, on s'assure aisément que $\delta w$ n'est autre que l'inverse du temps nécessaire à l'atome pour diffuser sur une distance moyenne égale à $\lambda / 4$. Pour la longueur d'onde $(10 \mathrm{~cm}$ ) et les pressions (quelques torrs) qui nous intéressent, cet élargissement est négligeable par rapport à la demi-largeur "naturelle" $\delta \omega_{a}=1 / T_{2}$; en ce qui concerne l'action de la radiofréquence, tout se passe comme si l'atome était pratiquement immobile et on a alors la relation très simple :

$$
\mathscr{P}\left(\vec{r}, \vec{r}^{\prime}, t\right)=\delta\left(\vec{r}-\vec{r}^{\prime}\right)
$$

soit encore :

$$
g(\tau)=\frac{e^{-\tau / T_{2}}}{\int_{\operatorname{cav}} H^{2}(\vec{r}) d^{3} r} \int_{c e l l} \int_{\text {cell }} d^{3} r d^{3} r^{\prime} H_{z}(\vec{r}) H_{z}\left(\vec{r}^{\prime}\right) \delta\left(\vec{r}-\vec{r}^{\prime}\right)
$$


La résonance a donc la forme :

$$
\operatorname{Re} j\left(\omega-\omega_{a}\right)=\frac{\left\langle H_{z}{ }^{2}(\vec{r})\right\rangle_{\operatorname{cell}} V_{\operatorname{cell}}}{\left\langle H^{2}(\vec{r})\right\rangle_{\operatorname{cav}} V_{\operatorname{cav}}} \frac{1 / T_{2}}{\left(\frac{1}{T_{2}}\right)^{2}+\left(\omega-\omega_{a}\right)^{2}}
$$

Sa demi-largeur $\delta \omega_{a}$ est ici encore égale à $1 / T_{2}$. Les relations établies dans l'hypothèse fondamentale du chapitre I sont donc encore valables, mais le coefficient de remplissage est cette fois donné par l'équation :

$$
n=\frac{\left\langle H_{z}^{2}(\vec{r})\right\rangle_{\operatorname{cell}} V_{\text {cell }}}{\left\langle H^{2}(\vec{r})\right\rangle_{\text {cav }} V_{\text {cav }}}
$$

Il est plus grand dans les mêmes conditions qu'en l'absence de gaz, puisque $<H_{z}^{2}(\vec{r})>$ est toujours supérieur à $\left\langle H_{z}(\vec{r})>^{2}\right.$. Ce résultat doit toutefois être accueilli avec prudence : en présence de gaz, l'hypothèse d'une répartition uniforme des atomes en état d'inversion de populations n'est souvent plus justifiée, comme nous l'avons vu au chapitre VI et le coefficient de remplissage réel peut différer considérablement de la valeur calculée d'après la formule ci-dessus.

Nous allons évaluer $\eta$ pour la cellule de diamètre $D_{i}$ et de longueur $L_{i}$ déjà considérée au paragraphe précédent. Nous avons ici :

$$
\begin{aligned}
& <H_{z}{ }^{2}(\vec{r})>_{\operatorname{cell}}=\frac{8 k_{1}{ }^{2}}{k^{2} D_{i}{ }^{2} L_{i}} \int_{0}^{D_{i} / 2} \rho d \rho \int_{L / 2-L_{i} / 2}^{L / 2+L_{i} / 2} d z J_{0}{ }^{2}\left(k_{1} \rho\right) \sin ^{2} k_{3} z
\end{aligned}
$$

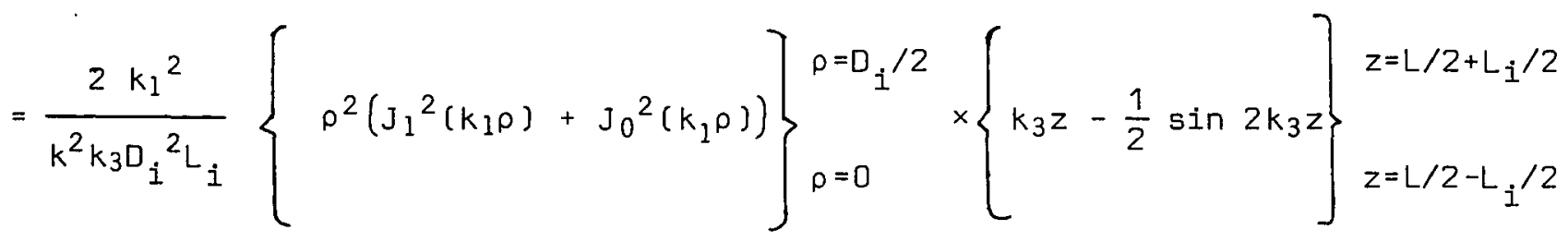


D'où finalement :

$$
n=115,24 \frac{\left(\frac{D_{i}}{D}\right)^{2}\left[J_{0}^{2}\left(u_{01}^{\prime} \frac{D_{i}}{D}\right)+J_{1}^{2}\left(u_{01}^{\prime} \frac{D_{i}}{D}\right)\right]\left[\pi \frac{L_{i}}{L}+\sin \left(\pi \frac{L_{i}}{L}\right)\right]}{k^{2} D^{2}}
$$

Le coefficient de remplissage est, cette fois, une fonction croissante des dimensions de la cellule et atteint la valeur $n=0,856$ lorsque celle-ci occupe toute la cavité. Nous avons effectivement vérifié que la cellule cylindrique de configuration c dont nous avons parlé au paragraphe précédent, pour laquelle aucun signal n’était observable en l'absence de gaz, donnait lieu à une amplification importante lorsqu'on y introduisait ultérieurement le gaz étranger suivant la technique décrite au chapitre $V$; après optimisation, nous avons actuellement obtenu pour cette cavité une valeur du paramètre d'amplification $p=0,86$.

Nous avons également calculé $\eta$ pour une cellule sphérique de diamètre $D_{i}$ avec gaz étranger, contenue dans une cavité sphérique de diamètre D résonnant sur le mode TE 011. Nous avons alors :

$$
\begin{aligned}
<H^{2}(\vec{r})>_{\text {Cav }} & =\frac{12}{k^{3} D^{3}} \int_{0}^{D / 2} r^{2} d r \int_{0}^{\pi} \sin \theta d \theta \frac{1}{\Gamma^{3}} \times \\
& \times\left[4 J_{3 / 2}^{2}(k r) \cos ^{2} \theta+\left(k r J_{1 / 2}(k r)-J_{3 / 2}(k r)\right)^{2} \sin ^{2} \theta\right]
\end{aligned}
$$

$\left\langle H_{z}^{2}(\vec{r})>_{\operatorname{cel} I}=\frac{12}{k^{3} D_{i}^{3}} \int_{0}^{D_{i} / 2} r^{2} d r \int_{0}^{\pi} \sin \theta d \theta \frac{1}{r^{3}} \times\right.$

$$
\begin{array}{r}
4 \mathrm{~J}_{3 / 2}^{2} \cos ^{4} \theta+\left(k r \mathrm{~J}_{1 / 2}-\mathrm{J}_{3 / 2}\right)^{2} \sin ^{4} \theta+4 \mathrm{~J}_{3 / 2}\left(\mathrm{kr} \mathrm{J}_{1 / 2}-\mathrm{J}_{3 / 2}\right) \\
\left.\sin ^{2} \theta \cos ^{2} \theta\right]
\end{array}
$$


Après quelques manipulations, on obtient :
$\left.=\frac{\left\{\frac{4(\cos 2 x-1)}{x^{3}}+\frac{8 \sin 2 x}{x^{2}}-\frac{4(\cos 2 x+1)}{x}-4 \sin 2 x+8 x\right\}_{x=k D_{i} / 2}}{\left\{\frac{10(\cos 2 x-1)}{x^{3}}+\frac{20 \sin 2 x}{x^{2}}-\frac{10(\cos 2 x+1)}{x}-5 \sin 2 x+10 x\right\}}\right\}_{x=k D / 2}$

En particulier, pour une cellule remplissant toute la cavité $\left(D_{i}=\mathrm{D}\right)$, on trouve $n=0,798$. 


\section{TECHNOLOGIE DE CONSTRUCTION DE L' ENSEMBLE CELLULE-.CAVITE 000000000000000000000000000000000000000000000000000000000 .}

Nous nous proposons, dans se qui suit, de revenir plus en détail sur les difficultés technologiques rencontrées lors de la mise au point d’un ensemble cellulecavité répondant aux impératifs nécessaires, dont nous avons donné la liste au chapitre V. Rappelons que nous avons été amenés à étudier six configurations différentes (fig. 12). Quatre d'entre elles font effectivement appel à une cellule pour assurer l'étanchéité :

Configuration C : cellule cylindrique mince dans une cavité cylindrique étanche assurant un prévide;

D : la même, avec deux fenêtres pour le pompage optique;

E : cellule sphérique mince dans une cavité cylindrique ouverte à l'atmosphère;

F : cellule sphérique mince dans une cavité sphérique ouverte.

Dans les deux dernières configurations, une cavité étanche contient directement le rubidium sans cellule intermédiaire :
A : cavité cylindrique en cuivre;
B : cavité cylindrique en acier inoxydable.
Nous allons examiner successivement ces différents cas. 


\section{A) CONFIGURATIONS AVEC CELLULE INTERIEURE}

Le gros problème est dans ce cas d'obtenir la cellule elle-même. Les considérations de faibles pertes diélectriques et de transparence amènent à choisir la silice fondue comme matériau de base. Moyennant des délais de livrais'on assez longs ( 4 mois), nous n'avons pas eu trop de difficultés à obtenir de l'industrie (62) la réalisation de cellules sphériques soufflées, d'un diamètre compris entre 100 et $135 \mathrm{~mm}$ et dont l'épaisseur évaluée par pesée est de l'ordre de 1,5 mm. Reliées au banc de pompage, lors du remplissage, par leur queusot suivi d'un raccord quartzpyrex, elles supportent parfaitement la mise sous vide et l'étuvage.'Leur mise en place dans la cavité entraine d'importantes modifications des dimensions de celle-ci pour retrouver l'accord. La longueur intérieure d'une cavité cylindrique de diamètre intérieur $132 \mathrm{~mm}$ passe ainsi, à l'accord, de 121 à $100 \mathrm{~mm}$ lors de l'intraduction d'une cellule de diamètre $100 \mathrm{~mm}$, qui est par suite la plus grande que l'on puisse employer dans ces conditions. Dans le cas d'une cavité sphérique, il a fallu opérer par tâtonnements pour aboutir à un ensemble cellule-cavité résonnant à la bonne fréquence et tel que la paroi de la cellule soit très proche de la surface interne de la cavité; nous avons obtenu une solution satisfaisante avec une cellule de $135 \mathrm{~mm}$ dans une cavité de $139 \mathrm{~mm}$. La surtension de la cavité vide est alors beaucoup moins perturbée que dans le cas précédent et s'abaisse seulement de 50000 à 40000 (contre, respectivement, 45000 et 18000 dans le cas de la cavité cyíindriquej.

L'obtention d'une cellule cylindrique est par contre plus difficile. Des essais préliminaires nous ont montré qu'une cellule à parois de $6 \mathrm{~mm}$ d'épaisseur, même en silice fondue, perturbait gravement la surtension de la cavité; or cette épaisseur 
de $6 \mathrm{~mm}$ est considérée comme nécessaire par les fabricants pour que les faces planes d'un diamètre de $130 \mathrm{~mm}$ puissent résister à la pression atmosphérique (effort $130 \mathrm{~kg}$ ) Nous avons ainsi été amenés à envisager des cellules plus minces (épaisseur $2,5 \mathrm{~mm}$ ) protégées par un prévide extérieur. La réalisation de ces cellules s'est révélée beaucoup plus difficile que celle des cellules sphériques et les délais de livraison s'en sont allongés d’autant (un an). Le point le plus délicat est la soudure des fonds sur le corps cylindrique. L'assemblage par poli optique n'a pu être employé par suite de la minceur des parois; par ailleurs, la nécessité d'éviter les déformations lors d'une soudure par fusion conduit à effectuer celle-ci à la température la plus basse possible, ce qui laisse souvent subsister des fuites dont la recherche est rendue difficile par l'impossibilité d'évacuer la cellule sans précautions.

La construction des cavités relatives à ces configurations n'a par contre pas posé de problèmes particuliers. En effet, nous mettions simultanément au point les cavités étanches relatives aux configurations A et $B$ dont nous parlerons plus loin. Dans le cas de la configuration E (cellule sphérique dans une cavité cylindrique), il nous a suffi d'employer une des cavités précédentes sans joint d'étanchéité, un fond réentrant permettant de raccourcir la cavité et de rattraper ainsi l'effet de la cellule sur l'accord. Dans le cas de la configuration C cellule cylindrique mince de diamètre $130 \mathrm{~mm}$ et de longueur $110 \mathrm{~mm}$, placée dans une cavité cylindrique de diamètre $132 \mathrm{~mm}$ ), le raccourcissement nécessaire est faible $(7 \mathrm{~mm})$ et il a suffi de réusiner légèrement l'un des fonds pour l'obtenir; par ailleurs, les exigences relatives au prévide étant moins sévères, les solutions imparfaitement mises au point pour la configuration $A$ ont pu convenir. L'assemblage final de la double enceinte à vide lle raccord comprend une dizaine de verres différents pour réduire les efforts dus à la dilatation) est par contre 
acrobatique. La cavité de configuration $F$ a pu être obtenue facilement par tournage sphérique. Dans ce dernier cas, comme d'ailleurs dans la configuration E, les boucles de couplage peuvent être facilement changées, ce qui est évidemment très intéressant.

\section{B) CONFIGURATIONS SANS CELLULE INTERIEURE}

Les premiers essais ayant montré la difficulté de réaliser une cellule ne perturbant que peu la surtension de la cavité, nous avons été amenés à envisager une cavité étanche contenant le rubidium sans cellule intermédiaire. En plus des qualités qui lui étaient déjà demandées précédemment, la cavité doit alors être étuvable à $350^{\circ} \mathrm{C}$ au minimum, étanche en vide permanent et "paraffinable" par la méthode habituelle. Les problèmes technologiques ainsi posés nous ont paru dès l'abord très difficiles, impression qui n'a cessé de se renforcer par la suite au cours de nombreux essais infructueux. Nous pensons utile de décrire toutes les solutions que nous avons étudiées, y compris celles dont l'échec pouvait sembler prévisible aux spécialistes : nous rendrons ainsi au moins disponible aux chercheurs intéressés une documentation dont une grande partie ne figure pas dans la littérature technique ou est d'un accès malaisé.

Compte tenu des exigences imposées, le cuivre nous a semblé le matériau de départ le mieux adapté. Le premier problème qui se pose est alors d'assujettir, sur les fonds d'une cavité construite en ce métal, des fenêtres permettant le passage de la luimì̀re de pompage.

(a) La solution la plus courante en vide entretenu et qui permet dans ce cas d'atteindre des pressions très basses $\left(<10^{-7}\right.$ torr $)$ consiste à serrer les fenêtres contre le métal avec interposition d'un joint en élastomère logé dans une gorge de forme adaptée. Nous avons essayé des joints toriques en silicone, ce matériau devant en principe permettre un étuvage à température modérée $\left(250^{\circ}\right)$. L'expérience nous a montré que le silicone 
perdait toute propriété élastique à cette température et présentait de plus une porosité excessive ( $\left.{ }^{63}\right)$. L'emploi de joints en viton ou en polyimide (64) aurait réduit ces inconvénients mais n'aurait pas non plus permis de maintenir un vide non entretenu. (b) On peut envisager de coller les fenêtres sur le métal au moyen d’une résine à basse tension de vapeur (époxy, silicone, polyimide) ( ${ }^{65}$ ). Bien que pour certains emplois, la méthode donne satisfaction même en vide statique (66), nos essais nous ont montré 1 'insuffisance de cette solution surtout en présence d"un élément aussi réactif que le rubidium. Le collage au chlorure d'argent $\left({ }^{67}\right.$ ) aurait peut-être été préférable mais nous a semblé trop fragile.

(C) Nous avons alors pensé à serrer les fenêtres sur les fonds en cuivre au moyen d'un métal plastique : les exigences d'étuvage orientent vers le choix de l'or. Une solution de ce type (avec joints d'aluminium) avait déjà été proposée dans la littérature (68). Nos essais ont été menés avec une:fenêtre en Pyrex de $60 \mathrm{~mm}$ de diamètre et $4 \mathrm{~mm}$ d'épaisseur, obturant un trou circulaỉe de $46 \mathrm{~mm}$ de diamètre, le joint d'or interposé ayant la forme d'un tore de $49 \mathrm{~mm}$ de diamètre intérieur et $2 \mathrm{~mm}$ d'épaisseur. Nous avons obtenu une bonne étanchéité à froid, moyennant une force de serrage considérable (de 1'ordre de $100 \mathrm{~kg}$ par centimètre de joint] qui nécessitait d'ailleurs des pièces de cuivre très épaisses; malheureusement, la fenêtre se cassait régulièrement à l'étuvage, lorsqu'on atteignait une température de $150^{\circ} \mathrm{C}$ environ. Dans l'espoir de réduire la force de serrage nécessaire, nous avons essayé de déposer par voie chimique une couche d’or sur le verre au niveau du joint : les résultats n'en ont pas été améliorés et nous avons dû abandonner cette solution.

(d) Nous ne pouvions pas employer la solution la plus robuste et la plus classique pour réaliser un raccord verre-métal, qui consiste à souder a!j verre un métal ou alliage à 
dilatation adaptée; ces derniers sont en effet tous ferromagnétiques, à l'exception du molybdène avec lequel des scellements tubulaires de grand diamètre ne semblent guère possibles. Nous avons cependant été contraints, pour des questions de robustesse. d'utiliser des scellements Kovar-verre moly pour les queusots latéraux, soumis à des efforts lors du scellement final, ainsi que pour la boucle de couplage; mais la perturbation magnétique, à la rigueur tolérable pour de petites pièces éloignées de I'axe de la cavité, aurait été inadmissible pour des fenêtres.

On sait que le verre, de faible résistance mécanique surtout à la traction, ne peut en principe être soudé à un métal que si le coefficient de dilatation de ce dernier diffère de moins de $10 \%$ de celui du verre employé lla même candition est valable pour la soudure entre eux de deux verres différents). La combinaison Kavarverre moly (dilatation $50.10^{-7} /{ }^{\circ} \mathrm{C}$ ) répond en particulier à cette condition. Aucun verre ne possède un coefficient de dilatation s'approchant de celui du cuivre $\left(170.10^{-7} /{ }^{\circ} \mathrm{C}\right)$. La soudure verre-cuivre est cependant possible grâce. à un artifice appelé "scellement Housekeeper" $\left({ }^{69}\right)$. Celui-ci consiste à amincir le clivre, au niveau de la soudure, jusqu'à une épaisseur très faible $(0,15 \mathrm{~mm})$ : le cuivre suit alors plastiquement les déformations liées aux dilatations, sans entraîner dans le verre des efforts amenant la rupture de celui-ci. Cette technique, employée avant la guerre jusqu'à de très grandes dimensions (scellements tubulaires de $150 \mathrm{~mm}$ de diamètre), a presque totalement disparu depuis, avec l'apparition des scellements Kovar-moly de réalisation beaucoup plus facile et dont la robustesse est très supérieure; nous avons été conduits à demander à l'industrie sa remise en usage. Nous avons d'abord eu des problèmes avec la qualité du cuivre. La nuance OFHC est indispensable, au niveau des scellements pour des questions d'adhérence et de non porosité, mais aussi pour le corps de la 
cavité qui doit subir, comme nous le verrons, des brasures sous hydrogène : le passage au four à hydrogène fissure en effet irrémédiablement le cuivre électrolytique. Le matériau une fois obtenu, nous avons constaté que les scellements, malgré une protection extérieure à l'aquadag, se mettaient fréquemment à fuir durant l'étuvage; si certaines réalisations ont bien tenu, nous $n^{\circ}$ avons pas encore réussi à mettre au point une solution sure. Il est, de plus, possible que ces scellements, où l'adhérence entre le cuivre et le verre est assurée par l'intermédiaire d'une pellicule d’axyde cuivreux, soient attaqués à la longue par la vapeur de rubidium.

(e) Il faut ensuite, une fois en possession de fonds porteurs d'une fenêtre, trouver une méthode pour réaliser l'étanchéité corps de cavité-fond sans détruire l'étanchéité fondfenêtre. Nous avons essayé de l'obtenir par un joint torique en or de $150 \mathrm{~mm}$ de diamètre et 1 ou $2 \mathrm{~mm}$ d’épaisseur, serré entre deux brides planes ( 70 ). Les forces de serrage à exercer sont considérables (de l'ordre de $300 \mathrm{~kg}$ par centimètre de joint, soit $14000 \mathrm{~kg}$ sur toute la périphérie) et difficiles à maintenir avec des brides de cuivre qui, bien que renforcées de contre-brides en acier inoxydable, avaient tendance à fluer même sous forte épaisseur. Nous n'avons jamais réussi à obtenir une étanchéité totale à froid; à plusieurs reprises cependant, l'étanchéité est devenue excellente après étuvage. Nous avons attribué ce phénomène à la diffusion de l'or dans le cuivre, conduisant à une soudure "à froid" ( $\left.{ }^{71}\right)$; effectivement, leṣ fonds deviennent alors inséparables du corps de la cavité. Une petite cavité d'essai ainsi construite ne montre actuellement aucune trace de fuite, après quatre années de stockage. Malheureusement, nous n’avons ici pas réussi à rendre la solution sûre : il subsiste souvent des fuites, peut-être attribuables, dans certains cas, à la formation d'un pseudo-eutectique poreux. 


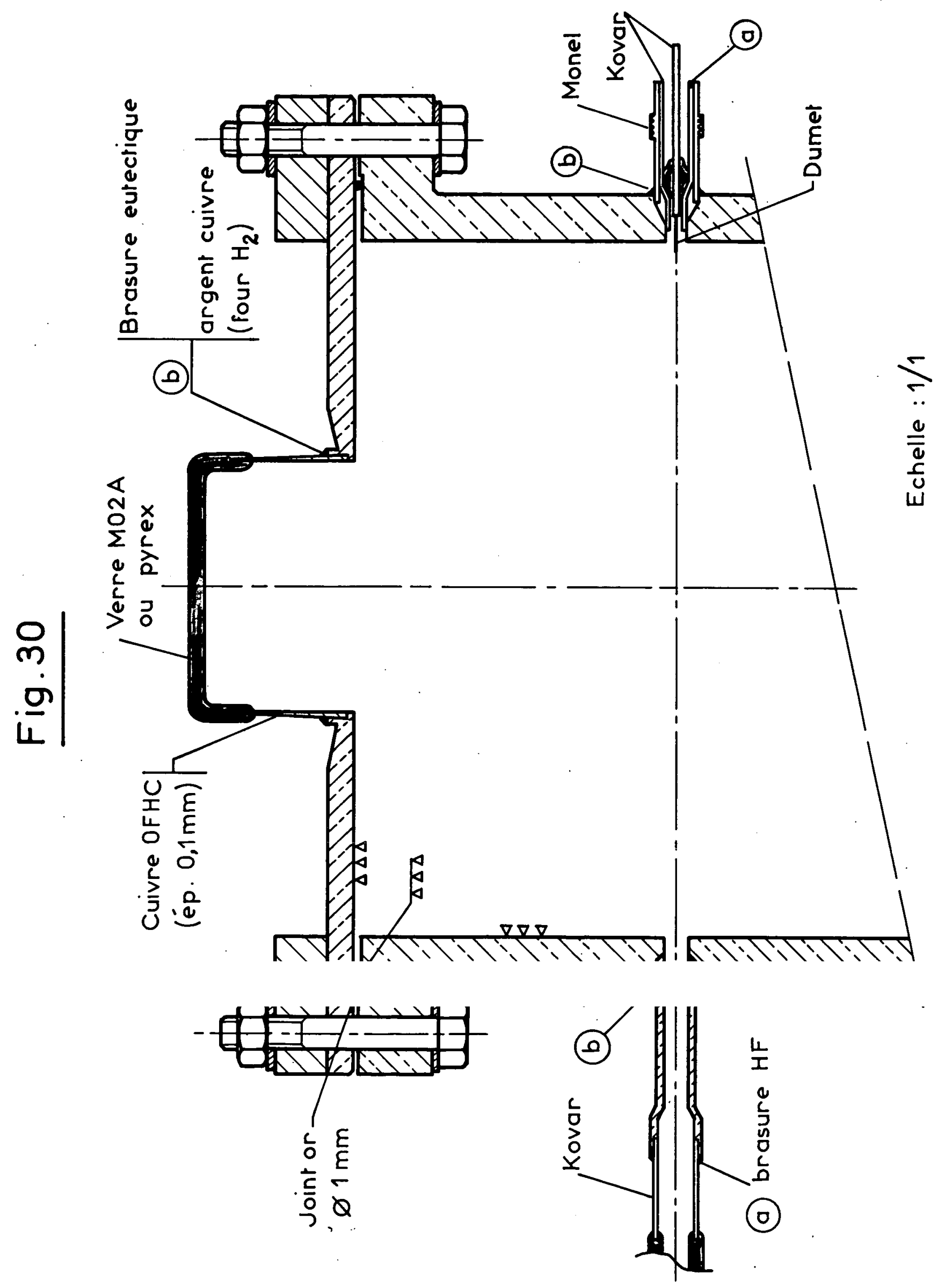




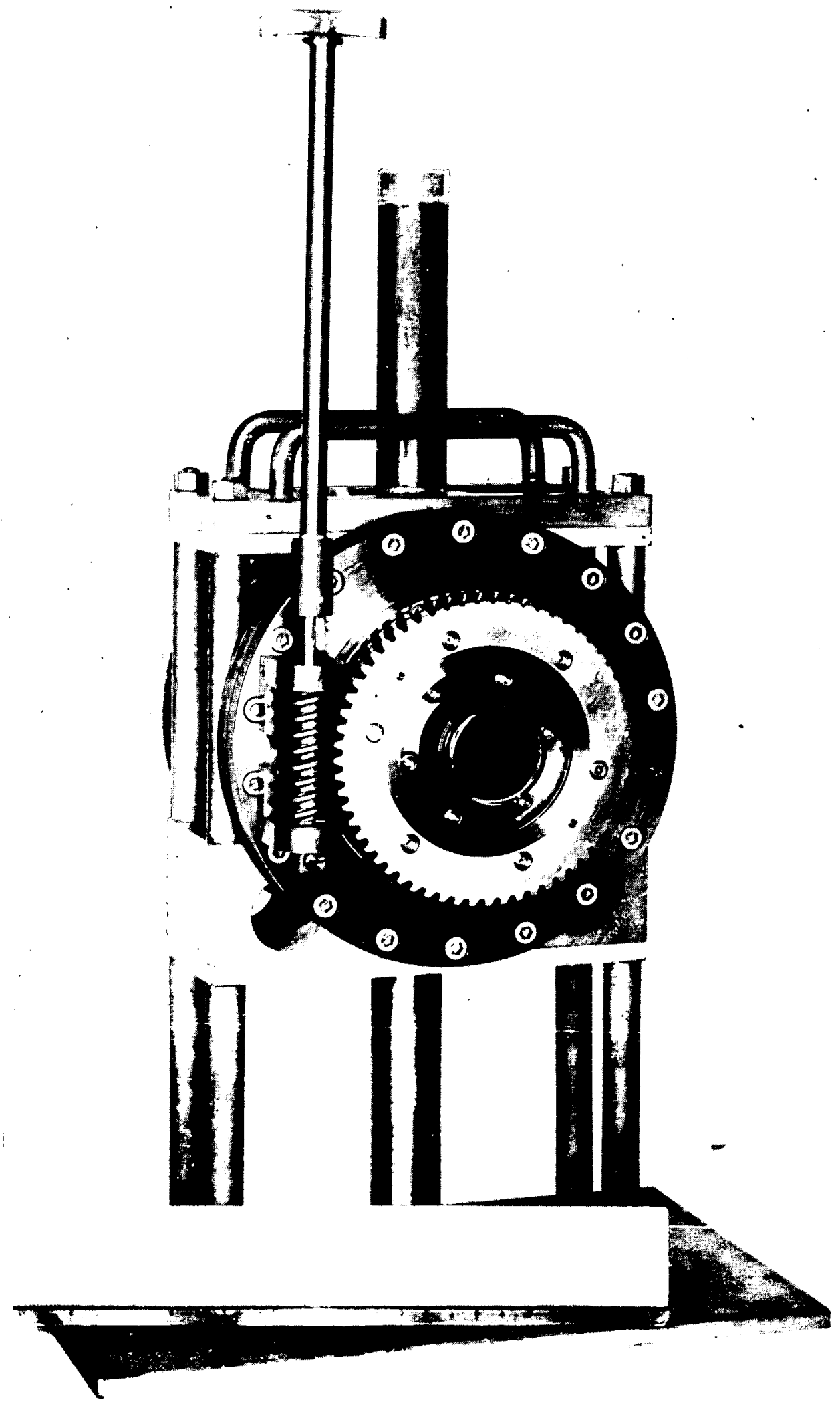


(f) Quelle que soit la méthode retenue pour la réalisation des fonds, il faut encore munir le corps de la cavité de deux queusots et d'une boucle de couplage. Les queusots sont formés d'un raccord pyrex-verre moly-Kovar; la boucle est également montée à l'intérieur d'un petit tube en Kovar. Ces derniers éléments sont rapportés par brasure HF sur des amorces tubulaires en cuivre ou en monel préalablement brasées sous hydrogène sur le corps de la cavité. Cette dernière doit donc, comme nous l'avons dit, être en cuivre OFHC et ce métal, d'importation américaine, n'est disponible qu'en billettes de diamètre trop faible. Il a danc fallu refarger, à partir de celles-ci,des couronnes d'un diamètre suffisant pour permettre l'obtention du corps par usinage ultérieur, ce qui d'ailleurs a l'avantage de donner un métal d'un grain très régulier. L'intérieur est usiné à une tolérance de $\pm 0,015 \mathrm{~mm}$ et subit ensuite un polissage, sans effet notable sur la surtension mais susceptible d'accélérer le dégazage de l'ensemble.

Nous donnons sur la figure 30 le schéma et sur la figure 31 la vue extérieure de la réalisation à laquelle nous avons finalement abouti. Si cette dernière n'a pu encore être obtenue sans présenter aucune fuite, celles-ci ont été toutefois assez faibles pour nous permettre d'utiliser ces cavités pour établir un prévide, comme nous l'avons expliqué ci-dessus : l'une d'elles maintient cette fonction depuis plus d'un an sans que la cellule cylindrique intérieure n'ait encore implosé. (g) Pour éliminer le problème des fuites au niveau des joints, on peut penser à réaliser la cavité en acier inoxydable bas carbone Z3CN18-10 permettant de serrer plus efficacement le joint d'or entre les brides. La mauvaise conductivité électrique de ce métal oblige à cuivrer par électrolyse les surfaces internes; des essais nous ont montré qu'on pouvait obtenir ainsi des surtensions excellentes et, par ailleurs, le problème du dégazage sous vide des dépôts électrolytiques ne semble pas gênant ici. 
Le scellement des fenêtres peut ici encore être effectué par joint Housekeeper $\left({ }^{72}\right)$; l'acier inoxydable ayant la même dilatation que le cuivre $\left(180.10^{-7} /{ }^{\circ} \mathrm{C}\right)$ mais une rigidité mécanique beaucoup plus grande, ces scellements sont encore plus difficiles à réaliser qu'avec le cuivre et seules quelques rares entreprises en assurent la fabrication $\left({ }^{73}\right)$. La mauvaise conductivité thermique de l'acier inoxydable permet, par contre, d'assembler par soudure argan arc les pièces comportant des scellements sans détruire ceux-ci; toutefois, cette opération rendant le métal partiellement ferromagnétique, il peut être préférable de cuivrer l'acier puis d'effectuer des brasures suivant la même technique que pour les cavités en cuivre. Le serrage des joints toriques en or (diamètre $154 \mathrm{~mm}$, épaisseur $1 \mathrm{~mm}$ ) ne doit pas cette fois offrir de difficultés. On peut employer pour cet usage des boulons en acier réfractaire $\times 20 T$ avec des rondelles élastiques de type Schnorr pour rattraper les différences de dilatation; ces pièces étant toutefois magnétiques, il peut être préférable d'utiliser des boulons également en acier inoxydable. Nous mettons actuellement au point un ensemble de cavités de ce type, dont nous avons vu qu'une variante développée indépendamment a permis la réalisation du premier maser autooscillateur à ${ }^{87} R b\left({ }^{3}\right)\left({ }^{4}\right)$. Rappelons que la quantité importante $(50 \mathrm{mg}$ ) de rubidium utilisée lors du remplissage dans cette dernière expérience n'a permis le fonctionnement de l'appareil quependant environ un mois; l'alcalin disparaissait donc rapidement par un processus non déterminé (fuites, ou absorption par les parois qui n'avaient pas été paraffinées). (1) On pourrait enfin penser à réaliser des fenêtres avec des scellements aluminemétal ou quartz-métal. 
Qu'elle soit en cuivre ou en acier inoxydable, la cavité est fermée d'un côté par. un fond souple soutenu au centre, de l'extérieur, au moyen d'un système mécanique contrebalançant l'effet de la pression atmosphérique et permettant l'accord par une légère déformation du fond. Le système de commande, en acier inoxydable, peut être en grande partie démonté lors de l'étuvage : l'emploi du graphite comme lubrifiant supprime d'ailleurs le risque de grippage même après une cuisson à haute température, si toutefois les dimensions des pièces ont été stabilisées par plusieurs chauffages et refroidissements successifs.

Signalons enfin l'intérêt que présente, au cours de l'assemblage, un contrôle fréquent de l'étanchéité au moyen d'un spectrographe de masse à hélium. La haute sensibilité de cet appareil est nécessaire : une fuite de $10^{-10}$ torr $\times \ell / \mathrm{s}$ conduit en effet, au bout d’une journée, à une pression partielle d’oxygène dans la cavité atteignant $10^{-6}$ torr, c'est-à-dire égale à la tension de vapeur saturante du rubidium à $39^{\circ} \mathrm{C}$ 。 
(1) Mc COUBREY (A.0.) - Proceedings of the IEEE, 1966, 54, 116

$\left.{ }^{2}\right) \operatorname{DICKE}\left(\mathrm{R} . \mathrm{H}_{0}\right.$ ) et al - Microwave Atomic Oscillator using Rubidium 87 (8 ${ }^{\text {th }}$ Quarterly Report), Princeton University, 1957

$\left({ }^{3}\right)$ DAVIDOVITS (P.) et STERN (W.A.) - Applied Physics Letters, 1965, ․ 20

$\left({ }^{4}\right)$ DAVIDOVITS (P.) et NOVICK (R.) - Proceedings of the IEEE, 1966, 54, 155

$\left({ }^{5}\right)$ BDUCHIAT (M.A.) - Thèse, Paris 1964; Publications Scient. et Techn. du Min. de I'Air, 1965, NoT. 146

$\left({ }^{6}\right)$ BOUCHIAT (M.A.) et BROSSEL (J.) - Phys. Rev., 1966, 147, 41

(7) HARTMANN (F.) - Journal de Physique, 1967, 28, 288

$\left({ }^{8}\right)$ HARTMANN (F.) - Physics Letters, 1967, 24 A, 767

$\left({ }^{9}\right)$ MASNOU-SEEUWS (F.) et BOUCHIAT (M.A.) - Journal de Physique, 1967, 28, 406

(10) LAMB (W.E.) et FELD (B.T.) - Phys. Rev., 1945, 67, 25

(11) GROSSETÊTE (F.) - Thèse, Paris 1967, non publiée

(12) GROSSETETE (F.) - Journal de Physique, 1964, 25, 383

(13) BLOEMBERGEN $\left(\mathrm{N}_{\mathrm{a}}\right)$ et SHEN $\left(Y_{0} \mathrm{R}_{\mathrm{a}}\right)$ - Phys. Rev., 1964, 133 A, 37; LAMB (W.E.) - Phys. Rev., 1964, 134 A, 1429

(14) MinguZzi (P.), STRUMiA (F.) et VIOLINo (P.) - Nuovo Cimento, 1966, 46 B, 145

(15) BOUCHIAT (M.A.) - Journal de.Physique, 1965, 26, 415

(16) HARTMANN (F.), RAMBOSSON (M.), BROSSEL (J.) et KASTLER (A.) - Comptes Rendus Acad. Sci., 1958, 246, 1522

(17) COHEN-TANNOUDJI (C.) et LALÖ̈ (F.) - Journal de Physique, 1967, 28, 505 et 722 (18) FIRESTER (A.H.) et CARVER (T.R.) - Phys. Rev. Letters, 1966, 17, 947; Phys. Rev., 1967, 164, 76

(19) ARDITI (M.) et CARVER (T.R.) - Phys. Rev.,1964, 136 A, 643

(20) ABRAGAM (A.) - Principles of Nuclear Magnetism, Oxford Press, 1961, Ch. III

(21) SLATER (J.C.) - Rev. Mod. Phys., 1946, 18, 441; Microwave Electronics, Van Nostrand, 1950, P. 78

(22) KLEPPNER (D.), GOLDENBERG (H.M.) et RAMSEY (N.F.) - Phys. Rev., 1962, 126, 603 
(23) VUYLSTEKE (A.A.) - Elements of Maser Theory, Van Nostrand, 1960, ch. 5

(24) AUDOIN (C.) - Comptes Rendus Acad. Sci., 1966, 263 B, 542

(25) GRIVET (P.) et BLAQUIERE (A.) - Proceedings of the Symposium on Optical Masers, New York 1963, p. 69

(26) VESSOT (R.F.C.), MUELLER (L.F.) et VANIER [J.) - Proceedings of the IEEE-NASA Symposium on Short Term Frequency Stability, Greenbelt 1964, p. 111

(27) GRIVET (P.) et BLAQUIERE (A.) - Le Bruit de Fond, Masson, Paris 1958, ch. 5

(28) KOPFERMANN (H.) et KRUGER $\left(\mathrm{H}_{0}\right)$ - Z. Physik, 1936, 103, 485

(29) CH'EN (S.) et TAKEO (M.) - Rev. Mod. Phys., 1957, 29, 20

$\left(3^{0}\right)$ DAVIDOVITS (P.) et KNABLE (N) - Rev. Sci. Instr., 1964, 35, 857

(31) BELL (W.E.) et BLOOM (A.L.) - Phys. Rev., 1958, 109, 219

( ${ }^{32}$ ) BLOOM (A.L.) et CARR (J.B.) - Phys. Rev., 1960, 119, 1946

( ${ }^{33}$ ) ARDITI (M.) et CARVER (T.R.) - Phys. ReV., 1961, 124, 800

$\left({ }^{34}\right)$ MARRUS (R.), MIC COLM (D.) et YELLIN (J.) - Phys. Rev., 1966, 147, 55

$\left({ }^{35}\right.$ ) BEATY (E.C.), BENDER (P.L.) et CHI (A.R.) - Phys. Rev., 1958, 112, 450

( ${ }^{36}$ ) ALLEY (C.0.) - Quantum Electronics, Columbia University Press, New York 1960, p. 146

( ${ }^{37}$ ) DEHMELT (H.G.) - Bull. Am. Phys. Soc,, 1962, ․, 615

$\left({ }^{38}\right.$ ) DAVIDOVITS (P.) et KNABLE (N.) - J. Appl. Phys., 1964, 35, 3042

( ${ }^{39}$ ) BREWER (R.G.) - J. Opt. Soc. Amer., 1962, 52, 832 et Réf. ( ${ }^{5}$ ), p. 113

(40) Tektronix, modèle $502 \mathrm{~A}$

$\left({ }^{41}\right)$ Sylvania, modèle SYD-4302 A

(42) Le pilote et la chaîne de multiplication, réglés à la fréquence désirée, ont été réalisés par la Compagnie Générale de Télégraphie Sans Fil (C.S.F.)

(43) Hewlett-Packard, modèle 2590 A

$\left({ }^{44}\right)$ DRAGONE (C.) - Bell System Tech. J., 1967, 46, 775

( ${ }^{45}$ ) Les écarts de Droitwich par rapport au temps $A_{1}$ sont publiés chaque mois dans l' "Onde Electrique".

$\left({ }^{46}\right.$ ) PENSELIN (S.), MORAN (T.), COHEN (V.W.) et WINKLER (G.) - Phys. Rev., 1962, 127, 524

(47) LEL, modèle SBC $3+$ LR $1297 \operatorname{modifié~}$

( ${ }^{48}$ ) Les différentes techniques à mettre en oeuvre pour mesurer les surtensions, les couplages, etc. sont décrites dans la référence suivante : GINTZTON (E.L.) - Microwave Measurements, Mc Graw Hill, New York 1957, ch. 9

(49) Institut de Radioélectronique de l'Académie des Sciences de l'U.R.S.S., Moscou (Communication privée). 
(50) AYMAR-FEHRENBACH (M.) - Thèse 3e cycle, Paris 1967, non publiée, et réf. $\left({ }^{5}\right), p \cdot 107$

(51) Les différentes déterminations de la tension de vapeur saturante du rubidium ne sont pas toujours concordantes. Nous avons utilisé celle de la référence suivante, qui semble la meilleure : HONIG (R.E.) - R.C.A. Review, 1957, 18, 195

(52) Réf. $\left({ }^{5}\right), p \cdot 98$

(53) ROBINSON (L.B.) - Phys. Rev., 1960, 117, 1275

(54) BENDER (P.L.), BEATY (E....) et CHI (A.R.) - Phys. Rev. Letters, 1958, 1, 311

$\left({ }^{5}\right)$ DAVIDOVITS (P.) - Appl. Phys. Lett., 1964, $\underline{5}, 15$

(56) ROBERTSON (J.) - Electronic News, 24 Juillet 1967

( ${ }^{57}$ ) ANGOT (A.) - Compléments de Mathématiques, Ed. de la Revue d’Optique, Paris 1965, P. 403

(58) WILSON (I.G.), SCHRAMM (C.W.) et KINZER (J.P.) - Bell system Technical Journal, $1946,25,408$;

KINZER (J.P.) et WILSON (I.G.) - Bell System Technical Journal, 1947, 26, 31. Ces deux articles sont reproduits dans l'ouvrage suivant : Radar Systems and Components, Van Nostrand, New York 1949, p. 909 et 936

(59) ANGOT (A.) - op. cit., p. 466;

BORGNIS (F.) - Ann. Phys. Lpz., 1939, 35, 359

$(60)$ ANGOT (A.) - op. cit., p. 371

(61) WITTKE (J.P.) et DICKE (R.H.) - Phys. Rev.,1956, 103, 620

(62) Heraeus GmbH, Hanau, Allemagne

(63) ANGLADE (M.J.) - Le Vide, 1964, 109, 665

(64) HAIT (P.W.) - VacuUm, 1967, 17, 547

(65) KENDALL (B.R.F.) et ZABIELSKI (M.F.) - J. Vac. Sci., 1966, 3, 114;

BROWN (R.D.) - VacuUm, 1967, 17, 505

(66) SAYERS (J.F.) - J. Sci. Instr., 1960; 37, 203

(67) MARTIN (G.) - Rev. Sci. Inst., 1963, 34, 707;

VOGL (T.P.), MC INTOSH (R.0.) et GARBUNY (M.) - ReV. Sci. Inst., 1965, 36, 1439;

EISENSTADT (M.M.) - Rev. Sci. Inst., 1967, 38, 134

(68) HOLDEN (J.), HOLLAND (L。) et LAURENSON (L.) - J. Sci. Inst., 1959, 36, 281

(69) REIMANN (A.L.) - Technique du Vide, Dunod,fai is 1358, ch. 10

(70) FISCHHOFF (E.), PREVOT (F.) et SLEDZIEWSKI (Z.) - Le Vide, 1962, 99, 195;

SOUCHET (R.) - Le Vide, 1966, 123, 235

( ${ }^{71}$ ) LEA-WILSON (P.) et BAKER (A.) - S.E.R.L. Technical Journal, 1956, 6, 99

(72) BENBENEK (J.E.) et HONIG (R.E.) - Rev. Sci. Inst,, 1960, 31, 460;

COLGATE (S.0.) et WHITEHEAD (E.C.) - Rev. Sci. Inst., 196 $\overline{2}, 33,1122$

( ${ }^{73}$ ) Larson Electronic Glass Co, Redwood City, California, U.S.A. 